\title{
MOLECULAR MECHANISMS OF AMPA AND KAINATE RECEPTOR GATING AND ITS IMPLICATION IN SYNAPTIC TRANSMISSION
}

\author{
Dissertation \\ Zur Erlangung des Doktorgrades \\ der Mathematisch-Naturwissenschaftlichen Fakultäten \\ der Georg-August-Universität zu Göttingen
}

\author{
vorgelegt von \\ Naveen Nagarajan \\ aus Mysore, Indien
}

Göttingen, den 17.9.2002 
D7

Referent:

Prof. Dr. Axel Zeeck

Korreferent:

Prof. Jürgen Troe

Tag der mündlichen Prüfung: 


\section{TABLE OF CONTENTS}

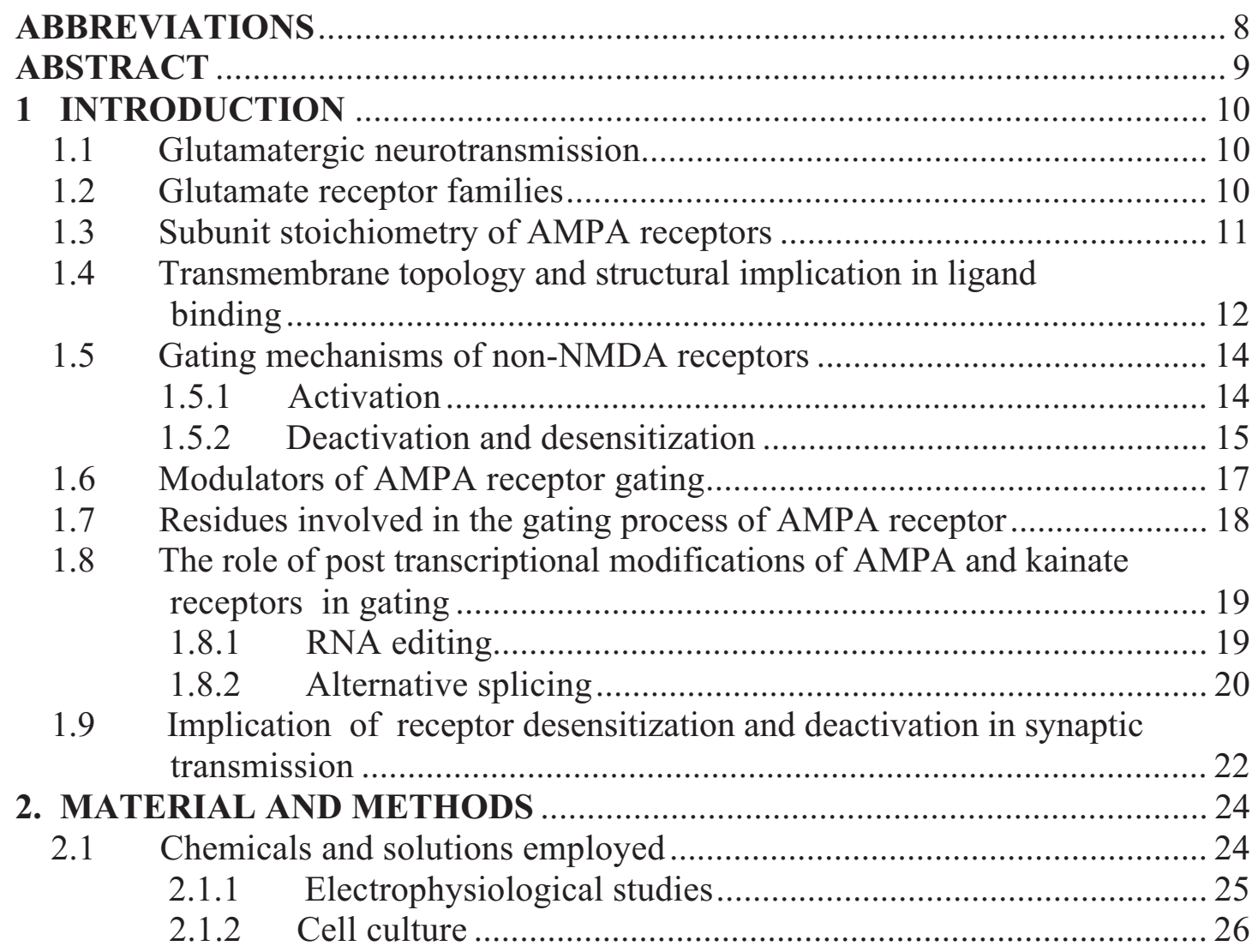

2.2 Preparation of solutions and procedures employed in cell culture and

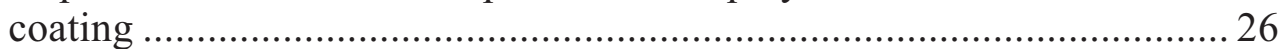

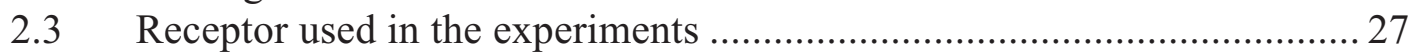

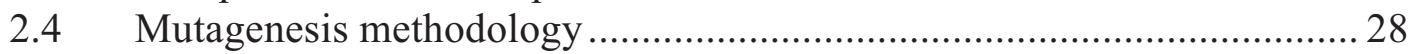

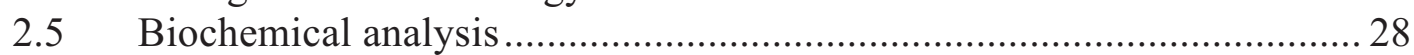

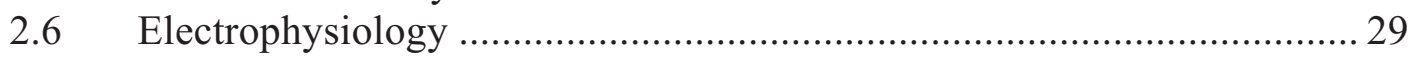

2.6.1 The equivalent circuit for a simple cell................................... 29

2.6.2 An overview of Patch Clamp technique .................................... 31

2.6.3 Configurations of patch clamp technique .................................. 33

2.6.4 Experimental procedures.......................................................... 35

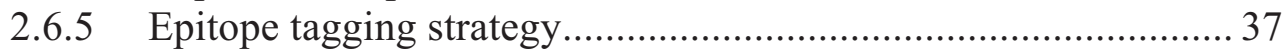

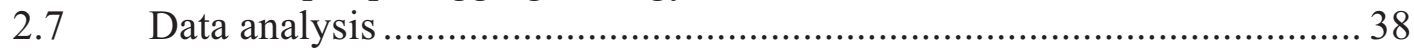

2.7.1 Software used for the data analysis........................................... 38

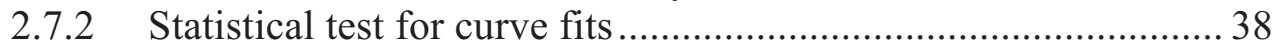

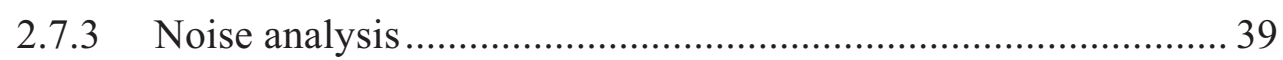

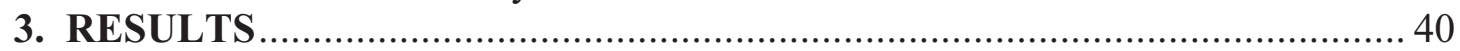

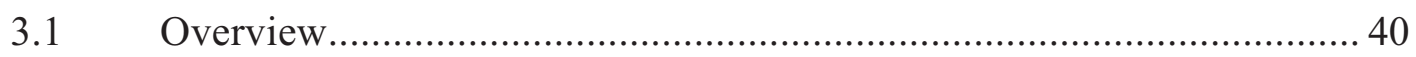

3.2 Gating and assembly properties of AMPA receptors ........................... 40 
3.2.1 Specific gating mechanisms 40

3.3 Nondesensitizing phenotype penetrates more readily than desensitzing phenotype.

3.4 Nonrectifying phenotype penetrates more readily than rectifying phenotype.....

3.5 Plausible mechanisms for homo and heteromeric AMPA receptor assembly

3.6 Protein expression is linear to the transfected cDNA concentration............ 47

3.7 Quantification of functional receptor expression.

3.8 Quantification of single channel properties of homo and heteromeric

AMPA receptors. 50

3.9 Analytical procedures for classification of specific phenotypes in cotransfection experiment.

3.9.1 Quantification of desensitizing and nondesensitizing population of receptors

3.9.2 No evidence for intermediate desensitization phenotype in cotransfection experiments.

3.9.3 Quantification of rectifying and nonrectifying population of receptors in cotranfection experiments

3.9.4 No evidence of intermediate phenotype for rectification properties in cotransfection experiments .....

3.10 A quantitative model of subunit assembly and phenotype dominance ........ 59

3.10.1 Binomial model for homomeric subunit assembly ........................5 59

3.10.2 Selective assembly model for heteromeric receptors .................... 61

3.10.3 Model assumptions ............................................................... 62

3.10.4 Construction of phenotype expression models based on single channel conductance.

3.10.5 Desensitization and Rectification properties in Homo and Heteromeric assembly of AMPA receptors 65

3.10.5.1 Assembly of homomeric receptors...................................65

3.10.5.2 Assembly of heteromeric receptors....................................66

3.10.6 More evidences and consistent results in AMPA receptor assembly by altering the markers on different subunits .................. 68

3.10.6.1 Results on homomeric assembly of subunits..................68

3.10.6.2 Results on heteromeric assembly of subunits................69

3.10.7 Channel properties of AMPA receptor are controlled by spatial arrangement of subunits ............................................................ 70

3.10.7.1 Control by desensitization............................................ 70

3.10.7.2 Control by rectification............................................... 71

3.10 .8 Heterodimerization of AMPA receptors ..................................... 71

3.10.9 Subunit configuration for heterodimeric assembly of AMPA receptors

3.10.10 Possible mechanisms for subunit assembly of AMPA

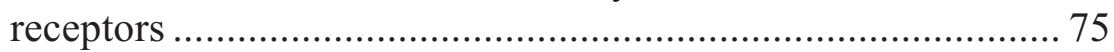

3.10.10.1 Symmetry controlled mechanism...............................75

3.10.10.2 Association controlled mechanism................................77

3.11 Effect of modulators on AMPA receptors .......................................... 78

3.11.1 Effect of modulators on recombinant AMPA receptors ................ 78

3.11.1.1 Effect of CX546 on recombinant GluR1 and GluR2 subunits. 
3.11.1.2 Effect of CX516 and IDRA-21 on recombinant

GluR1 and GluR2 receptors.

3.11.2 Effect of modulators in the absence of desensitization.............. 82

3.11.2.1 Effect of CX516 and IDRA 21 on non-desensitizing GluR1receptors.

3.11.2.2 Effect of CX546 on nondesensitizing GluR1 receptors.

3.11.2.3 Additional evidences for the effect of CX546 on gating properties of nondesensitizing

GluR1receptors

3.11.3 Specific binding properties of CX546

3.11.3.1 Determining the binding properties of CX546 to AMPA receptors..

3.11.3.1.1 Kinetics of binding in the presence of saturating concentration of glutamate.

3.11.3.1.2 Kinetics of binding in the presence of intermediate concentration of glutamate.

3.11.3.1.3 Analysis of the receptor kinetics for potentiation and recovery mediated by CX546.....

3.11.3.2 Comparison of the binding properties of CX546 with other AMPA receptor modulators.

3.11.4 Effect of modulators on native receptors ....

3.11.5 Effect of modulators on synaptic transmission ........................ 94

3.11.6 Mimicking the gating properties of synaptic receptors by deactivation kinetics.

3.12 Studies on the splice variants of kainate receptors

3.12.1 Role of C-terminus in controlling the gating and expression properties of GluR6 subunit

3.12.2 Characterization of gating properties of splice variants of GluR5 subunit.

3.12.2.1 Characterization of homomeric splice variants of GluR5 isoforms.

3.12.2.1.1 The isoforms of GluR5 splice variants and their N-terminal deletion are functional.

3.12.2.1.2 Effect of $\mathrm{N}$-terminal deletion on gating properties of GluR5 isoforms. 104

3.12.2.1.2.1 Effect on the rise time and time constant of desensitization. .104

3.12.2.1.2.2 Effect on the amount of desensitization. 106

4. DISCUSSION

4.1 Mechanism of Heteromeric assembly of AMPA receptors and their functional implications.

4.1.1 Model assumptions . 108

4.1.2 Theoretical, Biochemical and Structural consequences in assembly of AMPA receptors 
4.1.3 Possible mechanisms for AMPA receptor assembly

4.1.4 Functional significance of selective assembly in AMPA receptors

4.2 Probing mechanism of AMPA receptor desensitization with chemically defined AMPA receptor modulators

4.2.1 Distinguishing action of CX546 over other AMPA

modulators on AMPA receptors.

4.2.2 Mechanism of the action of CX546....

4.2.3 Physiological and pharmacological implications of the action of CX546 on AMPA receptors

4.3 Biophysical properties and the role of kainate receptors in synaptic transmission

4.3.1 Physiological role of C-terminus region of GluR6 subunit

4.3.2 Potential physiological functions of the extracellular and intracellular domains in GluR5 subunit

4.4 FUTURE DIRECTIONS 


\section{ABBREVIATIONS}

\begin{tabular}{|c|c|}
\hline AMPA & $\alpha$-amino-3-hydroxy-5-methyl-4-isoxazolepropionate \\
\hline BES & (N,N-bis[2-Hydroxyethyl]-2-aminoethanesulphonic acid) \\
\hline CNS & Central nervous system \\
\hline CTZ & Cyclothiazide \\
\hline CX516 & 1-(Quinoxalin-6-ylcarbonyl) piperidine \\
\hline CX546 & 1-(1,4-Benzodioxan-6-ylcarbonyl)piperidine \\
\hline EGTA & $\begin{array}{l}\text { Ethylene glycol-bis ( } \beta \text { amino ethyl ether)-N,N, N',N'- } \\
\text { tetraacetic acid }\end{array}$ \\
\hline EPSC & Excitatory post synaptic current \\
\hline GluR & Glutamate receptor \\
\hline GABA & $\gamma$-aminobutyric acid \\
\hline GFP & Green fluorescent protein \\
\hline HEPES & $\begin{array}{l}\text { (N-[2 hydroxy ethyl] piperidine-N'-[2-ethane sulfonic } \\
\text { acid]) }\end{array}$ \\
\hline IDRA 21 & $\begin{array}{l}\text { 7-Chloro-3-methyl-3,4-dihydro- } 2 H \text {-1,2,4-benzothiadiazine } \\
S, S \text {-dioxide }\end{array}$ \\
\hline iGluR & Ionotropic glutamate receptor \\
\hline PSD & Postsynaptic density \\
\hline NMDA & N-methyl-D-aspartate \\
\hline $\mathrm{Na}_{2} \mathrm{HPO}_{4} \cdot 12 \mathrm{H} 2 \mathrm{O}$ & Di-sodium hydrogen phosphate \\
\hline TTX & Tetrodotoxin \\
\hline
\end{tabular}




\section{ABSTRACT}

In this work we have studied the biophysical properties of AMPA and kainate receptors, which are found mainly in central synapses. AMPA receptors are thought to be tetrameric in nature. The permeability and gating of AMPA-receptors are controlled by the heteromeric assembly of GluR2 with GluR1/3/4 subunits. To investigate the underlying molecular mechanism, responses from homomeric or heteromeric GluR1/2 receptors were quantified using patch clamp technique by excising outside out patches from transfected HEK cells in conjugation with fast flow application system. The subunits carried markers for controlling rectification and desensitization. We quantitatively monitored both properties as a function of subunit composition and relative expression level. We have found that GluR1/GluR2 receptor subunits do not assemble randomly, but assemble in preferential fashion as heterodimers with two identical subunits positioned on opposite sides of the tetramer. Incorporation of these data in an assembly model indicated that GluR1 binds to GluR2 4-6 times more rapidly during assembly than with GluR1.

The second part of the work was to test the effect of two classes of modulators, the benzothiadiazides (cyclothiazide and IDRA 21) and benzoylpiperidines (CX516 and CX546) on the gating kinetics of the AMPA receptor. We found that CX546 reduced the degree of desensitization more potently than CX516 or IDRA 21. The desensitization of GluR2 receptors was inhibited more than that for GluR1 in the presence of CX516/CX546. Analysis of modulator action revealed that CX546 binds specifically to the agonist bound nondesensitized state of the receptor and acts by destabilizing the desensitized conformation of the receptor. The unique properties of CX546 different from the action of cyclothiazide may be beneficial for therapeutical applications.

The third part of the work concentrates on characterizing the kinetic properties of kainate receptors. Biophysical studies (of splice variants) were made to understand how the C-terminal deletion of GluR6 subunit affects the gating kinetics. In addition to this we studied the functional and gating properties of various splice variants in GluR5 subunit. We found out the importance of extra 15 amino acids cassette in the N-terminus of GluR5 subunit (GluR5A and GluR5B isoforms) in controlling the 
gating properties of the receptor.

\section{INTRODUCTION}

\subsection{Glutamatergic neurotransmission}

Fast excitatory neurotransmission in the mammalian central nervous system is mainly mediated by the neurotransmitter glutamate. Similar to the transmitter acetylcholine at neuromuscular junction, glutamate at central synapses are important in transducing chemical signal to electrical ones. Communication between nerve cell takes place at specialized regions termed as synapse. An average neuron forms several thousands of synaptic connections and receives as many as 100,000 input connections. The strength of the synaptic connections can be either enhanced or diminished by different cellular activity. These changes are thought to be cellular basis for the memory formation.

An individual synapse of a nerve cell is made up of pre and postsynaptic zones separated by the synaptic cleft. Upon arrival of the action potential, presynaptic neuron initiates the release of glutamate that diffuses across the synaptic cleft to interact with glutamate receptors on the membrane of postsynaptic cell.

Specific alterations of glutamatergic neurotransmission have been related to the neuronal damage observed after episodes of ischemia and hypoglycemia, as well as to the etiology of a series of neurological conditions in epilepsy, Alzheimer's disease, amyotrophic lateral sclerosis, Parkinson's and Huntington's disease, schizophrenia, epilepsy, Rasmussen's encephalitis and stroke (Jonas P and Monyer H., 1999). Hence a clear understanding of the structural and functional properties of glutamate receptors becomes essential.

\subsection{Glutamate receptor families}

The family of glutamate receptors (GluRs) are categorized into two distinct classes, ionotropic and metabotropic receptors (Hollmann and Heinemann, 1994). The ionotropic glutamate receptors (iGluRs) comprise cation-specific ion channels. Molecular cloning and pharmacological approaches have classified ionotropic glutamate receptors into three groups: $\alpha$-amino-3-hydroxy-5-methyl-4isoxazolepropionate (AMPA), kainate and N-methyl-D-aspartate (NMDA) receptor channels. 
Glutamate receptors of the AMPA subtype are expressed mainly in central nervous system. On the basis of sequence homology and characteristics of the expressed receptors the subunits of AMPA receptors are classified into four individual subunits (GluR1-4), which share 68-73\% sequence identity (Dingledine et al., 1999). The amount and pattern of expression vary with the embryonic stages (Dingledine et al., 1999).

The kainate receptor family is composed of GluR5-7, KA-1, KA-2 subunits. As compared to AMPA receptor, the expression patterns of the different subunits of kainate receptor family are heterogeneous (Bahn et al., 1994; Wisden and Seeburg, 1993). GluR5 transcripts are found mainly in DRG neurons,subiculum, septal nuclei, periform and cingulate cortices and in the purkinje cells of the cerebellum. GluR6 transcripts are most abundant in the dentate gyrus and the CA3 region of the hippocampus and in the striatum.

\subsection{Subunit stoichiometry of AMPA receptors}

AMPA channels are constructed as heterooligomers comprising GluR1-4 subunits. AMPA receptors are thought to be tetrameric in nature (Rosenmund et al., 1998) similar to voltage-gated potassium channels, cyclic nucleotide-gated channels (Liu et al., 1998) and NMDA receptors (Laube et al., 1998). This was determined by electrophysiological and pharmacological approaches (Rosenmund et al., 1998), although a few line of evidences are in favor of pentameric subunit stoichiometry (Ferrer-Montiel and Montal, 1996). The stoichiometry of AMPA receptor may be fixed similar to other class of receptors like $\mathrm{GABA}_{\mathrm{A}}$ receptors (Tretter et al., 1997)or glycine receptors [Kuhse, $1993 \# 331$.

In neuronal population multiple subtypes of AMPA receptor exists based on the subunit composition. The subunit composition in several brain regions may have several preferential combinations of subunits that are required for dual functions of physiological relevance. As for example, it was found that the calcium permeability in retinal ganglion neurons (Zhang et al., 1995) and polyamine sensitivity in hippocampal interneurons (Washburn et al., 1997) differs significantly due to variable subunit composition. On the other side, in auditory nerve synapses, AMPA receptors are composed of GluR4 subunits through which the rapid repetitive synaptic transmission necessary for hearing occurs.

In the first part of the thesis work we studied how subunits assemble and 
particularly examined whether two coexpressed subunits (GluR1/2) combine at random to form channels, or preferentially assemble with a specific stoichiometry and spatial configuration. We show that homomeric receptors assemble in stochastic fashion while heteromeric receptors assemble in preferential manner with a stoichiometry of two GluR1 and two GluR2 subunits. Furthermore, we show that identical subunits should be positioned on opposite sides of the channel pore for optimal function of the AMPA channel.

\subsection{Transmembrane topology and structural implication in ligand binding}

Ionotropic glutamate receptors are modular proteins having extra and intracellular domains. The four distinct transmembrane domains comprising the channel contain M1, M3, M4 and a pore forming loop (M2-loop) (Paas, 1998; Wo and Oswald, 1995) similar to potassium channel. The polar residues within the M2-loop of channel pore region control the rapid ion permeation and ion selectivity by a number of factors including electrostatic coupling, hydration and pore dimension. These residues also play an important role in determining single channel conductance both in non-NMDA and in NMDA receptors (Behe et al., 1995; Kupper et al., 1996; Premkumar et al., 1997). This is shown in figure1.

The extracellular amino-terminal domain of eukaryotic ionotropic glutamate receptors show low-level sequence identity to the bacterial leucine/isoleucine/valine binding protein (O'Hara et al., 1993). The first $\sim 400$ amino acids are homologous to the lysine/isoleucine/valine-binding protein (LIVBP). The next $\sim 150$ amino acids preceding M1 and the segment between M3 and M4 are homologous to both the glutamine-binding protein (QBP) and the lysine/arginine/ornithine-binding protein (LAOBP). The extracellular amino terminal domain is not involved in the ligand binding (Stern-Bach et al., 1994) but is important for subtype-specific assembly (Leuschner and Hoch, 1999) and mediates intersubunit interactions (Ayalon and Stern-Bach, 2001; Kuusinen et al., 1999). Furthermore, receptor anchoring and signal transduction occurs by the interaction of carboxy terminus region with postsynaptic density proteins (Sheng and Pak, 2000). 


\section{TRANSMEMBRANE TOPOLOGY OF AMPA RECEPTORS}

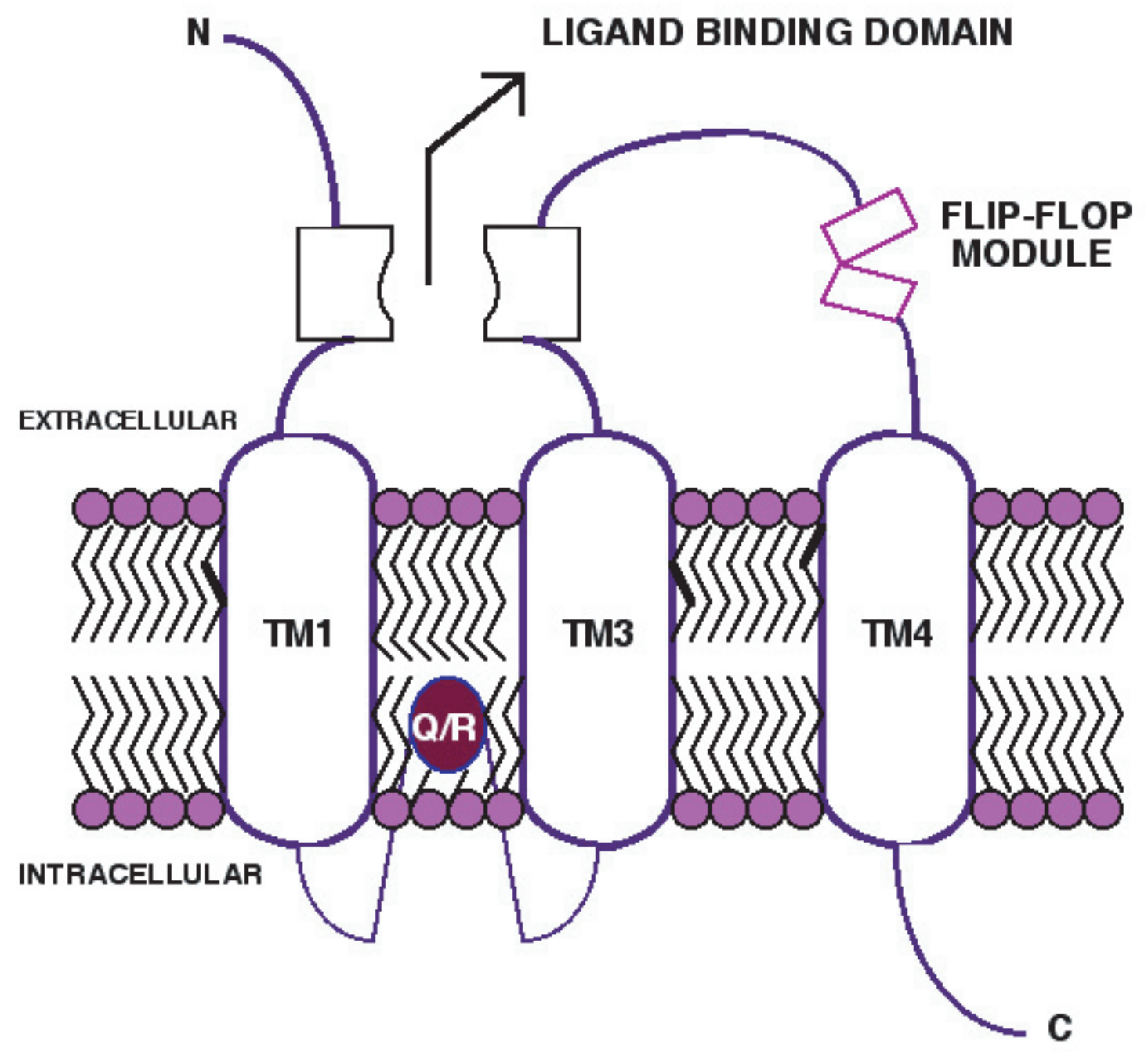

Figure 1. Transmembrane topology of AMPA receptors

The figure describes the transmembrane domains TM1, TM3 and TM4. The non -transmembrane domain forms the channel pore and carries either a glutamine (Q) or arginine $(\mathrm{R})$ residue in the channel pore region. The $\mathrm{N}$-terminus is located in the extracellular region while $\mathrm{C}$ terminus is present in the intracellular region. The ligandbinding pocket is made up of residues from S1 and S2 globular domains (as shown by the boxes on TM1 and TM3 correspond). Flip/Flop module represents the region for alternative splicing.

The ligand-binding core is subdivided into two halves of polypeptide segments based on site-directed mutagenesis and chimeric polypeptide fusion experiments (Paas, 1998). The first half (S1 region) includes nearly 150 amino acids aminoterminal to first transmembrane segment while the second half ( $\mathrm{S} 2$ region) covers a portion between transmembrane segments M3 and M4. 
The domain S1 and S2 also possess the residues that make ion pair and hydrogen bonding interactions with the carboxyl and amino groups of AMPA agonists (Armstrong and Gouaux, 2000). A point mutation from lysine 449 of domain 1 in GluR1 to glutamine residue is sufficient to increase the affinity for glutamate ( $\mathrm{Li}$ et al., 1995). The activity of AMPA binding could be abolished by mutating highly conserved arginine residue (Arg 485) to lysine in GluR1 subunit (Kawamoto et al., 1997a) or GluR4 S1S2 (Lampinen et al., 1998) region. The other important features of S1S2 ligand-binding core includes the recently identified zinc binding sites pinpointing the conserved histidine residues in these region as important candidate for zinc binding (Armstrong and Gouaux, 2000).

\subsection{Gating mechanisms of non-NMDA receptors}

The biophysical properties of AMPA receptors are characterized by fast kinetics of activation, deactivation, desensitization and resensitization that occurs on a time scale of few milliseconds to seconds. The conformation leading to the opening and closing of channel is termed as gating phenomenon(Jonas and Sakmann, 1992). We describe each of these processes in detail.

\subsubsection{Activation}

Using electrophysiological and pharmacological approach (Rosenmund et al., 1998) it was shown that AMPA receptor passes through three distinguishable conformational states (small, medium and large) each with a different mean conductance upon progressive binding of glutamate molecules to the receptor.

\section{Four step model of receptor activation}

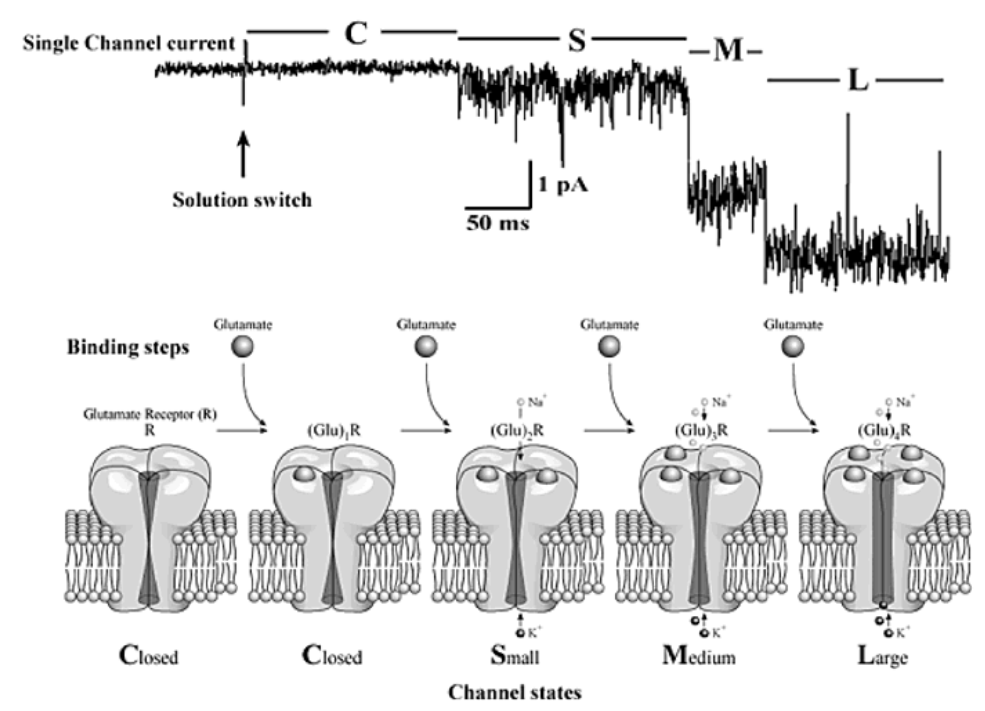




\section{Courtesy from C. Rosenmund}

\section{Figure 2. Four step model of receptor activation}

The upper trace corresponds to single channel recording using patch clamp technique where a single GluR6/GluR3 channel switches to different conformational states upon binding of one, two, three and four glutamate molecules. Upon binding of successive molecules of glutamate, the channel enters into small (two glutamate molecules bound), medium (three glutamate molecules bound) and large (four glutamate molecules bound) conducting states.

The fast activation is measured by the fast rise time $(0.2-0.4 \mathrm{~ms})$ which approaches the resolution of the rapid application technique.

\subsubsection{Deactivation and desensitization}

Following activation by a brief (1-2 ms) exposure to glutamate, the current decays with a characteristic time course known as "deactivation". Prolonged exposure of glutamate results in a long lasting shut state ("desensitized state") of the receptor. In desensitized state, the receptor remains bound to glutamate. This is demonstrated in glutamate receptor gating model.

\section{Glutamate receptor gating model}

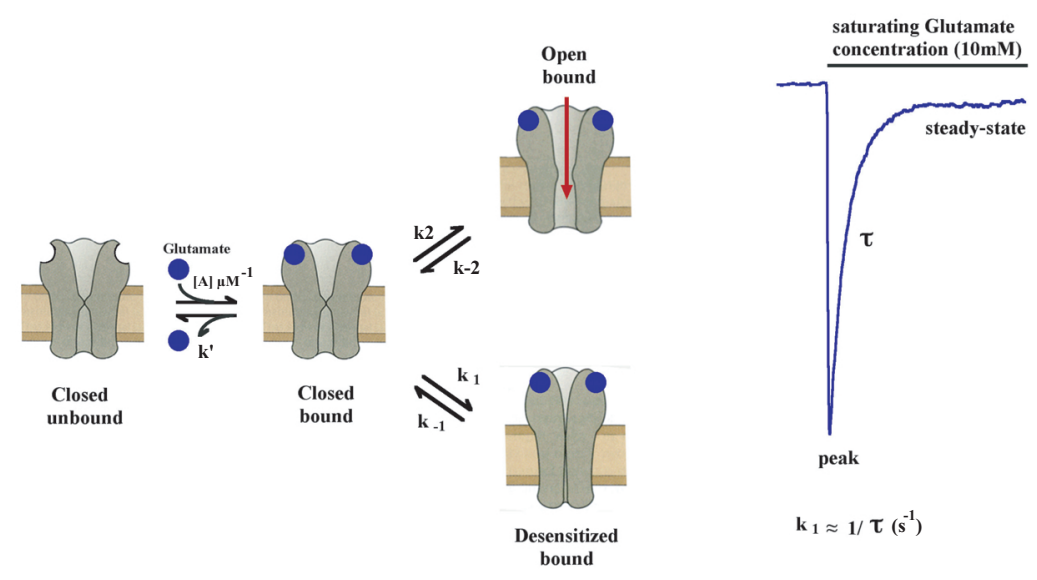

\section{Figure 3. Gating model for glutamate receptor}

The model describes up to four different conformational states of the receptor. 
Binding of the glutamate (blue ball) leads to closed-bound state of the receptor, which then either enters into open-bound or bound-desensitized state. Red arrow depicts the influx of ions in the open bound state of the receptor. The trace on the right side (blue trace) represents an electrical response obtained by using patch-clamp technique to demonstrate gating kinetics involving the activation and desensitization phenomena upon application of saturating concentration of glutamate $(10 \mathrm{mM})$. [A] represents the concentration of agonist, k' represents the rate of receptor entry from closed-bound to unbound state, $\mathrm{k}_{1}$ the rate of receptor entry into desensitized state, $\mathrm{k}_{-1}$ represents the rate of receptor out of the desensitized state. $\mathrm{k}_{2}$ and $\mathrm{k}_{-2}$ represent the rate of transition between the bound and open state. The transition rate from unbound to bound state is product of the rate constant and concentration of agonist. The rate of desensitization $\left(1 / \tau\left(s^{-1}\right)\right)$ can be determined by inverse of the time constant obtained by exponential fit to the desensitizing phase. The amount of desensitization is measured by dividing the peak current amplitude with the steady state amplitude (Peak/Steady state).

In in-vitro biophysical studies of ion channels, the intrinsic channel kinetics are often studied using a rapid switch to a saturating concentration of agonist which minimizes the heterogeneity in the receptor population that are in bound and unbound conformational states. The sensitivity of patch clamp technique make it possible to detect the distinct conformational states that account for a miniscule fraction of total receptor. It also provide valuable clues regarding the involvement of these conformational states during transition process. The kinetic behavior of the channel can be understood by statistical means using simple Markov models which involve microscopic transition rates and state occupancies as important parameters in determining the gating kinetics. Kinetic modeling (Jones and Westbrook, 1996) suggests that the rate of receptors entry into desensitized state is much faster than its exit from the desensitized state. These kinetic parameters are helpful to understand how different conformational states of the channel contribute to the integrated postsynaptic signalling at the synapse.

Activation of AMPA receptors is coupled to rapid desensitization which is accompanied by a $\sim 5$ - to 10-fold increase in glutamate affinity (as determined by the effects of allosteric modulators on ligand binding) (Kessler et al., 1996). Thus, activation and desensitization although occur in a structural and physiologically distinct fashion, they are kinetically interlinked conformational transitions of the agonist-bound state of the receptor. The energetic difference between the activated and desensitized states has been estimated to be in the order of only 1-2 hydrogen bonds based on the estimation of affinity differences between nondesensitized and desensitized states of the receptor (Armstrong and Gouaux, 2000). 
Unlike AMPA receptors, the biophysical properties and the functional relevance of kainate receptors in different brain areas are less understood. The whole cell current responses mediated by recombinant kainate receptors exhibit diverse physiological and pharmacological properties depending upon the subunit composition (Chittajallu et al., 1999).

As a part of the thesis work, we studied the gating properties of AMPA and kainate receptors using different configurations of patch clamp technique. We show the characterization of the basic gating properties like activation, desensitization, amount of desensitization and resensitization kinetics of AMPA and kainate receptors.

\subsection{Modulators of AMPA receptor gating}

The gating kinetics of AMPA receptors can be modulated by variety of compounds like aniracetam, diazoxide, cyclothiazide, PEPA, thiocyanate. These modulators act via different molecular mechanisms to reduce desensitization (Dingledine et al., 1999). The important class of modulators include benzothiadiazines such as cyclothiazide, diazoxide and IDRA21 (Bertolino et al., 1993; Patneau et al., 1993; Yamada and Rothman, 1992; Yamada and Tang, 1993), the pyrrolidinone analogues aniracetam, piracetam (Gouliaev and Senning, 1994; Isaacson and Nicoll, 1991; Partin et al., 1996; Tang et al., 1991) and a heterologous group of substances named ampakines after their property to modify AMPA receptor function. Ampakines include modulators such as BCP, CX516, CX546 (Arai et al., 1996; Holst et al., 1998; Lauterborn et al., 2000; Lynch, 1998). Most modulators are relatively specific for AMPA-type glutamate receptors. Cyclothiazide is the best understood and the most potent modulator of desensitization among benzothiadiazine (at least on flip splice variants) (Dingledine et al., 1999).

Ampakines are molecular entities that have shown their importance as positive modulators in enhancing memory of rodents and humans (Granger et al., 1996; Ingvar et al., 1997). The potential significance of ampakines in their therapeutic effectiveness arise due to their ability to freely cross the blood-brain barrier, (Staubli et al., 1994a) promote induction of long term potentiation in vivo, (Lynch, 1998) improve short term memory in radial maze (Staubli et al., 1994a), facilitate olfactory learning (Larson et al., 1995), and effective boosting of transmission in complex neuronal networks (Sirvio et al., 1996). 
In the second part of the thesis work, investigation were made to understand the pharmacological action of structurally distinct modulators IDRA21 (a benzothiadiazine derivative) and the ampakines CX516 and CX546 (benzoylpiperidine derivatives) on flip version of GluR1/2 subunits, native receptors and on synaptic transmission. The basic kinetic properties of the modulatory action of CX546 have been characterized to gain insight about the molecular mechanism during channel gating and desensitization process. One functional importance is that, IDRA21, CX516, cyclothiazide and CX546 modulates the excitatory synaptic currents by moderately enhancing the peak amplitude and slowing down of evoked postsynaptic currents (EPSCs), although by different efficacy.

\subsection{Residues involved in the gating process of AMPA receptor}

Gating modes can be altered and manipulated within the receptor by several ways to adopt a conformation that have distinct electrophysiological characteristics. Ligand binding activity can be abolished by mutating a conserved charged residue (Arg 485) in either GluR1(Kawamoto et al., 1997b) or GluR4-S1S2 subunit (Lampinen et al., 1998). Leucine residue at the position 650 in GluR1 subunit when mutated to threonine will have 6-20-fold reduction in $\mathrm{EC}_{50}$ value for kainate as compared to wild type receptors $\left(\mathrm{EC}_{50}\right.$ value is used to measure the potency of a drug. It is the effective concentration of drug that causes $50 \%$ of maximum response). The mutant L650T also exhibited approximately two-fold greater potentiation by cyclothiazide as compared to wild-type receptors implying an increase in the extent of desensitization (Armstrong and Gouaux, 2000). This hydrophobic residue distinguishes the binding clefts for the domain closure mediated either by a full agonist (glutamate) or a partial agonist (kainate).

An exchange of highly conserved single hydrophobic residue leucine of short $\alpha$-helix (residing on the surface away from the center) by point mutation to aromatic bulky residue tyrosine is necessary and sufficient for converting the desensitizing receptors to a non-desensitizing phenotype in AMPA receptors (Stern-Bach et al., 1998). L507 is intertwined between residues T504 and R509 that are involved in glutamate binding. Crystallographic studies have shown that the leucine residue forms a hydrophobic cluster with the conserved residues Leu-748 and Leu-751 between adjacent receptor subunits (Armstrong and Gouaux, 2000). This point mutation was used as a marker for desensitization (see section 3.2.1, Figure 1B). 
The other regions involved in AMPA/kainate receptor desensitization include, $\mathrm{S} 650$ at the $\mathrm{N}$ terminus of $\mathrm{S} 2$ domain in GluR1 (Mano et al., 1996) and the corresponding site A689 of GluR6 (Swanson et al., 1997a) which when mutated display altered kinetics of desensitization.

\subsection{The role of post transcriptional modifications of AMPA and kainate receptors in gating}

\subsubsection{RNA editing}

Glutamate receptors of AMPA/Kainate subtype undergo post-transcriptional modification under various developmental and physiological conditions. Nuclear RNA editing is a physiological process, which occurs in nucleus prior to the removal of introns. It is carried out by site selective (Seeburg, 1996) deamination of adenosine to inosine (Bass et al., 1997) that is mediated by a candidate mammalian editing enzyme ADAR2 (adenosine deaminase) (Bass et al., 1997; Melcher et al., 1996). The genomic DNA sequence encoding the critical position of channel pore (M2 loop) of AMPA and kainate receptor subunits harbors either a glutamine codon (CAG) or an arginine codon $(\mathrm{CGG})(\mathrm{Q} / \mathrm{R}$ site), which represents an ion channel determinant. Among the AMPA receptor subunits, only GluR2 receptors are edited efficiently $(\sim 100 \%)$ from a glutamine residue to an arginine throughout postnatal development at position 607 (Q/R site) in the M2 reentrant loop after E14 in rats (Seeburg et al., 1998). mRNA editing has a number of dramatic physiological consequences (Sommer et al., 1991). The edited homomeric GluR2(R) receptors carrying positively charged arginine residue in the channel pore region form single channel with low conductivity (Swanson et al., 1997b). They are calcium impermeable (Burnashev et al., 1992; Hume et al., 1991) and have approximately linear current-voltage (I-V) relationship (Hume et al., 1991; Verdoorn et al., 1991; Washburn et al., 1997). However homomeric expression of the unedited version of $\operatorname{GluR2}$ or $\operatorname{GluR}(1,3$, or 4$)$ subunits carrying glutamine residue in the channel pore region yields high conducting channels, which are calcium permeable. Calcium permeability in neurons is thus controlled by regulating the expression GluR2(R) subunit at specific regions of brain for performing a specific physiological function. As for example, CA3 hippocampal neurons in CA3 region expresses high level of GluR2 subunit and possess heteromeric AMPA receptors that have low calcium permeability. Contrary to this, Bergman glial cells of the cerebellum, which bear undetectable GluR2 expression levels, exhibit 
highest calcium permeability (Geiger et al., 1995). The reduction of editing at $Q / R$ site results in epilepsy and early death (Brusa et al., 1995).

RNA editing is not only confined to $\mathrm{Q} / \mathrm{R}$ site within the channel pore region but is also observed in a second site within the extracellular loop linking TM3 and TM4 (Lomeli et al., 1994). In exception to GluR1 subunit, the others (GluR2, 3,4) have an arginine to glycine editing $(\mathrm{R} / \mathrm{G})$ site mediated by the enzymatic activity of ADAR1 or ADAR2 enzymes. The extent of editing shows a decrement for GluR4 subunit $(\sim 50 \%)$ as compared to GluR2 and GluR3 mRNA, which is edited to nearly $80-90 \%$. Physiological importance of this editing site lie in the faster recovery of the receptors from desensitized state, which is nearly two times as compared to unedited subunits. This alteration within the recovery time interval between unedited and edited subunits makes them to discriminate the current amplitudes of first response as compared to the second one in a train of presynaptic action potentials. This is because only edited subunits will recover faster than unedited subunits and hence are prone to get activated earlier (Lomeli et al., 1994).

Kainate receptors of only GluR5 and GluR6 subtypes, similar to GluR2 subunit of AMPA receptors undergo posttranscriptional editing from neutral glutamine residue to positively charged arginine at $(\mathrm{Q} / \mathrm{R})$ site in channel pore region of TM2. The extent of editing is developmentally regulated in the rat brain. Unlike GluR2 subunit, significant proportions of kainate receptors remain unedited both at embryonic level and adult stages of CNS (Dingledine et al., 1999).

The editing in kainate receptor subunits GluR5 and GluR6 is not only pertained to channel pore region but also occurs in the hydrophobic transmembrane region $\mathrm{M} 1$ at the $\mathrm{I} / \mathrm{V}$ and $\mathrm{Y} / \mathrm{C}$ sites where isoleucine is changed to valine and tyrosine residue is changed to cysteine (Kohler et al., 1993). Although eight different edited variants of GluR6 subunits are found to be present to varying extents in the CNS, the fully edited GluR6 (R/V/C) is the most abundantly expressed in the adult CNS (Kohler et al., 1993). Editing at the Q/R site in GluR6 (Burnashev, 1996) controls anion permeability (Burnashev, 1996).

\subsubsection{Alternative splicing}

The diversity of non-NMDA receptors is further enhanced by alternative splicing. All four AMPA receptor subunits occur in two alternatively spliced versions, flip and flop, that are encoded by exons 14 and 15 positioned just before the 
transmembrane M4 domain (Monyer et al., 1991; Sommer et al., 1990). A cassette of 34 amino acids located extracellularly constitute the flip/flop module. The flip variants predominate before birth and continue to be expressed in adult rats through out the life, whereas flop variants show low abundance before the eighth postnatal day. In adult animals, both isoforms are regulated to the same level. Hence the molecular basis of alternative splicing appears to play an important role in discriminating the developing brain from the developed ones. The flip/flop module affects the rate of receptor desensitization (Mosbacher et al., 1994). Both subunit type and flip/flop region are some of the controlling factors of rate of desensitization. The variation in alternative splicing affects the biophysical properties of the receptors and furthermore the effect of modulators on the kinetics of desensitization in these receptors varies dramatically. For example, cyclothiazide blocks the desensitization of flip isoforms effectively, while PEPA (4-[2(phenylsulfonylamino)ethylthio]2,6difluoro-phenoxyacetamide) preferentially reduces the desensitization of the flop forms.

During development, the faster desensitizing flop versions is offset by the increased occurence of less desensitizing R/G edited receptor variants (Lomeli et al., 1994). Heteromeric receptors lacking GluR2 subunit, yet dominated by GluR4 flop version have extremely rapid channel kinetics (Geiger et al., 1995; Mosbacher et al., 1994).

Kainate receptors of GluR5 subtype display four different C-tails and, additionally, an exon encoding 15 amino acids in the $\mathrm{N}$ terminus occurs in some transcripts (Gregor et al., 1993; Sommer and Seeburg, 1992). Alternative splicing of GluR5 subunit results in two splice variants GluR5-1 and GluR5-2 which differs either in the presence or absence of additional 15 amino acids cassette in the extracellular N-terminal region (Bettler et al., 1990) before the S1-domain. GluR5-1 with additional 15 amino acid cassette alternatively splices into two variants GluR5A and GluR5B that differs in the length of C-terminus tail. GluR5-2 undergo further splicing into three alternative varying $\mathrm{C}$-terminal sequences. Additional exons located in the C-terminal domain give rise to GluR5-2a and GluR5-2c. GluR5-2a is 49 amino acids shorter than the original GluR5-2 (GluR5-2b) because of the introduction of a premature stop codon, whereas GluR5-2c contains an extra in-frame exon that makes it 29 amino acids longer than the GluR5-2b isoform (Sommer et al., 1992).

Functional characterization among these different splice variants have not yet 
been reported. The differences in $\mathrm{C}$ termini may result in differential binding to various intracellular proteins and thus may influence receptor targeting. In this study, we tried to characterize the biophysical properties of different isoforms of GluR5 and GluR6 subunit with a myc-epitope tag at the extracellular N-terminus region. We show that the introduction of an epitope into the receptor does not alter the biophysical properties significantly.

\subsection{Implication of receptor desensitization and deactivation in synaptic transmission}

Receptor desensitization is a striking and functionally relevent phenomenon that occur in molecules as diverge as ion channels and participate in determination of amplitude, duration, and the frequency of postsynaptic signalling within and between cells at fast chemical synapses. Katz and theseleff in 1950 reported the phenomenon of desensitization at neuromusucular junction in 1950s. Both invertebrate and vertebrate glutamate receptors undergo rapid desensitization (Dudel et al., 1990) with sudden exposure to glutamate. An increase in the concentration of the agonist determines the population of the receptors to be present in the activated or desensitized state. This is because with a slow rise in the concentration of glutamate, the channel population is desynchronized by redistribution of the receptors between bound, open and desensitized state (as shown in the gating mechanism under section 1.5.2, figure 3).

Receptor deactivation and desensitization shape the decay phase of rapid postsynaptic current (Colquhoun et al., 1992) although the relative contribution may be attributed to various physiological stimulus paradigms. At central synapses, receptor desensitization is effectively promoted by glutamate concentrations much lower than which is required for the receptor activation. A brief (1ms) pulse of saturating concentration of glutamate can mimic the shape of mEPSCs. Desensitization in AMPA receptors is nearly five fold slower than mEPSC [Trussell, 1989 \#104; Colquhoun, 1992 \#184; Raman, 1994 \#181; Hestrin, 1992 \#309; (Hestrin, 1993). Receptor desensitization at synapse insures a brief mEPSC even if the time course of glutamate in the synaptic cleft is prolonged by number of physiological factors like restricted diffusion of glutamate, poor uptake, multivesicular release or intra synaptic crosstalk (Tong et al., 1995; Trussell et al., 1993). 
Desensitization can be assayed by several methods like determining the glutamate sensitivity immediately after an evoked postsynaptic current (EPSC), uncaging of caged form of glutamate etc. At large end-bulb synapses, single EPSCs induces $35-40 \%$ reduction in the sensitivity of AMPA receptors (Otis et al., 1996). Recovery from desensitization can be studied by utilizing paired pulse experiments. In paired pulse experiments, a conditioned short pulse of agonist is followed by the test pulses at successive time intervals. The amplitude of the second response is compared to the first after normalization.

Defining in kinetic terms, the slow recovery from desensitization of the receptor is the rate limiting step during transition of the receptors to the unbound state. Any modulator or drug that interact with the process of desensitization also potentiates the EPSCs and in overall influence the local neuronal circuit and synaptic plasticity (Jones and Westbrook, 1996; Nagarajan et al., 2001).

Receptor desensitization may have direct or indirect correlation in regulating the size and shape of the synaptic current (Trussell and Fischbach, 1989; Trussell et al., 1988) and is dynamically regulated (Valenta et al., 1993). The size and shape of the decay phase in an EPSC is also sensitive to a number of factors such as the clearance of glutamate (Otis et al., 1996), glutamate uptake by various transporters (Barbour et al., 1994; Mennerick and Zorumski, 1994; Otis et al., 1996), time course of glutamate release (Higgs and Lukasiewicz, 1999), spatiotemporal glutamate profile in the synaptic cleft (Barbour et al., 1994; Neher and Sakaba, 2001), transmitter diffusion rates and dendritic filtering (Hausser, 2001).

Receptor desensitization limits the frequency at which a full amplitude of the response can be obtained from glutamate-bound state of the receptor. This is accounted by double pulse of glutamate where the amplitude of the second response becomes smaller than the first and requires a time span of tens of milliseconds to recover to the activatable (unbound) state of the receptor. Both positive and negative implications are expected by receptor desensitization based on various physiological conditions. As for example, the intensification of synaptic depression during high frequency train of action potentials at least at the calyx of held synapse (Neher and Sakaba, 2001; Otis et al., 1996; Trussell et al., 1993) is due to AMPA receptor desensitization. Thinking in positive terms, receptor desensitization may act as a negative feed back mechanism to prevent undesirable effects caused by excess activation of the receptor during high frequency stimulus under abnormal 
physiological conditions especially during excitotoxicity. Desensitization thus act as an important inbuilt mechanistic switch in the receptor that integrates the collective information from single site by the neuronal input (excitatory or inhibitory) and controls the output frequency of the transmission through postsynaptic receptors. Hence firing pattern of the neuron can be switched on or off based on the input-output frequency which is dependent both on concentration of glutamate released and the population of the receptors that undergo desensitization.

Apart from the dependency of concentration and structural variations of the agonist for the receptor, several other endogenous mechanisms within the channel itself might also alter the properties pertaining to desensitization. As for example, receptor phosphorylation by protein kinases may alter the gating kinetics. These mechanisms are best understood in phototransduction molecular systems (Knox et al., 1993; Thurmond et al., 1997) or G-protein coupled receptor where phosphorylation is a primary event in the initiation of desensitization either in homologous or heterologous fashion (Hosey, 1999).

\section{Material and methods}

\subsection{Chemicals and solutions employed}

\subsubsection{Electrophysiological studies}

If not otherwise stated chemicals and drugs were purchased from Sigma, Germany. The following chemicals were employed for electrophysiological experiments.

\section{(A) Standard external solution (in mM)}

$\begin{array}{lrl}\mathrm{NaCl} & 170 & \\ \mathrm{CaCl}_{2} & 4 & \text { (BDH Laboratory Supplies, England) } \\ \mathrm{MgCl}_{2} & 4 & \text { (Merck, Germany) } \\ \mathrm{KCl} & 2.4 & \\ \mathrm{HEPES} & 10 & \\ \text { Glucose } & 10 & \\ \text { (pH 7.25, Osmolarity; } 355 \text { mOsm) }\end{array}$


(B) Standard internal solution (in mM)

$\begin{array}{ll}\mathrm{NaCl} & 10 \\ \mathrm{CsF} & 150 \\ \mathrm{HEPES} & 20 \\ \text { EGTA } & 10 \\ \mathrm{~K}-\mathrm{Gluconate} & 125 \\ \mathrm{MgCl}_{2}, & 4.5 \\ \text { ATP-Na2, } & 4 \\ \text { Creatine phosphate } & 15 \\ \text { Phosphocreatine kinase } & 20 \mathrm{U} \mathrm{ml}^{-1} \\ \text { (pH 7.25, Osmolarity; } 330 \mathrm{mOsm}^{2}\end{array}$

(C) Drugs added in the extracellular solution

Drugs were added to the standard extracellular solution to obtain following final concentrations:

$\begin{array}{lrl}\text { TTX } & 0.2 \mu \mathrm{M} & \\ \text { Glutamate } & 10 \mathrm{mM} & \\ \text { D-AP5 } & 50 \mu \mathrm{M} & \\ \text { CTZ } & 100 \mu \mathrm{M} & \text { (Tocris, USA) } \\ \text { Quisqualate } & 100 \mu \mathrm{M} & \text { (Tocris, USA) } \\ \text { IDRA 21 } & 300 \mu \mathrm{M} & \text { (Tocris, USA) } \\ \text { CX516 } & 300 \mu \mathrm{M} & \text { (Tocris, USA) } \\ \text { CX546 } & 300 \mu \mathrm{M} & \text { (Organon, UK) }\end{array}$

(D) Drugs added to the intracellular solution

Drugs were added to the standard intracellular solution to get the final concentrations:

Spermine $\quad 50 \mu \mathrm{M} \quad$ (Tocris, USA)

(E) Solvents employed for the experiments

DMSO was employed for dissolving Cyclothiazide, CX546, CX516 and IDRA 21. Otherwise water was used. 


\subsubsection{Cell culture}

\section{Cell coating}

Poly-D-Lysine

Collagen (Becton Dickinson Biosciences, Germany)

Acetic acid (Glacial)

\section{Cell culture}

RPMI 1640

(GIBCO BRL, UK)

Kynurenic acid

Pencillin-Streptomycin (Roche Diagnostics GmbH, USA)

\section{Transfection chemicals}

BES (N,N-bis[2-Hydroxyethyl]-2-aminoethanesulphonic acid)

$\mathrm{Na}_{2} \mathrm{HPO}_{4} \cdot 12 \mathrm{H} 2 \mathrm{O}$ (Merck, Germany)

\subsection{Preparation of solutions and procedures employed in cell culture and coating}

\section{Procedures for coating}

Glass coverslips (plates) are cleaned before use. The coverslips are gently swirled in $1 \mathrm{~N} \mathrm{HCl}$ solution overnight on a lab shaker. The coverslips are rinsed with double distilled water several times till they are acid free. The coverslips are rinsed once or twice in $70 \% \mathrm{EtOH}$, and then store them in $95 \%$ EtOH. Perhaps the most tedious part of the whole process is placing the plates into the culture dishes. Each coverslip is grasped with forceps, flame dried, and placed into its own well. The flame drying is quicker and supposed to have some beneficial effect caused by the fine residue of carbon left behind as the alcohol burns. Once the plates are dry and in culture wells they are ready to be coated.

A mixture of glacial acetic acid $(17 \mathrm{mM})$, poly-D-lysine $(0.5 \mathrm{mg} / \mathrm{ml})$, collagen $(3.11 \mathrm{mg} / \mathrm{ml})$ are mixed at ratio 3:1:1 and used for coating on the glass cover slips. 
Irrespective of the number of wells in the culture plate (6 or 12 wells plate), the procedure for coating was similar. The culture plate with coverslips are now sterilized in the UV chamber for 30 minutes.

\section{Procedures for cell culture}

Human embryonic kidney cell line HEK293 (ATCC, USA) were cultured in RPMI 1640 (Gibco BRL) supplemented with 10\% fetal calf serum and $400 \mu \mathrm{l}$ of Penicillin/ Streptomycin (Penicillin, 50,000U/ml and Streptomycin 50ng/ml). The medium was additionally containing kynurenic acid. The cells were passaged up to 15 times before starting a new passage. Transfections were carried out 24 hours after the harvestment. We exchanged the cell culture medium to fresh medium before starting the transfection.

\section{Procedures for transfection}

We used calcium phosphate based transfections in all the experiments as desribed previously by Chen et al (Chen and Okayama, 1988). Cells were plated at a density of $2 \times 10^{5}$ per $35 \mathrm{~mm}$ dish using a total concentration of $3 \mu \mathrm{g}$ cDNA encoding the wild and mutant AMPA receptors. Transfected cells were detected by cotransfecting with $0.1 \mu \mathrm{g} / \mu \mathrm{l}$ of the plasmid encoding cDNA for green fluorescent protein. Cells were washed with RPMI 1640 medium 6-8 hr after transfection, and were used for electrophysiological recordings after $24 \mathrm{hr}$. Neurons were cultured as described in (Bekkers and Stevens, 1991) and were used for recordings 1-2 weeks after plating.

\subsection{Receptor used in the experiments}

Flip and flop versions of GluR1 and GluR2 subunits were used for the experiments concerning AMPA receptors. Both edited and unedited subunits $(\mathrm{Q} / \mathrm{R})$ of AMPA subunits have been used in this work. These receptors were kindly granted by Dr. Peter Seeburg, Heidelberg Germany. We have further employed the extracellular myc epitope tagged alternatively spliced variants of GluR5 and GluR6 subunits that 
were kindly provided by Dr. Christoph Mulle, Université Bordeaux2, Bordeaux, France.

\subsection{Mutagenesis methodology}

cDNAs encoded for the alternatively spliced flip version of wild type GluR1(Q) and GluR2 (Q/R) subunits (Sommer et al., 1990) were employed for all the experiments. While point mutations were introduced using the PCR-based method described by the 'QuickChange' mutagenesis manual (Stratagene). The L-Y point mutation was introduced at position L497 in the GluR1 subunit, and at the corresponding position in the GluR2 subunit (L504). Similar point mutations were made at the Q/R site in GluR2 (Q607) and corresponding site on GluR1 subunit subunit to introduce arginine residue in the channel pore region in the position of glutamine.

The kainate receptors of GluR5 and GluR6 subtype carrying either glutamine or point-mutated arginine were employed in this study. The deletion mutants of GluR6 subunit lacking 4, 14 and 54 (R6-4, R6-14, R6-54) amino acids were employed in our experiments for characterizing the biophysical properties. Furthermore, we also characterized the gating properties of various isoforms of GluR5 subunit (GluR5A and GluR5B) and determined the effect of removing 15 amino acids in N-terminus region before the S1-domain.

Mutations were verified by double-strand DNA sequencing. All receptors were subcloned into pRK5 vectors (InVitrogen). Amino acid numbering starts from the first methionine of the open reading frame. Mutagenesis was performed in collaboration with Dr. Yael Stern-Bach, Jeruselem, Israel, Dr. Ralph Nehring in the department of Membrane Biophysics, Goettingen, Germany, Franquoise Coussen and Christoph Mulle from Université Bordeaux2, Bordeaux, France.

\subsection{Biochemical analysis}

For quantitative western blot analysis of glutamate receptor expression, HEK293 cells were transiently transfected with plasmids coding for GluR1 (Q) and GluR2 (R) either alone or at the indicated ratios by Lipofectamine transfections (Life Technologies, Gaithersburg, MD). After 60 hr, cells were washed briefly with PBS, harvested, and homogenized in ice-cold lysis buffer $(50 \mathrm{mM}$ Tris/ $\mathrm{HCl}, \mathrm{pH}$ 7.4, 150 $\mathrm{mM} \mathrm{NaCl}, 1 \%$ NP-40, 0.5\% Deoxycholate, protease inhibitors $[1 \mu \mathrm{g} / \mathrm{ml}$ leupeptin, 
$1 \mu \mathrm{g} / \mathrm{ml}$ pepstatin A, $2 \mu \mathrm{g} / \mathrm{ml}$ aprotenin, and $1 \mathrm{mM}$ PMSF]). Nonsoluble components were removed by centrifugation at $4^{\circ} \mathrm{C}$ and protein concentration was determined. Equal amounts of proteins were used for SDS-PAGE and blotted onto nitrocellulose membrane. The recombinantly expressed glutamate receptors were probed with polyclonal rabbit Anti-Glutamate Receptor 1 (1:5000, Chemicon, Temecula, CA) and polyclonal rabbit Anti-Glutamate Receptor 2/3 (1:3000, Calbiochem, Cambridge, MA). Blots were developed using horseradish peroxidase-conjugated affinipure goat Anti-Rabbit IgG (1:10000, Jackson ImmunoResearch Laboratories, West Grove, PA) together with the $\mathrm{ECL}^{\mathrm{TM}}$ western blotting detection reagent (Amersham Pharmacia Biotech, Piscataway, NJ), and chemoluminescence was documented on Hyperfilm ${ }^{\mathrm{TM}}$ $\mathrm{ECL}^{\mathrm{TM}}$. Quantification of protein expression including calibration was determined using the Densitometric Analysis of 1-D Gels macro from the image analysis program NIH Image 1.62. Calibration was performed by loading relative amounts $(75 \%, 50 \%$, $25 \%$ ) of homomeric expressed GluR1 and GluR2, and signals were plotted on the xaxis against the signals from the cotransfections performed in parallel $(4: 0,3: 1,2: 2$, $1: 3$, see figure 6 , section 3.6). Signals were background subtracted. The biochemical experiments described here were performed by Dr. Ralph Nehring.

\subsection{Electrophysiology}

In this part we describe the equivalent circuit for a simple cell from an electrical point of view. This is an important basic step prior to the further understanding of patch clamp technique.

\subsubsection{The equivalent circuit for a simple cell}

One can represent the properties of biological membranes and channels by a simple equivalent electrical circuit. This circuit diagram has a near equivalent electrical properties to the membrane. The phospholipid bilayer of the membrane acts as a capacitor while the channel acts as a conductor with a resistance $\mathrm{R}$ in the order of $1 \mathrm{G} \Omega$ and input capacitance (C) on the order of $\sim 10 \mathrm{pF}$. The rate of flow of ions across the plasma membrane is determined by a) the concentration gradient ie., the difference in the concentrations of the conducted ion on the two sides of the membrane; b) the voltage difference across the membrane; c) the permeability of the 
ion ie., the ease with which it moves through its ion channel across the membrane; $d$ ) number and open probability of a channel.

The voltage required to exactly oppose the flow of any given ion $\mathrm{X}$ is called equilibrium potential. The currents through the channel pore equates to zero at the equilibrium potential of a particular ion. The equilibrium potentials for living cell is given by Nernst equation:

$$
\mathrm{Ex}=\mathrm{RT} / \mathrm{zF} * \ln [\mathrm{X}]_{\mathrm{O}} /[\mathrm{X}]_{\mathrm{i}}=2.303(\mathrm{RT} / \mathrm{zF}) \log _{10}[\mathrm{X}]_{\mathrm{o}} /[\mathrm{X}]_{\mathrm{i}}
$$

Where $\mathrm{R}$ is the gas constant, $\mathrm{T}$ is the absolute temperature in degrees kelvin, $\mathrm{z}$ is the charge on the ion, $\mathrm{F}$ is the faraday constant (the amount of charge in coulombs carried by a mole of monovalent ions).

This equation describes the relationship between voltage across a semipermeable membrane and equilibrium ion concentration. The equilibrium potential of a particular ion varies linearly with the absolute temperature and logarithmically with the concentration of ions $[\mathrm{X}]$ at the exterior $[\mathrm{Xo}]$ and interior surface of the membrane[Xi]. The sign of the equilibrium potential depends on the charge of the ions or the direction of gradient. It equates to zero when there is no gradient. One can thus compare the gradient as a battery with electromotive force Ex in series with the resistor. As for example in a neuronal cell, the equilibrium potential of $\mathrm{Na}^{+}$ions is $+67 \mathrm{mV}$ while that of $\mathrm{K}^{+}$ions is $-98 \mathrm{mV}$.

According to ohms law, the current flow between two points depend on the voltage difference across the membrane (E) and resistance to current flow (R) between those points. This is given by the equation $\mathrm{E}=\mathrm{IR}$. For the flow of an ion $\mathrm{X}^{+}$ across the membrane, the relative voltage difference will be (Vm-Ex). This represents the net driving force for a particular ion through the channel pore. Thus ohms law get deduced to $\mathrm{I}=\mathrm{g}_{\mathrm{x}}(\mathrm{Vm}-\mathrm{Ex})$ where Ex is the electromotive force in the pore, $\mathrm{Vm}$ is the voltage difference across the membrane and $\mathrm{g}_{\mathrm{x}}$ is the electrical conductance of a particular ion (inverse of electrical resistance). 


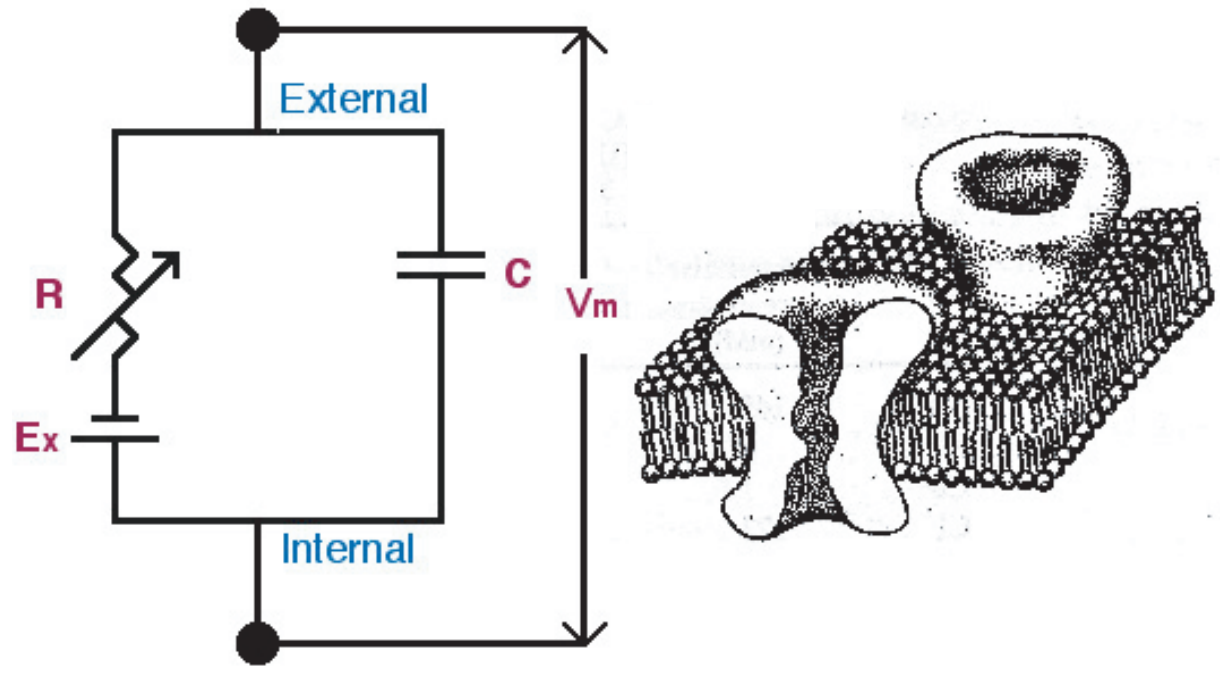

Modified from Ion channels of excitable membrane, B. Hille 3rd edition.

\section{Figure 4. Electrical equivalent of cellular membrane}

The left panel represents theelectrical circuit which is compared to the cellular membrane(right panel). The membrane acts like an equivalent circuit with a conductive and capacitive branch connected in parallel to each other. A resistor (R) with an electromotive force of Ex (battery) are in series with each other. The capacitive branch is compared to the lipid bilayer of cellular membrane. Vm represents the voltage difference between external and internal part of the membrane.

\subsubsection{An overview of Patch Clamp technique}

Patch clamp technique is an important method for studying electrophysiological properties of biological membranes which was developed by Erwin Neher and Bert Sakmann. It allows the recording of macroscopic whole-cell and microscopic single channel currents flowing across biomembranes through ion channels. With an unparalleld resolution, one can study membrane-contained ion channels, receptor activated channels (receptors activated by hormones, neurotransmitters, mechanical stress, exogenous molecules) and second-messenger activated channels (channels activated by intracellular calcium, cAMP, cGMP, $\mathrm{IP}_{3}, \mathrm{G}-$ proteins).

Using this technique, one can experimentally control and manipulate the voltage of whole cell or membrane patches. The technique has unique features to 
study the voltage dependence of ion channels (voltage-clamping) or to montior the changes in membrane potential in response to currents flowing across ion channels (current-clamp). Furthermore, the technique is suitable to determine the electrical parameters such as cell membrane capacitance that represents the surface area of the plasma membrane. The time-resolved monitoring of cell capacitance can be used to study the exo and endocytotic activity of single secretory cells (Lindau and Neher, 1988).

The electrical circuit used in the patch clamp technique is shown in the following figure.

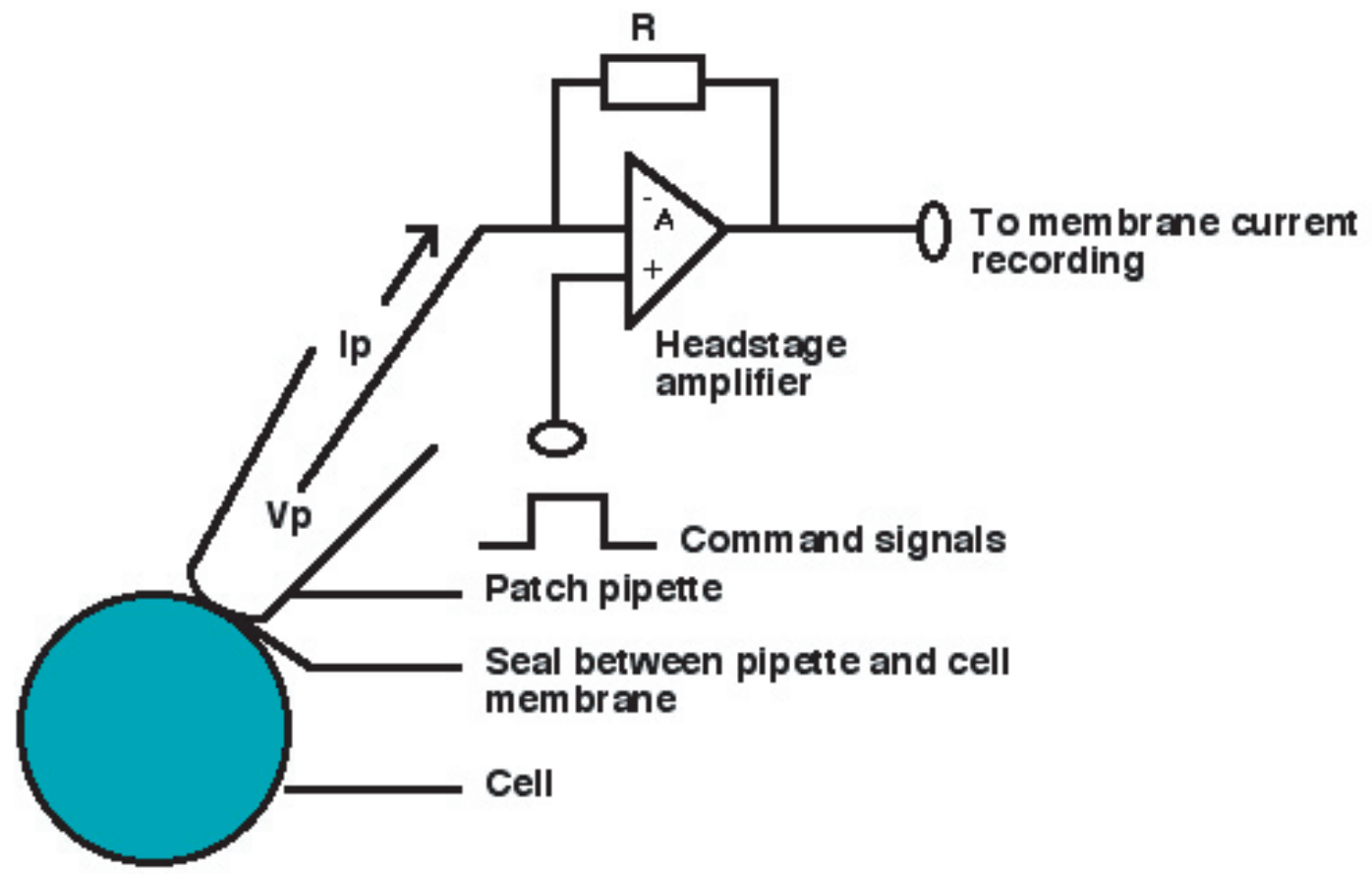

Figure 5. Schematic representation of patch clamp circuit

The diagram illustrates the circuits in the patch clamp technique. The patch pipette is in direct contact with the cellular membrane to form a seal. The current flowing through the ion channels on the membrane enters the patch pipette through an electrode. With pipette potential of $\mathrm{Vp}$, the current through it (Ip) is then fed to an current-voltage converter (head stage amplifier) which has a feedback resistor $(\mathrm{R})$. The amplified current can be monitored by a oscilloscope

To perform patch clamp recording, a glass pipette, with an internal diameter of the order of a micron or so at the tip is placed against the cellular membrane. The patch pipette is partially filled with the filtered internal solution and then inserted into the pipette holder. Pipette holders are made up of teflon or polycarbonate having low 
dielectric loss. The pipette electrode is a thin silver wire that has chloride coating. The bath electrode is a bare chlorided silver wire.

The application of suction to the inside of the pipette lead to an electrical seal between the glass and the membrane. The process of the seal formation can be monitored by observing the change in the pipette currents on an oscilloscope while applying voltage pulses to the pipette. This results in the establishment of giga seal $\left(10^{9} \Omega\right)$ at the contact area. The formation of giga seal will cause an increase in resistance with the reduction in currents. This high resistance seal becomes so tight that ions are effectively prevented from leaking out through it. The resistance between membrane and patch pipette is crucial for determining the electrical background noise from which the channel currents are separated. Based on the size of the membrane patch and density of ion channels in the membrane under the pipette, a single or multi channel proteins can be measured under the pipette. Current that is carried by influx or efflux of ions through the channel can be detected by a sensitive current-voltage converter (patch clamp amplifier) and is connected to the inner part of the electrode. The amplifier converts small patch pipette currents (pico to nanoamperes) into voltage signals that can be observed by oscilloscope or sampled by a computer. It has a high gain, owing to the large feedback resistor R. The potential inside the pipette, Vref (command signals) may be held at a steady level or changed in a step-wise fashion by pulses from an external source (Vcommand). The feed-back resistor determines the sensitivity and range of current measurement and sets the gain (Vout/ $i_{p}, \mathrm{mV} / \mathrm{pA}$ ). If $\mathrm{i}_{\mathrm{p}}$ represents the patch current of the membrane then, $-\mathrm{i}_{\mathrm{p}} \mathrm{R}=$ Vout-Vref, where Vout represents the output potential and Vref is the pipette potential. Patch clamp amplifiers allows the holding potential of the patch membrane to be altered (Vhold).

\subsubsection{Configurations of patch clamp technique}

The different configurations of patch clamp recordings allow one to study different properties of channel proteins. Based on purpose of the experiment they are classified into four categories. 


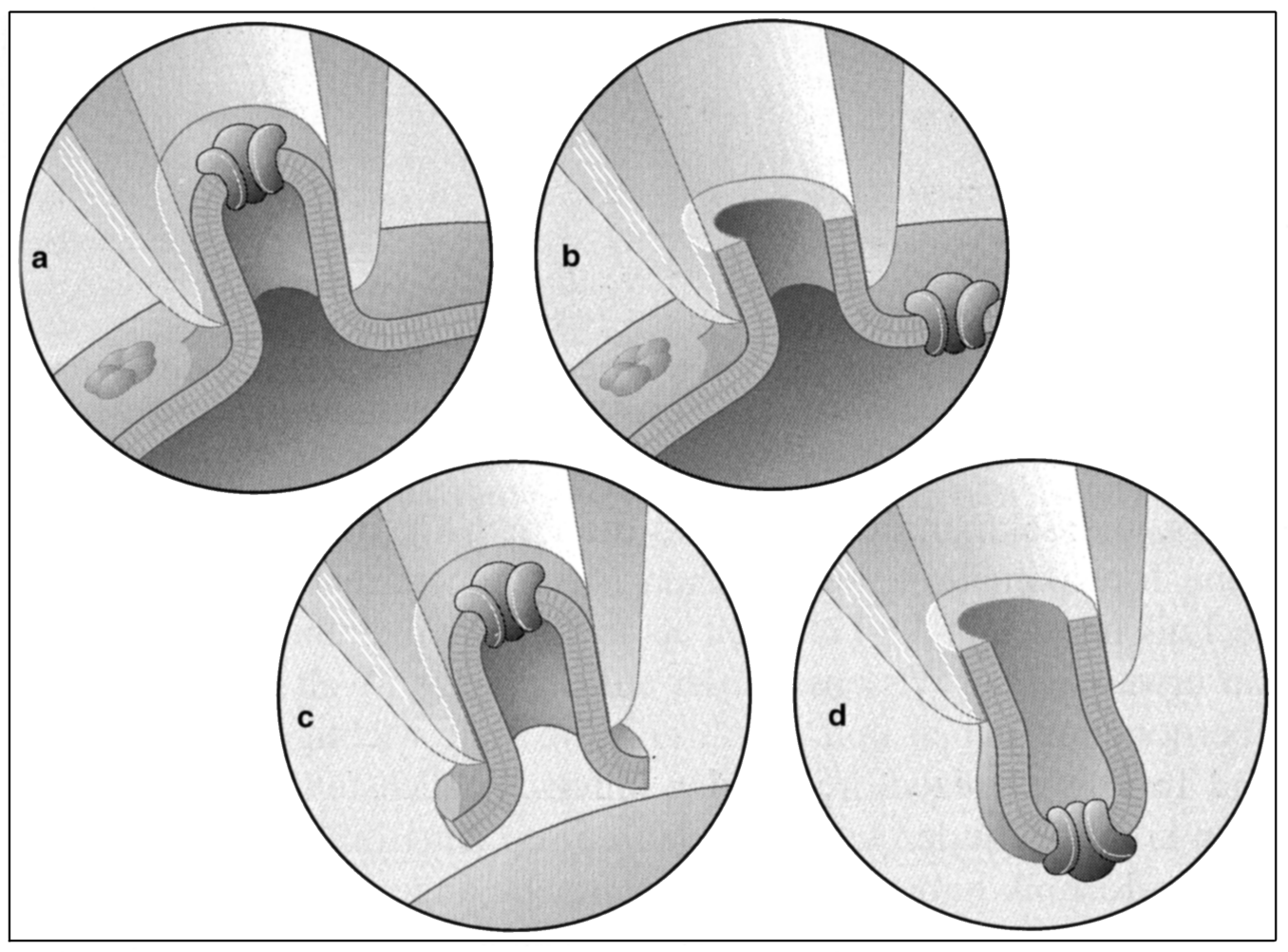

Courtesy from E. Neher

Figure 6. Schematic representation of patch clamp configurations for electrophysiological recordings

The four recording configurations used in patch clamp technique are "cell attached", "whole cell recording", "outside-out patch" and "inside out patch". The frame (a) represents the configuration of a pipette with the membrane after a slight suction is applied to the cellular membrane. The seal between membrane and pipette increases in resistance by more than two orders of magnitude leading to cell-attached patch. The membrane can be ruptured by further suction to obtain whole cell recording mode (b). Frame (c) represents the inside-out patch configuration while frame (d) corresponds to outside-out patch configuration.

\section{Cell attached mode}

This configuration mode is used when the type of the channel requires unknown cytosolic factors that would have lost during patch excision. This allows one to know whether particular kind of an ion channel is gated by diffusible second messenger. However, this method does not allow one to know the resting membrane potential of the cell that adds to the applied pipette potential. Secondly, this method is incapable of controlling the ionic composition of solutions at the inner and outer surface of the membrane patch. The seal between membrane and pipette increases in 
resistance by more than two orders of magnitude leading to cell-attached patch (as shown in the frame (a) of schematic representation).

\section{Whole cell mode}

From cell-attached mode, the membrane can be ruptured by further suction to obtain the whole-cell recording mode. This results in direct contact of intracellular part of the membrane with the pipette solution. The configuration is employed when the average current from all ion channels in the entire cell is to be recorded. It is also used to study exocytotic activity of secretory cells by determining the cell membrane capacitance. The disadvantage of this method is the possible loss of cytosolic factors (as shown in the frame (b) of figure 6).

\section{Inside out patch configuration}

From cell attached configuration mode, manipulations can be made to align the internal surface of the membrane towards the external medium. This configuration enables one to study the gating of second-messenger-activated channels. The effect of number of cytosolic signaling molecules in modulating the channel properties can thus be studied (as shown in the frame (c) of figure 6).

\section{Outside-out patch configuration}

From whole cell mode, one can excise and isolate a small portion of the membrane to obtain outside-out patch configuration. This configuration results in alignment of extracellular surface of the membrane facing towards the external medium for exchange of different ligands. Hence one can study ligand-gated ion channels in this configuration (as shown in the frame (d) of figure 6).

\subsubsection{Experimental procedures}

Given the complexity and multiplicity of receptor subtypes and their interactions, there are several advantages for using a heterologous system to examine the electrophysiological properties of receptors. Often receptors exist in alternative forms, subtypes or isoforms. Mammalian tissue culture cells such as HEK-293 are frequently employed for studying recombinant expression of individual receptors. They have the advantage of providing an environment that is similar to the milieu of the natural human cells. To a great degree, channel permeability, post-translational processing, signaling and coupling to other cellular factors in these cells are similar to the processes in most mammalian cells. Post-translational processing like protein glycosylation varies in other expression systems like Xenopus oocytes (lower 
vertebrates). This could probably interfere with the proper assembly of subunits. The other advantages include their capacity in rapidly expressing receptor proteins under test (within 24-48 hrs) and their small size that allows them to use in electrophysiological experiments.

Outside-out membrane patches were excised from HEK293 cell expressing wild-type and mutant receptors, and from primary cultured mouse hippocampal neurons. After excision of a patch, it was positioned in front of a fast perfusion system consisting of a theta tube flow pipe mounted on a piezoelectric translator (Clements and Westbrook, 1991; Colquhoun et al., 1992). The fast perfusion system is described in figure 7.

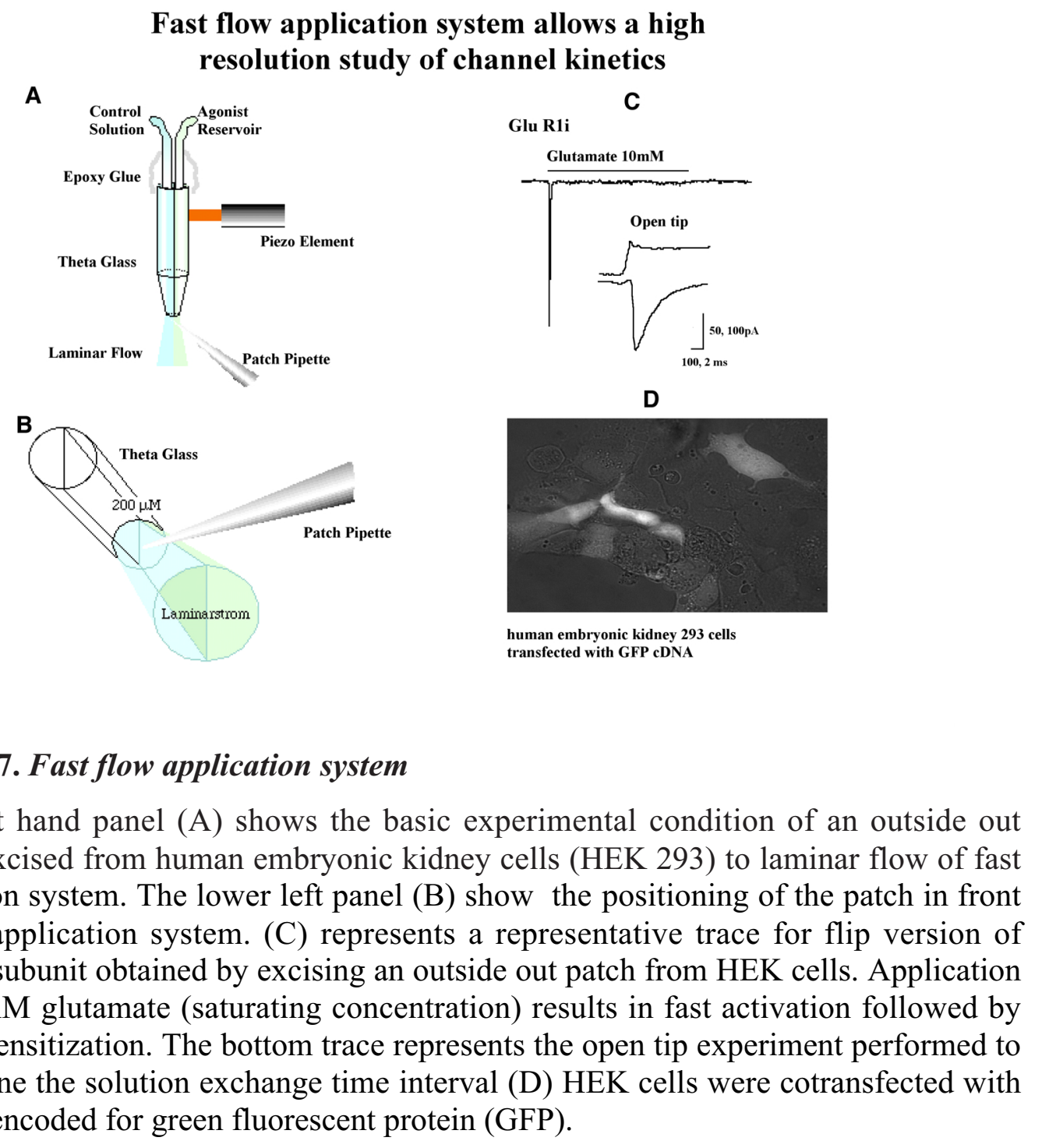

At the completion of a recording, solution exchange time (20-80\% to peak) was estimated from open tip control, which ranged from 0.3-0.6 ms (as shown in inset 
of ' $\mathrm{C}$ '). Experiments were performed at room temperature. Agonist application was repeated at 0.2-0.02 Hz. Patch pipettes had a resistance of 2-4 M $\Omega$, and were filled with the internal solution containing $150 \mathrm{mM} \mathrm{CsF}, 20 \mathrm{mM}$ HEPES, $10 \mathrm{mM} \mathrm{NaCl}, 10$ mM EGTA, adjusted to $355 \mathrm{mOsm}, \mathrm{pH} 7.3$ to which $50 \mu \mathrm{M}$ spermine was added. The extracellular medium contained $170 \mathrm{mM} \mathrm{NaCl}, 10 \mathrm{mM}$ HEPES, $10 \mathrm{mM}$ glucose, 4 $\mathrm{mM} \mathrm{CaCl}_{2}, 4 \mathrm{mM} \mathrm{MgCl}$, adjusted to $355 \mathrm{mOsm}$, $\mathrm{pH} 7.25$.

For experiments on neurons, the internal solution had the composition 125 mM K-gluconate, $10 \mathrm{mM} \mathrm{NaCl}, 4.6 \mathrm{MgCl}_{2}, 4 \mathrm{mM}$ ATP-Na2, $15 \mathrm{mM}$ creatine phosphate, phosphocreatine kinase $\left(20 \mathrm{U} \mathrm{ml}^{-1}\right), 1$ mM EGTA; buffered $\mathrm{pH} 7.3 ; 300$ mOsm. 50 $\mathrm{M}$ D-AP5 was included in external solution to block glutamate mediated NMDA. TTX $(0.2 \mu \mathrm{M})$ concentration was added to the internal solution to block the sodium currents.

Agonist solutions (glutamate and AMPA) were made by mixing external medium with isotonic agonist stock solutions (355 mOsm, $\mathrm{pH} 7.25$ ). Cyclothiazide was dissolved in DMSO before dilution with extracellular solution. Holding potential was usually $-60 \mathrm{mV}$. Voltage step protocols were used to record inward and outward currents between $-60 \mathrm{mV}$ and $+30 \mathrm{mV}$. Currents were amplified using an Axopatch amplifier $200 \mathrm{~B}$ (Axon Instr., USA), filtered at $2 \mathrm{kHz}$ and digitized at $20 \mathrm{kHz}$ using pClamp 8.0 (Axon Instr., USA) acquisition system.

\subsubsection{Epitope tagging strategy}

One is frequently faced with the difficult task of detecting the expressed protein in a variety of antibody-based experimental strategies, such as western blot, immunoprecipitation and immunocytochemistry using fluorescent-tagged antibodies. These problems could be circumvented by using epitope tagging strategy. In this strategy, recombinant proteins are expressed as constructs bearing a short oligopeptide epitope added to the protein. Antibodies directed against the added epitope can then be used as tool for detection of the protein in several biochemical experiments like Western blot, immunocytochemistry, fluorescence activated cell sorting (FACS) etc. The epitope tagging strategy offers a number of advantages over the use of antibodies generated directly against naturally occurring structural epitopes in the protein of interest. Since, epitope tagging makes use of antibodies with known characteristics, extensive characterization of new antibodies is unnecessary. Secondly, the epitope 
tagging approach may be particularly useful for discriminating among otherwise similar gene products that could not be distinguished with conventional antibodies.

In the current study we employ the myc-epitope tagged receptors of kainate receptor subunits to characterize their gating properties.

\subsection{Data analysis}

\subsubsection{Software used for the data analysis}

Analysis was performed using AxoGraph 4 software (Axon Instruments) and graphed using KaleidaGraph 3.5 (Synergy Software). The time course of desensitization was calculated from averaged episodes (4-5) after subtracting the current responses from $0 \mathrm{mV}$. Error bars indicate standard error. Statistical significance test was performed using students t-test (unpaired). IGOR curve fit program were used for performing exponential fits to determine the resensitization time constants.

\subsubsection{Statistical test for curve fits}

\section{Chi-square Goodness of fit test}

Chi-square test used in the analysis work is a statistical test to determine the deviation of theoretical frequencies from the expected frequencies. It is used test if a sample of data came from a population with a specific distribution. The chi-square goodness-of-fit test is applied to binned data (i.e., data put into classes).

The chi-square test is defined for the hypothesis:

$\mathrm{H}_{0}$ : The data follow a specified distribution.

Ha: The data do not follow the specified distribution.

For the chi-square goodness-of-fit computation, the data are divided into $\mathrm{k}$

bins and the test statistic is defined as

$$
\chi^{2}=\sum_{i=1}^{k}(\mathrm{Oi}-\mathrm{Ei})^{2} / \mathrm{Ei}
$$

Where Oi and Ei represents the observed and expected frequency for bin i. The expected frequency is calculated by using the equation:

$$
\mathrm{Ei}=\mathrm{N}\left(\mathrm{F}\left(\mathrm{Y}_{\mathrm{u}}\right)-\mathrm{F}\left(\mathrm{Y}_{1}\right)\right)
$$


Where $\mathrm{F}$ is the cumulative distribution function for the distribution being tested, $\mathrm{Y}_{\mathrm{u}}$ is the upper limit for class $\mathrm{i}, \mathrm{Y}_{1}$ is the lower limit of class $\mathrm{i}$ and $\mathrm{N}$ is the sample size. For the chi-square approximation to be valid, the expected frequency should be at least 5 .

\subsubsection{Noise analysis}

In whole cell or membrane patch recording, the current signal is composed of many overlapping single channel events. One can use analysis of current fluctuations to prevent the difficulties in detecting individual events.

One can determine single channel amplitude and mean open times using this methodology (Neher and Stevens, 1977). From fluctuating current signals, one can determine mean current and current variance if the channel open probability $\left(P_{0}\right)$ is small. Open probability is an emperical index that detemines the probability of a channel being in open state. In the steady state, $P o$ is simply the average fraction of time spent by the channel in open state. In case of recordings having one open level, the open probability will be average current level throughout the record divided by the open-channel current level.

The mean current $I$ can be equated to the product of single channel current (i), the number of channels (n) and the open probability. This is given by the equation:

$$
I=i n P o
$$

A binomial distribution can be applied to determine the variance since channels can be either in open of closed state. The variance is given by the equation:

$$
\sigma^{2}=i^{2} n p_{o}\left(1-p_{o}\right)
$$

Using equation (1) the equation for variance can be deduced to

$$
\sigma^{2}=i I\left(1-p_{o}\right)
$$

If the open probability of the channel is too small then $\left(1-p_{o}\right) \approx 1$

Thus the expression of single channel current gets reduced to the form

$$
\mathrm{i} \approx \sigma^{2} / I
$$

When nose analysis is applied to channels with several sub-conductance levels, or when several channel types are present, the conductance estimate represents the weighted average of various sub-conductances. The weighting factors represents the corresponding open probabilities. The conductance estimates from noise analysis in this study were obtained under conditions of low open probability (in the absence of cyclothiazide). 


\section{RESULTS}

\subsection{Overview}

The result section discusses three issues. The first part analyzes the AMPA receptor assembly and gating mechanisms. We used GluR1 and GluR2 subunits to test these properties. Comparison between homomeric and heteromeric assembly was studied by monitoring the desensitization and rectification properties. A nondesensitizing marker (L-Y) and a non-rectifying marker $(\mathrm{Q}-\mathrm{R})$ were designated on either GluR1 or GluR2 subunit (termed as R1 and R2 subunits hereafter). These independent receptor properties served to target the markers to structurally different parts of the subunit for probing the channel function.

Two independent point mutations (markers) that can alter the marker phenotype were introduced into these subunits. The subunits were designated to carry the marker for desensitization or rectification properties. These properties were monitored as a function of subunit composition and relative expression level. The introduction of functional markers helped to understand the molecular mechanisms involved in the assembly of AMPA receptors.

In the second part of the work we wanted to understand how the gating properties of AMPA receptors are modulated by different AMPA modulators.

The third part deals with the biophysical characterization of kainate receptor subunits of GluR5 and GluR6 subtype (termed as R5 and R6 subunits hereafter).

\subsection{Gating and assembly properties of AMPA receptors}

\subsubsection{Specific gating mechanisms}

A desensitizing wild-type AMPA receptor can be converted to a nondesensitizing phenotype by mutating a highly conserved leucine residue to an aromatic tyrosine residue in the extracellular ligand-binding domain (R1(L497); R2(L504); R3(L507)) (Robert et al., 2001; Stern-Bach et al., 1998; Takamori et al., 2000; Thalhammer et al., 1999). This residue is conserved in all the subunits of AMPA receptor. A typical trace of a desensitizing receptor and nondesensitizing receptor is shown in Figure 1. 


\section{TM1}

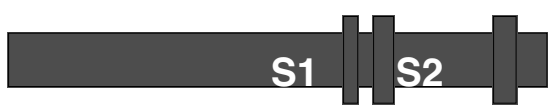

\section{Glutamate $10 \mathrm{mM}$}

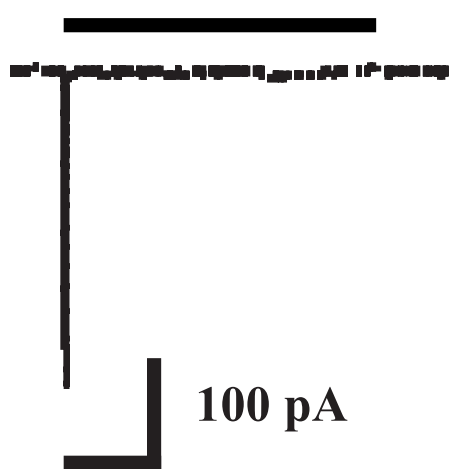

$100 \mathrm{~ms}$

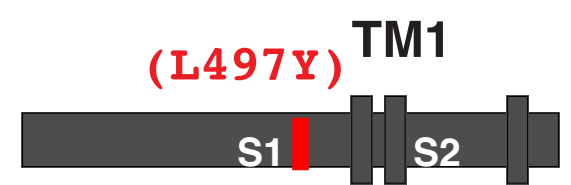

\section{Glutamate $10 \mathrm{mM}$}

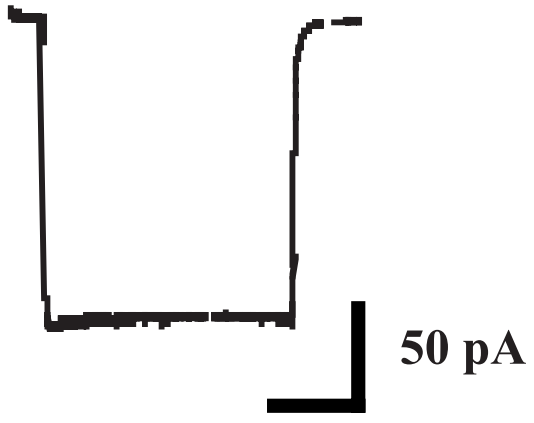

$100 \mathrm{~ms}$

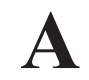

B

Figure 1. Desensitization characteristics of wild-type and mutant receptors

The left hand panel (A) is a typical raw trace of an electrophysiological response obtained from an outside-out patch excised from HEK293 cell expressing R1 subunit (AMPA receptor subtype). Application of $10 \mathrm{mM}$ glutamate to wild-type GluR1 receptors results in fast activation followed by fast desensitization. The membrane potential was voltage clamped and the holding potential was $-60 \mathrm{mV}$. On the top is a cartoon of the primary protein sequence with transmembrane regions (TM1) being represented as black vertical bars. The horizontal bar represents the time of glutamate application. S1 and S2 correspond to the domains of the ligand binding pocket. TM1 represents the transmembrane domain 1. The right hand panel (B) shows the effect of point mutation in S1 region. Mutating the highly conserved hydrophobic residue leucine to aromatic residue tyrosine (L497Y in R1 subunit) results in complete block of desensitization.

In AMPA receptors the ion permeation is controlled by the presence of an arginine/glutamine in the pore forming region of the channel. Only R2 subunit undergoes RNA editing from glutamine to arginine (Q607R) in the channel pore region. AMPA receptor lacking R2(R) subunit allow cation influx through the channel but not the efflux. This property of the channel to conduct ion permeation through the channel in one direction (influx) is called inward rectification. In neuronal cells the inward rectification is caused due to intracellular block of channel by cytosolic polyamines such as spermines at positive membrane potential. Furthermore, the 
inward rectification can be abolished by arginine carrying subunits in the assembled channel. This is mainly due to relief of the intracellular spermine block due to electrostatic repulsion (Bowie and Mayer, 1995; Burnashev et al., 1992; Donevan and Rogawski, 1995; Kamboj et al., 1995; Koh et al., 1995). Such channels conduct ions in a linear fashion. This is explained in figure 2.

\section{Editing site in the channel pore modifies rectification}
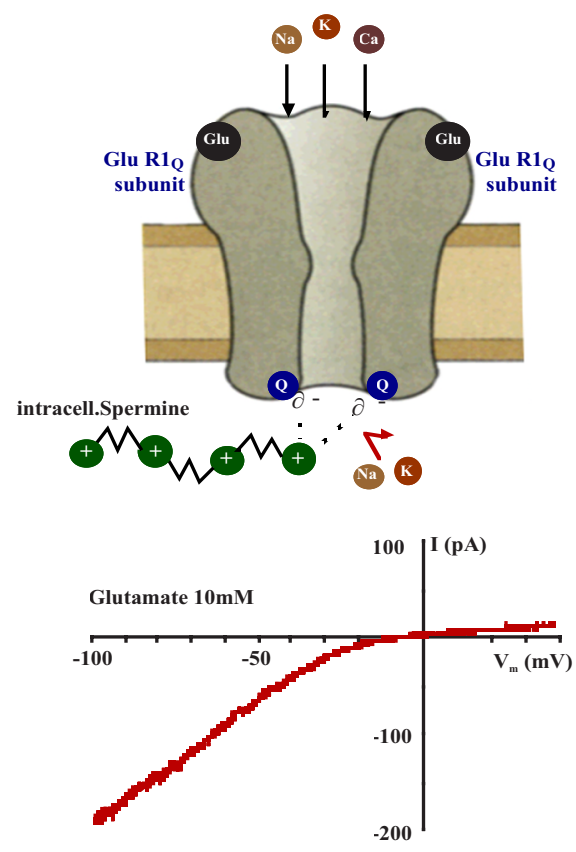
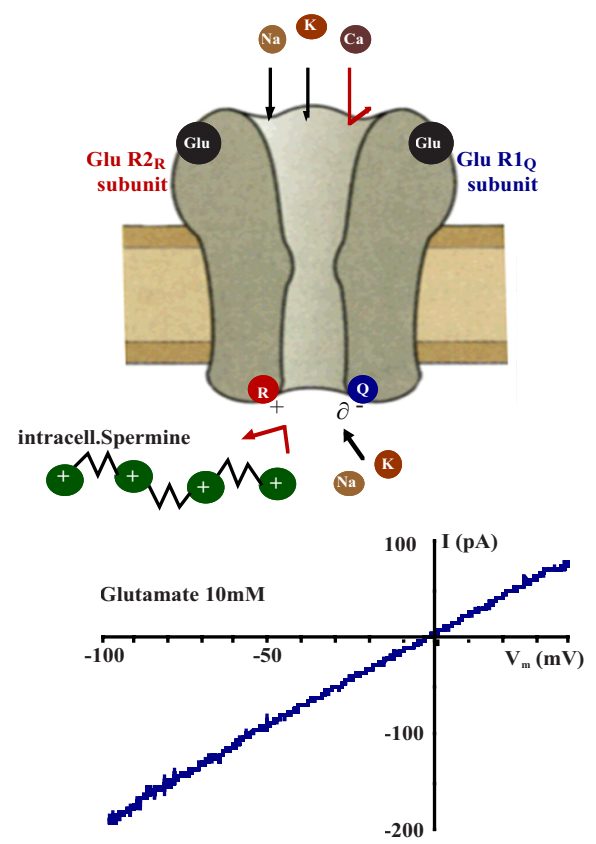

\section{Figure 2. $R N A$ editing and rectification properties}

The cartoon (left panel) describes the homomeric GluR1 subunit carrying glutamine residues in the channel pore region. The channel is in the open conformational state due to binding of the glutamate. In the open conformation the channel conduct monovalent and divalent cations (sodium, potassium and calcium). The channel pore region is blocked by intracellular spermines preventing the flow of cation in outward direction (inward rectification). The figure below describes the conducting properties of homomeric $\mathrm{R} 1(\mathrm{Q})$ receptor in terms of current-voltage relationship. In the region of negative membrane potential, the current flow is linear implying that the ion permeation through channel is not disturbed by any endogenous molecules like spermines. Towards the positive membrane potential the direction of current changes from linear to inward rectification. The inward rectification is due to the block of the channel by endogenous spermines at positive membrane potential resulting in 
preventing the cation efflux. Shown on the right panel is a heteromeric receptor carrying R1 and R2 subunits. R1 subunit carries glutamine (blue ball) in the channel pore region while R2 subunit carries a positively charged arginine residue (red ball). The electrostatic repulsion of positively charged arginine residue in the channel pore region prevents channel block by spermines.

To test for how the desensitization and rectification properties affect the overall receptor phenotype, we first carried out some pilot experiments. These experiments involved the cotransfection of two subunits of AMPA receptors being recombinantly expressed in human embryonic kidney (HEK) cells.

\subsection{Nondesensitizing phenotype penetrates more readily than desensitzing phenotype}

We cotransfected homomeric wild-type GluR2Q (abbreviated as 2Q) with nondesensitizing mutant GluR2Q or GluR1Q L504Y (abbreviated as 2Q(L-Y) and $1 \mathrm{Q}(\mathrm{L}-\mathrm{Y}))$ subunits at 7:1 ratio of DNA concentration. The response to a saturating pulse of glutamate was measured in the absence and presence of cyclothiazide. Due to the inclusion of nondesensitizing receptor subunits $(12.5 \%)$ we observed a small nondesensitizing component of the response in homomeric receptors as compared to heteromeric receptors (Figure 3A). For now we assume that mixing proper amounts of cDNA leads to proper protein expression (see control experiments in section 3.6). Outside-out patches were excised from HEK cells expressing these receptors and exposed to glutamate using fast application system (Rosenmund et al., 1998). 


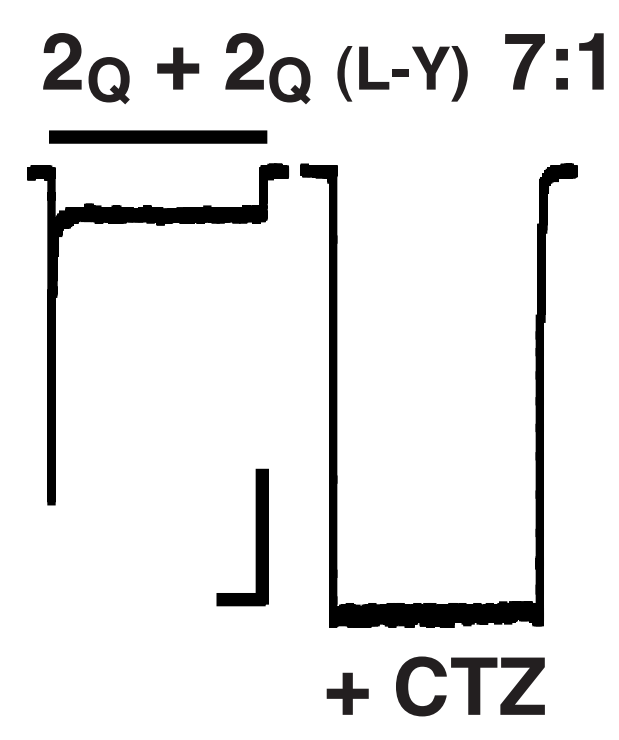

A

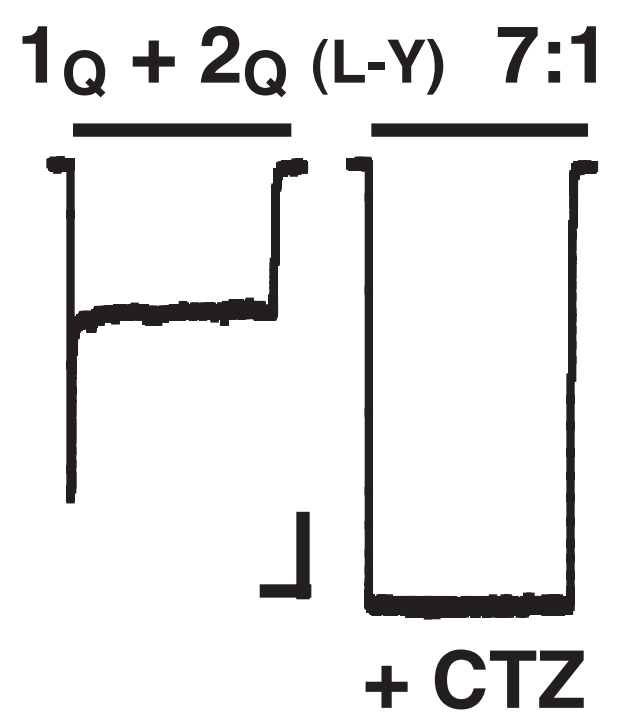

B

Figure 3. Nondesensitization component is different in homo and heteromers

(A) Typical current responses evoked by $10 \mathrm{mM}$ glutamate (black bar) in an outsideout patch excised from HEK cells cotransfected with 2Q $+2 \mathrm{Q}(\mathrm{L}-\mathrm{Y})$ at a ratio of 7:1 (homomeric combination). Application of saturating concentration of glutamate (10 $\mathrm{mM}$ ) results in current response that has steady state current of nearly $12 \%$ of the peak current. The experiment was repeated in the continuous presence of $100 \mu \mathrm{M}$ cyclothiazide $(+\mathrm{CTZ})$. Note that application of $100 \mu \mathrm{M}$ cyclothiazide blocks the desensitization completely of the assembled homomeric receptors (B) Current responses obtained by cotransfected $1 \mathrm{Q}+2 \mathrm{Q}(\mathrm{L}-\mathrm{Y}$ ) at a ratio of $7: 1$ (heteromeric combination). Note that the degree of nondesensitizing component of heteromeric receptors is larger than that for the homomeric receptors. Application of $100 \mu \mathrm{M}$ cyclothiazide blocks the remaining desensitizing component of the heteromeric coassembeled receptors. All scale bars are $200 \mathrm{~ms}, 50 \mathrm{pA}$

This result suggests that heteromeric AMPA receptors assemble more readily than homomeric receptors. This could be due to a difference in subunit assembly, or in the pattern of phenotype dominance. To investigate this difference between homoand heteromers further, we repeated the experiment using a second, independent marker.

\subsection{Nonrectifying phenotype penetrates more readily than rectifying phenotype}

A point mutation from glutamine to arginine (Q-R) was used as a second marker. We monitored the amount of inward rectification to assess the percentage of receptors that expressed the marker phenotype. Typical results obtained from 
homomeric combination of $2 \mathrm{Q}$ and $2 \mathrm{R}$ receptors or heteromeric combination of $1 \mathrm{Q}$ with $2 \mathrm{R}$ at a ratio of 1:1 in cotransfection experiments are shown in Figure 4A, 4B. The current amplitude was measured at $-30 \mathrm{mV}$ and $+30 \mathrm{mV}$ with $50 \mu \mathrm{M}$ spermine in the recording pipette and cyclothiazide in the extracellular solution. We measured rectification index in cotransfection experiments by considering the ratio of outward current to inward current (nonrectification index).

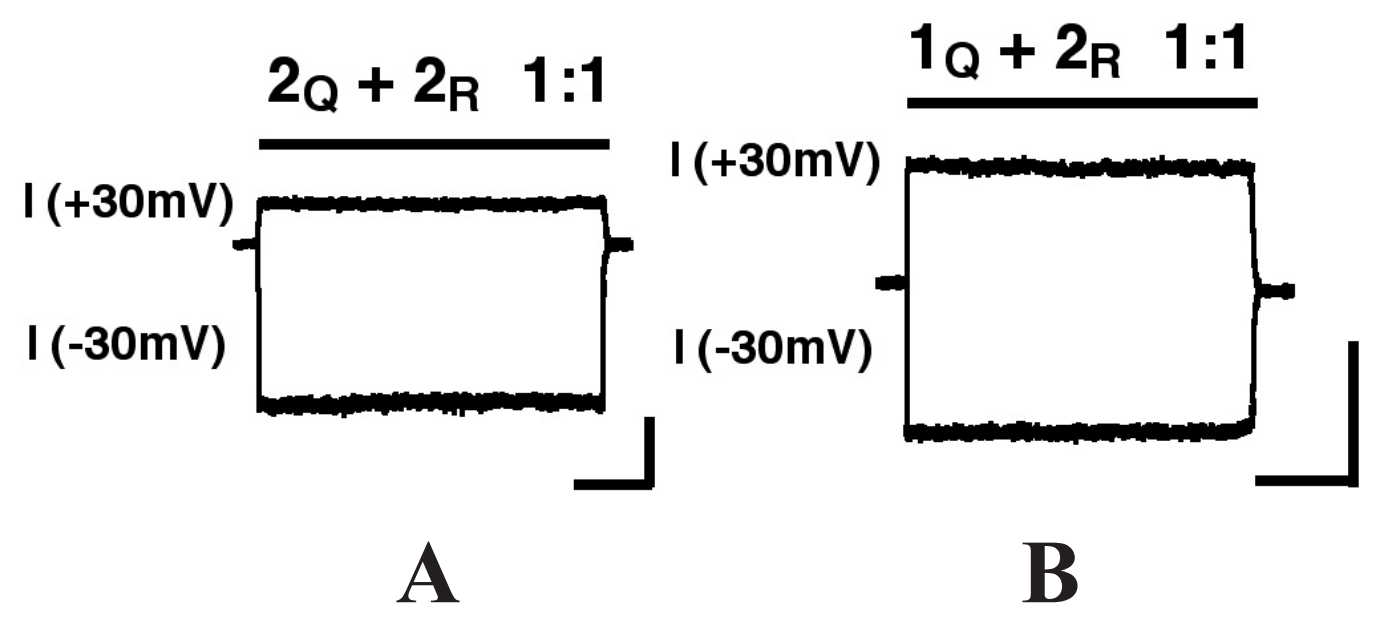

Figure 4. Rectification is different in homo and heteromeric AMPA receptors

(A) A typical current responses evoked by $10 \mathrm{mM}$ glutamate (black bar) in an outsideout patch excised from HEK cell cotransfected with $2 \mathrm{Q}+2 \mathrm{R}$ at a ratio of $1: 1$ (homomeric combination). Intracellular solution of the patch pipette contained $50 \mu \mathrm{M}$ spermines which block all the glutamine (Q) containing receptors at positive membrane potential $(+30 \mathrm{mV})$. The experiment was repeated in the continuous presence of $100 \mu \mathrm{M}$ cyclothiazide $(+\mathrm{CTZ})$ which blocks the desensitization completely. The current traces at $-30 \mathrm{mV}$ and $+30 \mathrm{mV}$ are overalaid on the top of each other. Note that the outward current at $+30 \mathrm{mV}$ is nearly $20 \%$ of the inward current (B) Current responses obtained by application of $10 \mathrm{mM}$ glutamate in an outside-out patch excised from an HEK cell cotransfected with $1 \mathrm{Q}+2 \mathrm{Q}(\mathrm{L}-\mathrm{Y})$ at a ratio of 1:1 (heteromeric combination). All the other experimental conditions were maintained same as mentioned for homomeric cotransfection experiments. Note that the outward current for heteromeric receptor combination is nearly $80 \%$ of the inward current. A larger amount of outward current in heteromeric cotransfection experiment versus homomeric cotransfection imply that the nonrectifying heteromeric receptors assemble more readily than that of homomeric receptors. All scale bars are $200 \mathrm{~ms}$, $50 \mathrm{pA}$. 
Surprizingly we observed that the nonrectification index was much larger for heteromeric coexpression $(0.80 \pm 0.03, \mathrm{n}=24)$ (Figure $4 \mathrm{~B})$ than for homomeric coexpression $(0.2 \pm 0.05, \mathrm{n}=12, \mathrm{p}<0.05)$ (Figure 4A). These results suggest that the nonrectifying heteromeric receptors assemble more readily than nonrectifying homomeric receptors. As the degree of rectification for heteromeric channels at a 1:1 subunit ratio was similar to results from AMPA receptors in principal neurons of hippocampus and neocortex, (Colquhoun et al., 1992; Geiger et al., 1995; Jonas et al., 1994) an expression of equal amounts of GluR2 subunit with GluR1/3/4 could explain the permeable properties of the channel in vivo.

Since both desensitization and rectification properties were different in homo and heteromeric subunit combination one may think of different mechanisms that control the homo and heteromeric assembly of AMPA receptors. However, we try to explain assembly of AMPA receptors using simple possible mechanisms.

\subsection{Plausible mechanisms for homo and heteromeric AMPA receptor assembly}

We considered two possible mechanisms of how homomers and heteromers can assemble in vitro based on the differences in the amount of nondesensitizing and nonrectifying components observed in homomeric and heteromeric receptor population. One possibility is that both homomeric and heteromeric receptors assemble in a random fashion but more of the heteromeric subunit configurations express the marker phenotype for desensitization or rectification properties (Figures $5 \mathrm{~A}$ and $5 \mathrm{~B})$.

A Random
Homomeric Assembly

\section{(X)}
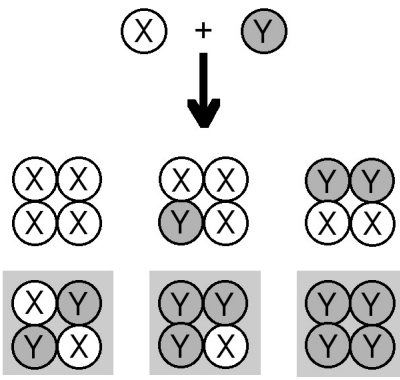

B Heteromeric Assembly
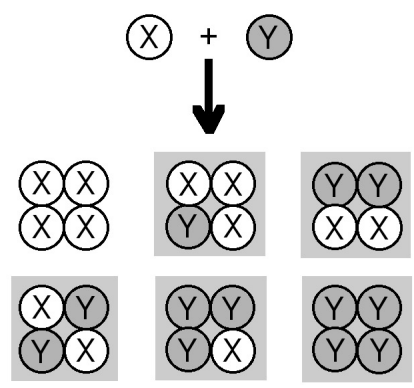

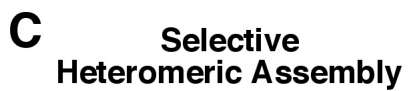

(X)
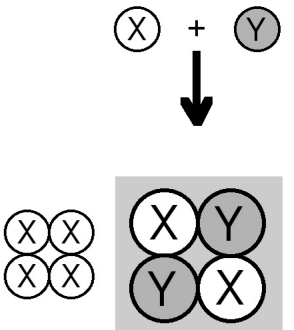

(Y)

Figure 5. Subunit configurations during assembly of homo- and heteromeric 
subunits of AMPA receptor.

(A) The six possible configurations for Wild-type (X) and marked (Y) homomeric subunits that assemble in a random fashion. In random homomeric assembly there are different possible ways of how marked and unmarked subunits can arrange within a tetrameric receptor configuration. Homomers can be either of marked type (all balls filled with $\mathrm{Y}$ ) or unmarked type (all balls filled with X). Heteromers can incorporate 1,2 , or 3 marked subunits in their configuration. 2:2 containing subunit configuration of marked and unmarked subunits can be arranged such that they can align either adjacent to each other or opposite to one another. Among these different configurations, only a subset of configuration expresses the marker phenotype. In this case, three out of six possible configurations expresses the marker phenotype (B) Random heteromeric assembly also results in six different possible configurations. If they assemble in a random fashion, then more subunit combinations have to express the marker phenotype. In this case five out of six configurations express the marker phenotype. This is shown by shaded box on five different subunit configurations (C) An alternative mechanism for heteromeric subunits that may preferentially assemble into a particular configuration with specific marker phenotype expression. In this case although only homomeric and 2:2 subunits containing configuration, only the latter configuration expresses the marker phenotype. This is shown by large shaded box marked on the 2:2 subunit configuration.

The mechanism for random assembly of heteromeric subunits is counterintuitive because it implies that, the marker phenotype can be changed by reversing an unmarked R2 subunit for an unmarked R1 subunit. This also requires that the substitution should affect both L-Y and Q-R phenotypes in a similar fashion. This explanation appears unlikely. Rather an alternative explanation could be that heteromeric subunits assemble in a preferential manner with a particular configuration that expresses both the L-Y and Q-R phenotypes (Figure 5C).

In lines with this hypothesis we next tested how the pattern of heteromeric phenotype expression is different from homomeric combination using a number of control experiments. These experiments were done to initially know whether channel expression correlates with the transfected DNA concentration.

\subsection{Protein expression is linear to the transfected $c D N A$ concentration}

We adopted two independent quantitative approaches to determine the level of functional subunit expression. In the first approach we estimated the relative expression levels of R1 and R2 subunit as homo and heteromers using biochemical techniques. The vectors we utilized for AMPA receptor coexpression differed only in 
the insert coding the receptor subunit. In most of the experiments the inserts differed only at a single amino acid residue. This difference is unlikely to yield a different level of receptor expression.

We tested protein expression as function of cDNA concentration by cotransfecting $\mathrm{R} 1(\mathrm{R})$ and $\mathrm{R} 2(\mathrm{Q})$ subunits at ratios 4:0, 1:3, 2:2, 3:1, 0:4. The total cDNA concentration was maintained at constant level. After harvesting the cells, expression of the cotransfected receptors were semiquantitatively examined in Western blots using specific antibodies against GluR1 and GluR2 subunits (Figure $6 A)$.
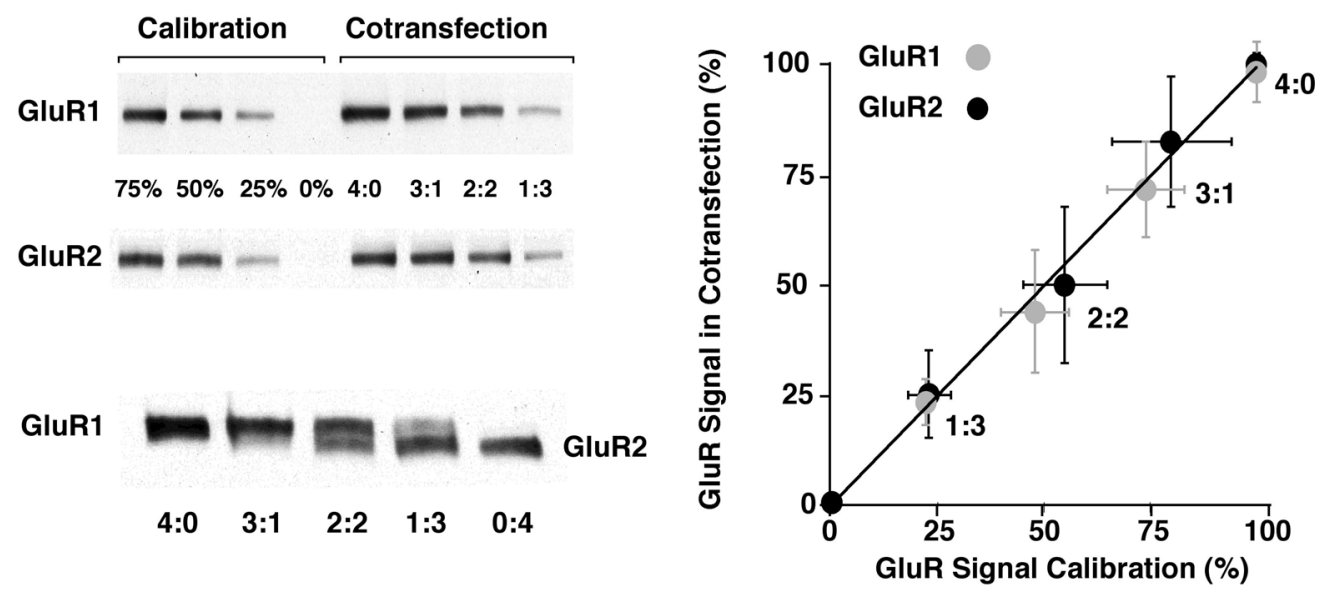

Figure 6. Biochemical assays to quantify protein expression in transfection

(A) The relative protein expression correlates with the amount of transfected plasmids. The plasmids encoding either for GluR1 or GluR2 subunit were transfected as homomers at cDNA ratio of 4:0 or $0: 4$. Heteromeric cotransfection experiment in HEK293 cells were carried out at different ratios (3:1, 2:2, 1:3). Various dilutions of 1Q or $2 \mathrm{R}$ subunit protein $(75 \%, 50 \%$, and $25 \%$ ) were loaded on a $6 \%$ SDS gel together with undiluted protein from the cotransfections for calibration purpose. We detected the blotted proteins by specific polyclonal antibodies raised against a specific subunit. The lower panel in figure 6A shows that the relative expression of both cotransfected subunits when the blot was incubated with specific antibodies raised against each subunit. In the paired bands, the upper band corresponds to GluR1 and the lower band to GluR2 subunits respectively (B) Densitometric quantification of signals from the GluR1- and GluR2-specific lanes. We plotted the signals from cotransfected GluR1 (gray circles) and GluR2 (black circles) against the corresponding calibration signals (three independent cotransfections). These signals were normalized to the undiluted homomeric transfection of GluR1 and GluR2 subunits. To assess systematic errors in the densitometric analysis two undiluted lanes were run. Densitometric analysis also showed that the subunit expression of GluR1 and GluR2 subunit varies linearly with cDNA concentration. These biochemical 
assays were performed by Dr. Ralph Nehring.

Based on densitometric analysis we confirmed that the subunit expression correlates well with the amount of DNA used in the transfection (Figure 6B).

\subsection{Quantification of functional receptor expression}

The biochemical in vitro assays performed in this study does not distinguish the receptors expressed on the surface of the membrane and intracellular compartments. Previous studies on cell surface expression have also shown that there is no significant difference in the efficiency of the receptor insertion for GluR1 and GluR2 subunit into membrane (Hall et al., 1997).

We adopted electrophysiological approach to determine the number of functional channels expressed following the transfection of cDNA. This is based on the detection of an approximately constant number of channels from patch to patch measurements. We quantified the number of ion channels in outside-out patch experiments. HEK cells were transfected with R1(Q) or R2(Q). In every case, the total quantity of DNA was maintained constant in the transfection. Cyclothiazide was added to the control solution and the glutamate-evoked currents were recorded from a total of 471 patches. The number of channels in a patch was estimated from the steady state current. The single channel current and the open probability measurements varied considerably patch to patch. The average number of channels per patch was very similar for the two different homomers (R1 (Q): $677 \pm 77$ channel/patch; R2 (Q): $718 \pm 62$, Figure 7). Based on these results we can assume that receptor subunit expression in our cotransfection experiments is highly correlated with the DNA concentrations used.

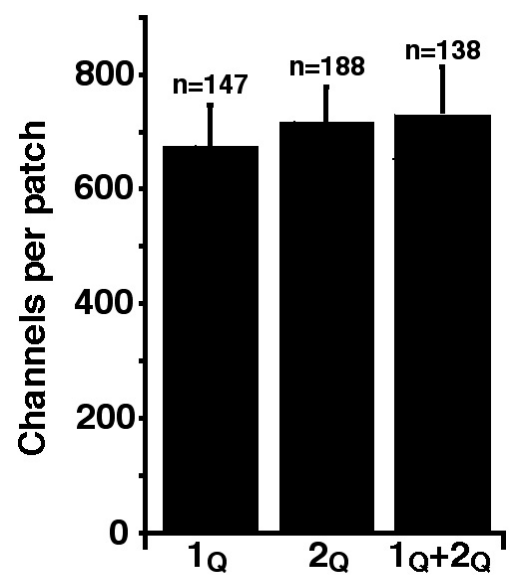


Figure 7. Quantifying the number of channels in outside out patch measurements

The bar graph represents the average number of channels per outside-out membrane patch from HEK cells transfected with R1Q or with R2Q or cotransfected with R1Q+ R2Q (1:1 ratio). This was calculated by considering the ratio of the current (in 100 $\mu \mathrm{M}$ cyclothiazide) and average single channel current of individual subunit. The number of channels per patch is not significantly different in homo (1Q or 2Q) and heteromeric receptors $(1 \mathrm{Q}+2 \mathrm{Q})$.

These results suggest that the number of glutamate receptor subunits that are available for assembling into functional channels most likely vary linearly with cDNA concentration used in transfection experiments.

\subsection{Quantification of single channel properties of homo and heteromeric AMPA receptors}

The semiquantitative biochemical approaches in conjunction with the number of channels per patch being determined by electrophysiological approach provided us the initial steps to understand the properties of homo and heteromeric combination of receptors. We furthermore tried to model the behavior of a population of receptor assemblies by relying on assumptions about the single channel conductance of homomeric and heteromeric channels. First, we performed the single channel recordings from cells expressing either homomeric (R1 or R2 subunits) or heteromeric combination (R1 and R2) of receptors. Secondly we determined the time averaged single channel currents and conductance parameters from mean-variance analysis for homo and heteromeric combination of these subunits. These parameters are essential for constructing the phenotype model for assembly of AMPA receptors. This allowed us to assign specific behavior for specific assembly in phenotype model.

We excised patches containing a single channel as previously described (Rosenmund et al., 1998). All single channel recordings were made at $-80 \mathrm{mV}$ in the presence of a saturating concentration of glutamate $(10 \mathrm{mM})$ and cyclothiazide (100$\mu \mathrm{M})$. We measured the average steady-state current directly in outside-out patch measurements. The average steady state current is the product of single channel current and open probability (see section 2.7.3, equation (1)). The average steady state current represents the summation of current from all subconductance states (Rosenmund et al., 1998; Smith et al., 2000; Yamada and Tang, 1993). The ratio of the average steady-state current to number of channels present per patch were 
considered for further analysis. We measured single channel current from heteromeric channels by cotransfecting $1 Q+2 Q / R$ at ratio of $1: 1$ concentration of cDNA (Figure 8A).

A

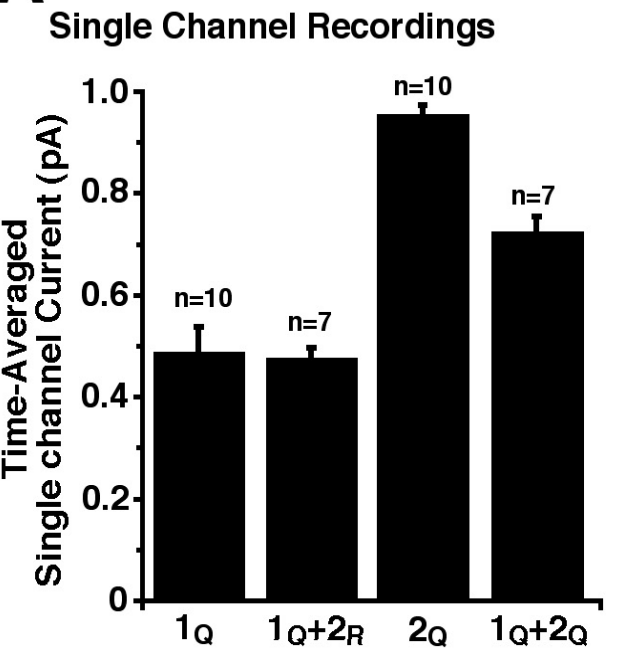

B

\section{Noise Analysis}

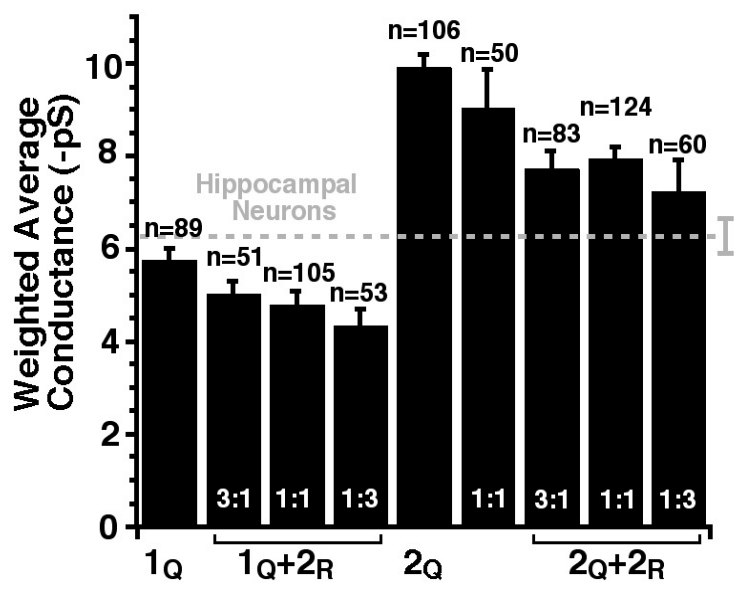

Figure 8. Deriving the conductance parameters from single channel recordings and noise analysis

(A) Summary of the results obtained from single channel experiments. Outside out patches containing a single channel were pulled from HEK cells transfected with $\mathrm{R} 1 \mathrm{Q}$, or with $\mathrm{R} 2 \mathrm{Q}$, or cotransfected with $\mathrm{R} 1 \mathrm{Q}+\mathrm{R} 2 \mathrm{Q}$ or $\mathrm{R} 1 \mathrm{Q}+\mathrm{R} 2 \mathrm{R}$ at a ratio of $1: 1$. The bar plot shows the time-averaged single channel current for homomeric and heteromeric subunit combination. n corresponds to number of outside-out patches analysed for measuring time-averaged single channel current (B) Summary of the results obtained from multichannel experiments. Weighted average single channel conductance was estimated by using noise analysis for various subunit combinations. Both homomeric and heteromeric combination of receptors were used for noise analysis experiments. Experiments were performed on 10-24 patches from each subunit combination $(1 \mathrm{Q}, 2 \mathrm{Q}, 1 \mathrm{Q}+2 \mathrm{R}$ and $2 \mathrm{Q}+2 \mathrm{R})$. Different ratio $(1: 1,3: 1,1: 3)$ of cDNA concentrations were tested for homomeric $(2 \mathrm{Q}+2 \mathrm{R})$ and heteromeric combination $(1 \mathrm{Q}+2 \mathrm{R})$ experiments. The dotted line represents the single channel conductance of outside out patches excised from native AMPA channels of hippocampal neurons. The weighted average conductances of homomeric 2Q or $2 \mathrm{Q}+2 \mathrm{R}$ combination were nearly two fold larger than that from homomeric1Q or heteromeric $1 \mathrm{Q}+2 \mathrm{R}$ receptors. $\mathrm{n}$ represents the number of traces used for the fluctuation analysis.

From single channel results of heteromeric cotransfection, we observed that the coassembled receptors were dominated by those heteromeric channels that coassembled with a preferred subunit configuration. Contrary to this, homomeric 
cotransfections produced many different subunit configurations. We required a much larger number of single channel recordings to adequately sample the mixed population. To overcome these difficulties we recorded currents from multi-channel patches and applied steady-state mean-variance noise analysis (see section 2.7.3). Mean-variance noise analysis estimates the weighted average single channel conductance for a mixed population of receptors (Neher and Stevens, 1977; Traynelis et al., 1993). The requirements for noise analysis include that open probability for the channel is $<30 \%$. We performed these experiments in the absence of cyclothiazide. With this technique we were able to efficiently analyze a range of different subunit combinations (Figure $8 \mathrm{~B}$ ). We observed that the pattern of average conductances for R1Q, R2Q homomers and R1Q+R2 (Q/R) heteromers (Figure 8B) were parallel to the pattern of average single channel currents (Figure 8A). We confirmed this by actual single channel recording experiments. Heteromeric cotransfection experiments of R1Q with R2R yield conductance levels of about $5 \mathrm{pS}$. This conductance value was similar to results that we obtained from hippocampal neurons $(6.3 \pm 0.4 \mathrm{pS}, \mathrm{n}=26$, Figure $8 \mathrm{~B}$ ). We did not observe a significant decrement in the single channel conductivity by increasing the amounts of R2(R) subunit. These results are consistent with the assumption that assemblies containing three subunits of R2(R) subtype have low conductance similar to homomeric R2(R) subunit (Swanson et al., 1997b). These experimental results were used as a guide when selecting single channel current parameters for constructing the phenotype expression models (see below).

\subsection{Analytical procedures for classification of specific phenotypes in cotransfection experiment}

Depending on specific combination of subunits carrying marker for either desensitization or rectification, we now wanted to determine if there exists a clean switch from desensitizing to non-desensitizing and rectifying to non-rectifying channel phenotype. Furthermore, we developed protocols that allowed us to determine the existence of any intermediate phenotypes possessing partial desensitization or partial rectification properties. 


\subsubsection{Quantification of desensitizing and nondesensitizing population of receptors}

When two subunits carrying either desensitizing or non-desensitizing phenotype are cotransfected, one can expect that the resulting population of coassembled receptors may have mixed population of receptors either bearing desensitizing or non-desensitizing characteristics. To classify desensitizing and nondesensitizing receptors from mixed population we first cotransfected wild-type GluR1 and mutant GluR2 L504Y at 11:1 ratio of DNA concentration.

\section{Glutamate $10 \mathrm{mM}$}

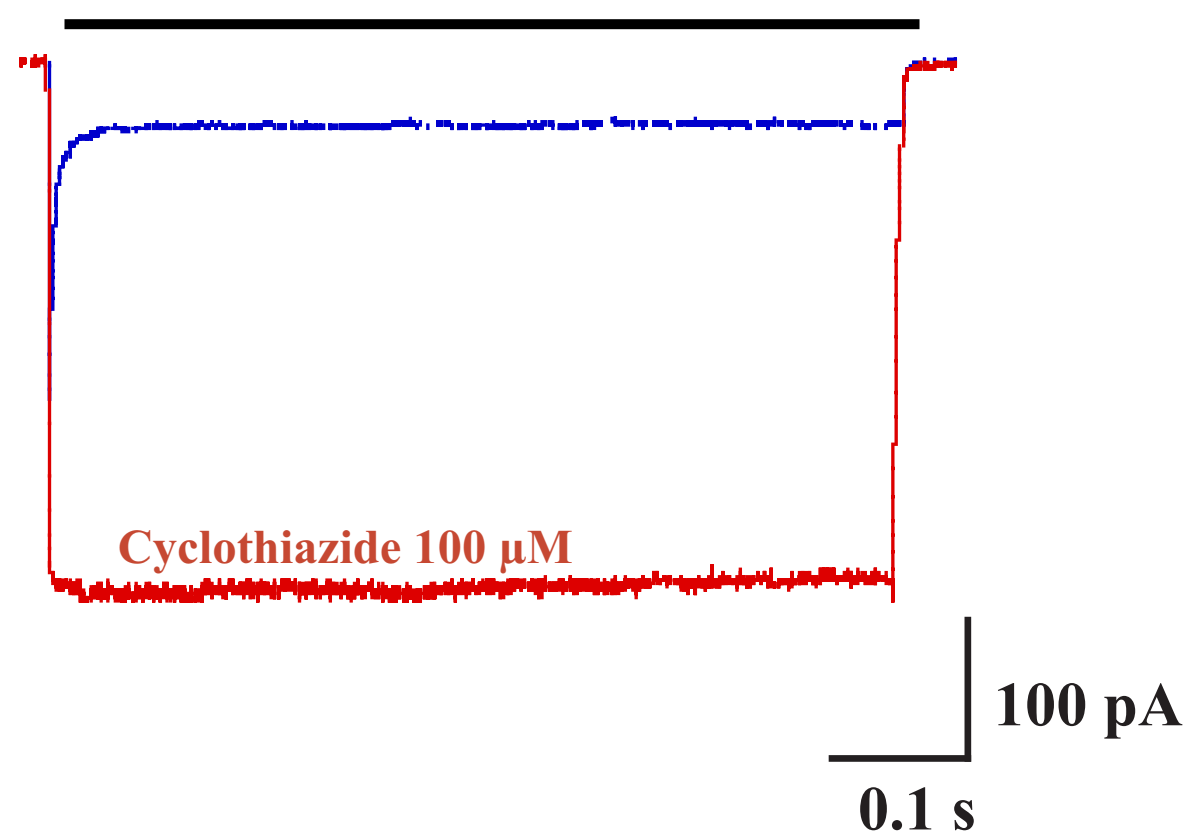

Figure 9. Quantification of desensitizing and nondesensitizing population of receptors

Exemplary glutamate (10 mM, black bar) induced current traces from an outside-out patch excised from a HEK293 cell cotransfected with R1+R2Q(L504Y) at 11:1 in cotransfection experiments in absence (blue trace) and presence of cyclothiazide (100$\mu \mathrm{M}$, red trace). Preincubation of cyclothiazide $(100 \mu \mathrm{M})$ in the presence of saturating concentration of glutamate $(10 \mathrm{mM})$ led to complete block of desensitization. These current traces recorded at $-30 \mathrm{mV}$ had a desensitizing current component and a steady state component. The steady state component corresponds to non-desensitizing population of receptors in cotransfection. 
We measured the total receptor population by preincubating the patches with cyclothiazide. Cyclothiazide $(100 \mu \mathrm{M})$ blocks the desensitization of wild-type receptors while leaving nondesensitizing responses unaffected (Stern-Bach et al., 1998). The ratio of steady-state responses in the absence and presence of cyclothiazide was used to quantify the fraction of receptors expressing the nondesensitizing marker phenotype. This quotient represents the index for nondesensitization.

\subsubsection{No evidence for intermediate desensitization phenotype in cotransfection experiments}

When homomeric R1 and R2 subunit or their mutants (L-Y) combine, individual receptor assembly may carry phenotypes of one of these receptors. In contrast, one may expect some population of the receptors to possess intermediate characteristics of desensitizing and nondesensitizing nature. We developed a protocol that allows us to test for possible intermediate forms of desensitization. We used the exponential fits for the desensitization phase as a measure for determining the intermediate phenotypes. A single exponential fit corresponds to the presence of one type of receptor population (either, desensitizing or non-desensitizing), while double exponential fit corresponds to the presence of intermediate phenotypes. We observed that the desensitization phase could not be well fit by a single exponential in some patches of cotransfection experiments (wild-type and L-Y mutant receptors combinations). The analysis suggested that an intermediate desensitizing component with a time constant of $13-70 \mathrm{~ms}$ was present. However, we found it difficult to quantify this component, as both wild-type $\mathrm{R} 1(\mathrm{Q})$ receptors and their mutant homologue exhibited a small fraction of desensitization in the same time constant range. A double exponential fit to the homomeric $\mathrm{R} 1(\mathrm{Q})$ response revealed a dominant fast component and a smaller component with the time constant of $14 \pm 1.5$ ms $(n=19) \quad$ (amplitude $16 \pm 3 \%$ of the peak response) (Fig 10 A, B). Homomeric R1(Q)L-Y desensitized weakly to $85 \pm 1.5 \%$ of the peak response with a time constant of $51 \pm 10 \mathrm{~ms}(\mathrm{n}=17)$. Similar values were obtained from R2(Q) and its mutant analogue. These weak components of desensitization in homomers make it difficult to unambiguously detect an intermediate component in GluR(wild-type) /GluR L-Y cotransfections.

In fact, in cotransfection experiments of either homomeric combination of 
GluR2Q with GluR2R L-Y or in heteromeric combination of GluR1Q with GluR2R L-Y, we did observe a small increase $(<10 \%)$ in the relative amplitude of the intermediate component when the fraction of nondesensitizing receptors in cotransfection experiment was $20-30 \%$. However we did not observe that the results follow a clear trend as the cDNA ratio was varied (Figure 9B). The presence of a small intermediate component however did not affect our results, as we did not try to distinguish the sub-populations between desensitizing receptor population. Instead we simply compared the steady state responses in the absence (represents the fraction of nondesensitizing receptors) and the presence of cyclothiazide (represents the total population of receptors) as a measure for desensitizing and non-desensitizing receptor populations.
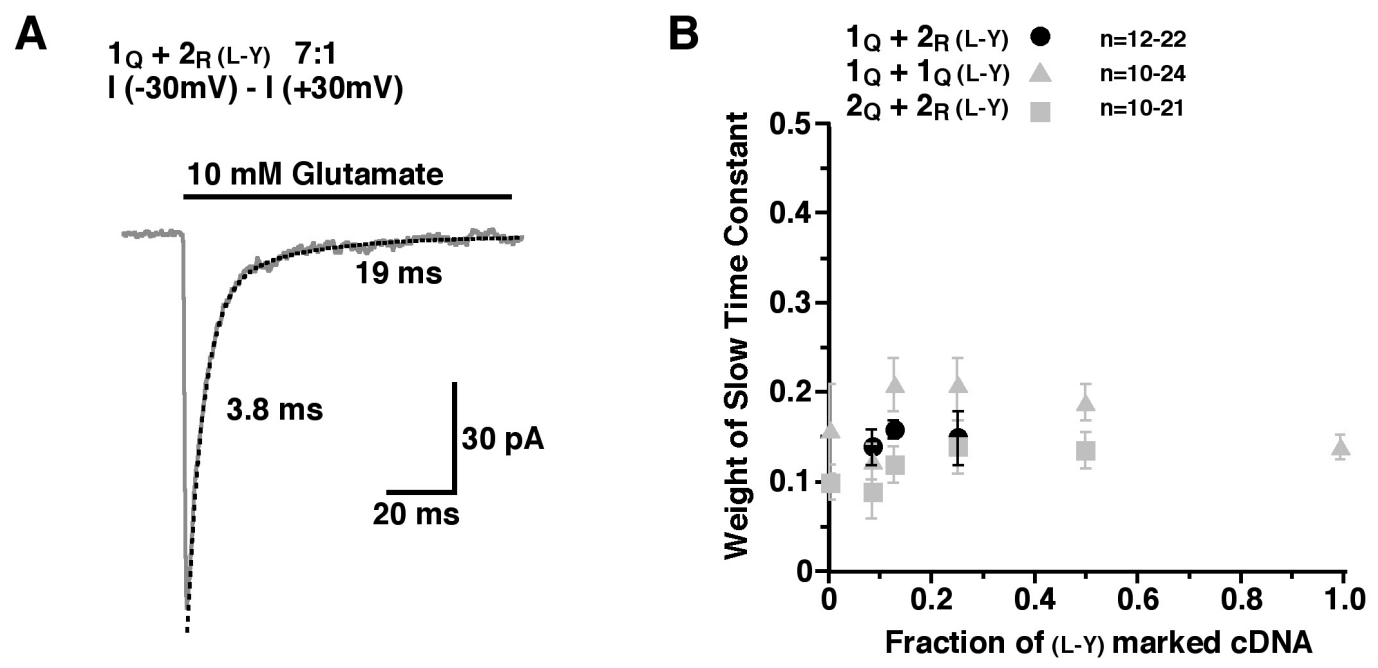

Figure 10. Absence of intermediate phenotype for desensitization properties in cotransfection experiments

(A) A raw trace representing the desensitizing component of the current from an outside-out patch containing cotransfected $1 \mathrm{Q}+2 \mathrm{R}(\mathrm{L}-\mathrm{Y})$ subunits at a ratio of $7: 1$. The current at $+30 \mathrm{mV}$ was subtracted from the current at $-30 \mathrm{mV}$ to remove the steady-state component due to nondesensitizing receptors. The current trace was fit with a double-exponential function (dashed line) (B) The graph describes the analysis of the time constants of desensitization. The relative amplitude of the slow component of desensitization time constant was plotted against the fraction of mutant cDNA concentration for various subunit combinations. The slow component did not systematically increase with the increasing fraction of subunits carrying the L-Y marker. This suggests that receptor assemblies do not exhibit intermediate desensitization properties. 


\subsubsection{Quantification of rectifying and nonrectifying population of receptors in cotranfection experiments}

As a second approach to determine the different properties of coassembled receptors in cotransfection experiments, we employed the rectifying and nonrectifying behavior of individual subunits. When two subunits carrying either of these phenotype are cotransfected, one may expect that the resulting population of coassembled receptors may have mixed population either bearing channels of rectifying or nonrectifying characteristics. To classify them from mixed population R1 and R2 subunits were first marked to carry either glutamine (Q) or arginine (R) in the channel pore region. An arginine residue in the channel pore region conduct ions linearly (nonrectifying) while the presence of glutamine residue makes a channel to adopt inwardly rectifying properties due to the presence of intracellular spermines.
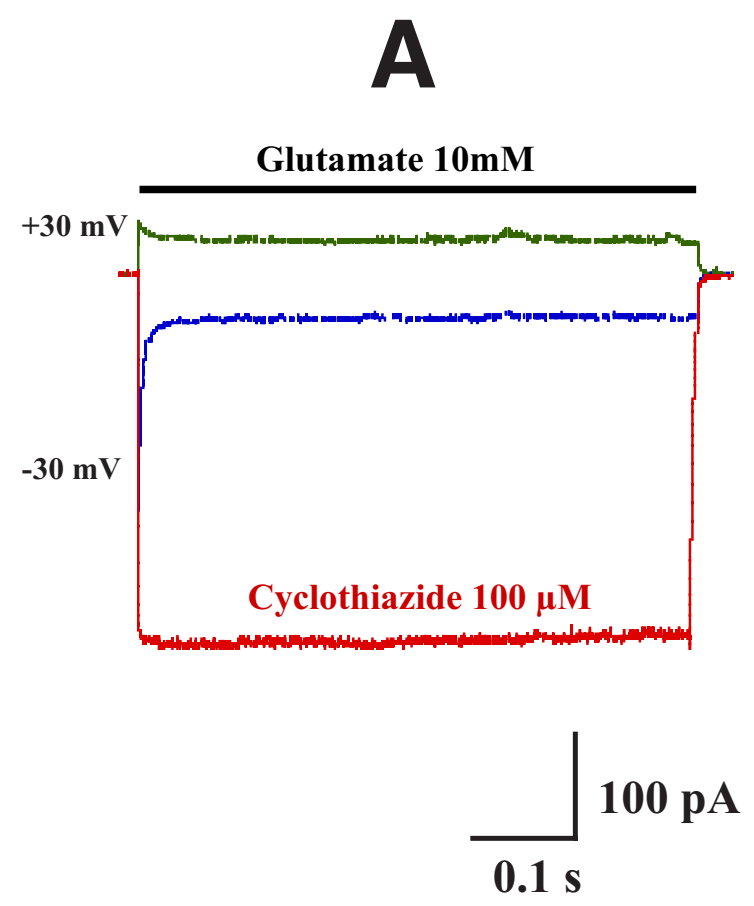
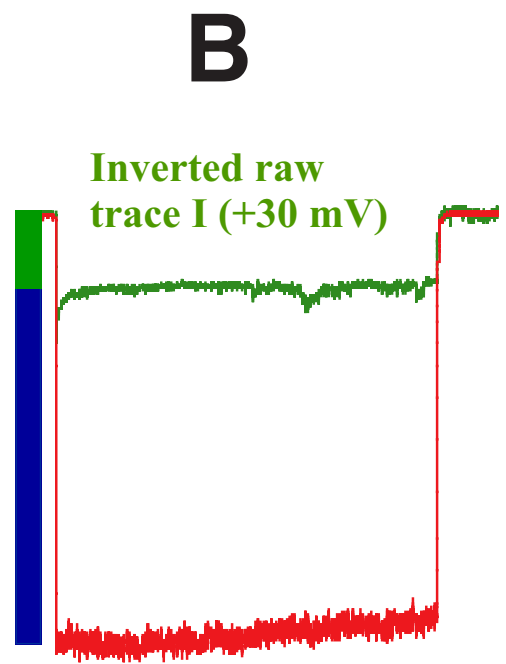

Raw trace I (-30 mV) + Cyclothiazide $150 \mathrm{pA}$

Figure 11. Quantification of rectifying and nonrectifying population of receptors

(A) A representative trace from an outside out patch expressing R1(Q) and R2(R) L-Y showing the loss of rectification at positive membrane potential $(+30 \mathrm{mV})$ in the presence of $50 \mu \mathrm{M}$ intracellular spermines. Intracellular spermines block all the homomeric $\mathrm{R} 1(\mathrm{Q})$ receptors at positive membrane potential. Outward currents at +30 $\mathrm{mV}$ hence will be induced by only those receptors that possess $\mathrm{R} 2(\mathrm{R})$ subunits. This 
outward current was completely nondesensitizing, nonrectifying and corresponds to the nonrectifying fraction of the receptor population. Note that the amount of steady state component at $-30 \mathrm{mV}$ is equal to the steady-state component at $+30 \mathrm{mV}$. The outward current induced by nonrectifying $\mathrm{R} 2(\mathrm{R}) \mathrm{L}-\mathrm{Y}$ containing receptors were not potentiated by cyclothiazide at $+30 \mathrm{mV}$. Orange trace and green trace corresponds to current at positive membrane potential $(+30 \mathrm{mV})$ in the presence and absence of $100 \mu \mathrm{M}$ cyclothiazide. (B) The rectifying and nonrectifying channels were quantified from the total population of receptors by subtracting the inverted current trace at $+30 \mathrm{mV}$ from the current trace at $-30 \mathrm{mV}$ in the presence of cyclothiazide. The inward current in the presence of cyclothiazide represents total population of rectifying and nonrectifying channels that are formed in cotransfection experiments. In the presence of $50 \mu \mathrm{M}$ spermines, the outward current represents the current that flows exclusively through those receptors that possess arginine in the channel pore region (R2(R)), while all channels possessing glutamine residue $(\mathrm{R}(1 \mathrm{Q}))$ in channel pore will be completely blocked.

We quantified the amount of rectification by measuring the responses both at negative $(-30 \mathrm{mV})$ and positive membrane potential $(+30 \mathrm{mV})$ in the presence of spermines $(50 \mu \mathrm{M})$ (Figure 11A). This allowed us to distinguish the linear versus rectifiying channels in coassembled receptors. In cotransfection experiments we expressed inwardly rectifying $\mathrm{R} 1(\mathrm{Q})$ subunit with linearly conducting $\mathrm{R} 2(\mathrm{R}) \mathrm{L}-\mathrm{Y}$ at 11:1 ratio of DNA concentration. Spermine served as pharmacological probe to block the current responses of glutamine carrying subunits $\mathrm{R} 1(\mathrm{Q})$ in the mixed population at positive membrane potential $(+30 \mathrm{mV}$ ) (Bowie and Mayer, 1995; Donevan and Rogawski, 1995). Responses at $+30 \mathrm{mV}$ are thus produced almost entirely by linearly conducting receptors. By subtracting steady state current at $+30 \mathrm{mV}$ from the responses at $-30 \mathrm{mV}$ in the presence of $\mathrm{CTZ}$ we were able to pool the fraction of linear versus rectifying channels in the mixed population. The ratio of steady state current amplitude at $+30 \mathrm{mV}$ and $-30 \mathrm{mV}$ in the presence of $100 \mu \mathrm{M}$ cyclothiazide represents the rectification index $(\mathrm{I}+30 \mathrm{mV} / \mathrm{I}-30 \mathrm{mV})$.

\subsubsection{No evidence of intermediate phenotype for rectification properties in cotransfection experiments}

Our initial experimental approaches failed to observe any intermediate phenotype of desensitization in mixed population of receptors. We furthermore quantified if any intermediate phenotype of rectification properties exists in cotransfection experiments of rectifying channels with nonrectifying ones. A shift from glutamine and arginine residues of the channel pore region on either R1 or R2 
subunit did not affect the rectification index $(\mathrm{I}+30 \mathrm{mV} / \mathrm{I}-30 \mathrm{mV} \sim 1)$ to a significant extent. Furthermore, the rectification index did not change by cotransfecting subunits carrying markers for both desensitization (L-Y) and rectification (Q-R) on either R1 or R2 subunits (Figure 12B) at various concentration of DNA in cotransfection experiment.

We observed in additional set of experiment that the desensitizing component was absent at positive membrane potentials when the linearization (non-rectifying) and nondesensitizing markers were present on either the same subunit or on different subunits (Figure $12 \mathrm{~A})$. This was clear by applying cyclothiazide $( \pm 100 \mu \mathrm{M}$ CTZ) which failed to show any potentiation of the outward current. The amplitude ratio was invariant across all mutant receptor fractions tested. We can conclude that the receptors exhibiting the non-desensitizing phenotype also exhibited the linear conduction phenotype implying that no significant intermediate inwardly rectifying current component exists (Figure 12B) (see Washburn et al., 1997).

A

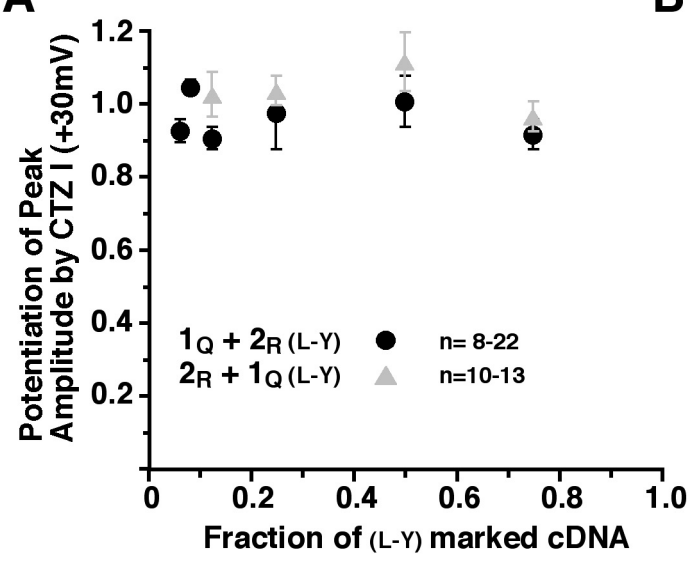

B

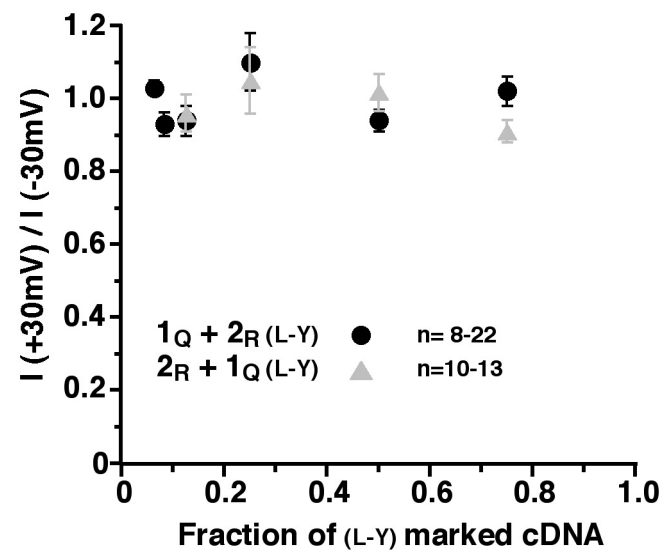

Figure 12. Evidence for the absence of intermediate desensitizing phenotype in cotransfections.

(A) The plot describes the absence of peak amplitude potentiation by cyclothiazide at positive potential $(+30 \mathrm{mV})$ for heteromeric cotransfection experiments. Data corresponds to cotransfection of $1 \mathrm{Q}+2 \mathrm{R}(\mathrm{L}-\mathrm{Y})$ where the markers for linearization (nonrectification) and nondesensitization are present on the same subunit (1Q $+2 \mathrm{R}(\mathrm{L}$ -Y) (black circles) or on different subunits $(2 \mathrm{R}+1 \mathrm{Q}(\mathrm{L}-\mathrm{Y})$ (gray triangles). Application of $100 \mu \mathrm{M}$ cyclothiazide did not lead to any significant potentiation of the outward current at various ratios tested in cotransfection experiement (B) Amplitude ratio of steady-state currents at $+30 \mathrm{mV} /-30 \mathrm{mV}$ in heteromeric cotransfections of $1 \mathrm{Q}+2 \mathrm{R}(\mathrm{L}$ -Y) (black circles) and $2 \mathrm{R}+1 \mathrm{Q}(\mathrm{L}-\mathrm{Y})$ (gray triangles). The amplitude ratio was invariant across all mutant receptor fractions tested. 
Thus employing different markers for either desensitization or rectification we confirmed that the population of assembled receptors behave purely either desensitizing or nondesensitizing and rectifying or nonrectifying without existing in any other intermediate forms. Based on the classification of (a) desensitizing from nondesensitizing phenotype, (b) rectifying from nonrectifying channels we built a quantitative model for AMPA receptor assembly. The model predictions are tested in various cotransfection experiments with both the markers for desensitization and rectification. These models are essential to understand whether subunits assemble in stochastic or preferential manner.

\subsection{A quantitative model of subunit assembly and phenotype dominance}

The assembly of AMPA receptors is, by definition a complex combinatorial process. Our work represents a rigorous attempt to investigate and model this process. This section emphasizes on the quantitative description underlying the mechanisms involved in assembly process. We present several simple cartoons to describe this process that allows us to assign the specific functions for specific assemblies.

\subsubsection{Binomial model for homomeric subunit assembly}

We distinguished the hypotheses of random and preferential assembly mechanism in homo and heteromeric coassembly of AMPA receptor subunits by developing quantitative models. Two models were developed for AMPA receptor assembly and phenotype dominance. One of the models was based on random subunit assembly of AMPA receptors and the other on selective assembly mechanisms. We utilized these models to investigate (a) the difference between phenotype expression of homomeric and heteromeric combination of subunits and (b) the specific homomeric subunit configuration that expresses the marker phenotypes.

AMPA receptors are thought to be tetrameric in nature (Mano and Teichberg, 1998; Rosenmund et al., 1998). The subunits thus can have six possible spatial configurations (Figure 13). As marked (Y) and unmarked subunits (X) have an almost identical structure, homomeric receptors are assumed to assemble in a stochastic fashion. 


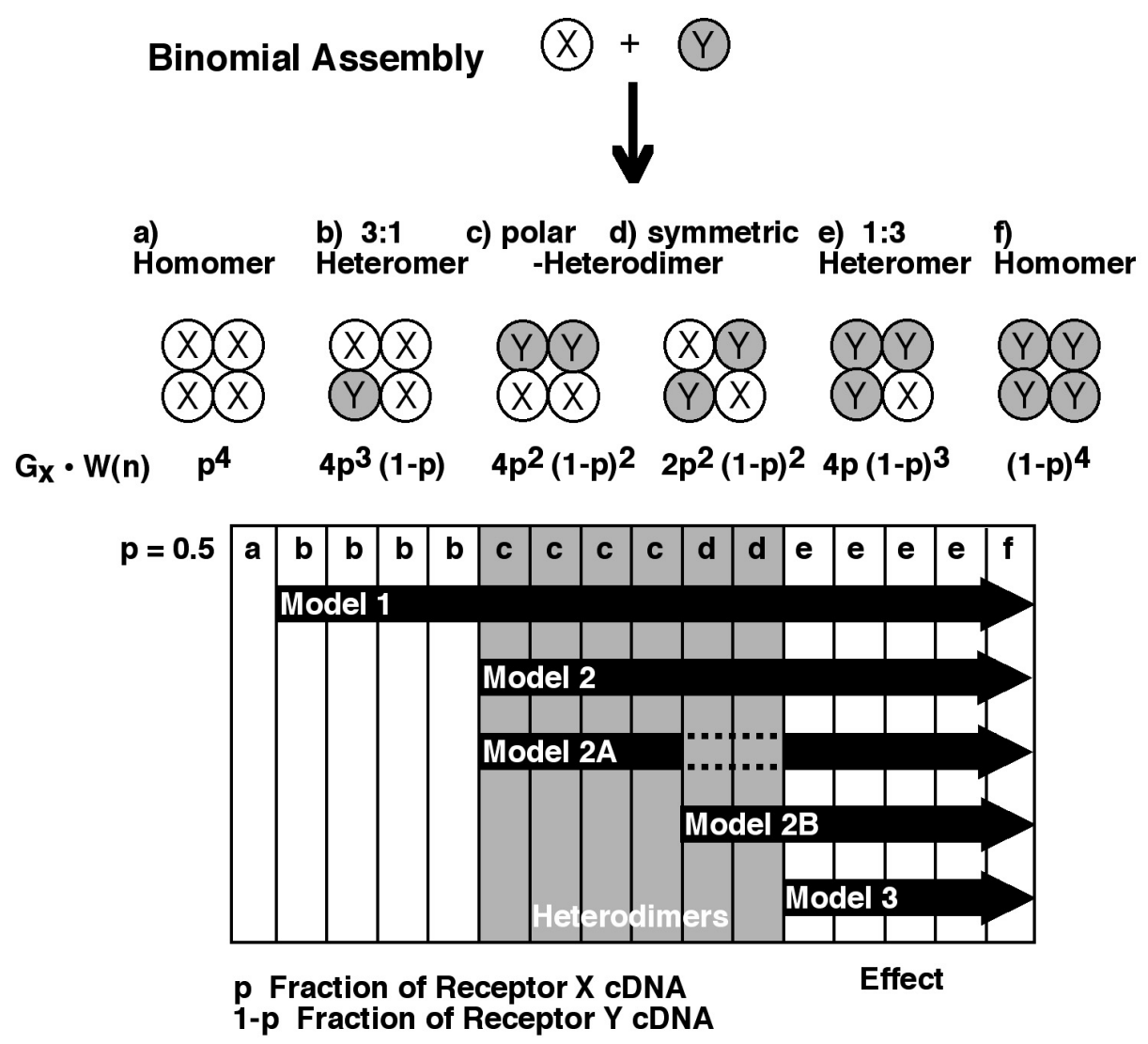

Figure 13. Phenotype expression models

The cartoon represents the binomial assembly model when two different subunits, $\mathrm{X}$ and $\mathrm{Y}$, assemble in a stochastic fashion. In such stochastic assembly the frequency of the various subunit combinations follows a binomial distribution. Assuming identical concentration of receptor $\mathrm{X}$ and $\mathrm{Y}(\mathrm{p}=0.5)$, there are 16 possible tetrameric assemblies, shown schematically as 16 vertical columns, but there are only six distinct subunit configurations $(\mathrm{a}-\mathrm{f})$. Subunit $\mathrm{Y}$ (shaded) carries a marker mutation for either desensitization or rectification. An arrow (model 1-3) indicates the subunit configurations that express the marker phenotype in each model. For example, Model 1 assumes that a single subunit of receptor $\mathrm{Y}$ is sufficient to dominate the receptor assembly. The 6 out of 16 possible heterodimers are marked in grey. Note that the two forms of heterodimers are distinguished and furthermore point symmetric dimers $(2$ of 6) have a twofold lower probability than the polar form (4 of 6). The 2X: $2 \mathrm{Y}$ heteromeric assemblies ( $c$ and $d$ ) are shown in gray color. A polar and a point symmetric form are represented as shown in (c) and (d). W (n) represents the distribution function and Gx corresponds to the conductance of individual subunit configuration.

We used binomial distribution to calculate the percentage of receptors that will assemble into each of the six configurations at any given receptor DNA fraction, $p$ and (1-p) using the distribution function $W(n)=\sum p^{n}(1-p){ }^{n-1}$ (Figure 13) as well as 
specific distribution of assemblies when both receptors are present in equal concentration $(p=0.5)$. At equal concentration of the two subunits, only $1 / 16$ of the assemblies are homomeric for each receptor, while $6 / 16$ of the assemblies are heterodimers. 2 of the 6 heterodimers are assembled with specific point symmetry, while twice as many are polar symmetric heterodimers.

Based on location of identical subunits one can classify these heterodimers into two subclasses. The point symmetric and polar heterodimers. Point symmetric heterodimers (Figure13-(d)) allow identical subunits to be positioned on opposite side of the channel pore region while polar heterodimers allow identical subunits to be positioned side-by-side (Figure 13-(c)).

\subsubsection{Selective assembly model for heteromeric receptors}

The assembly of heteromeric receptors in contrast to homomeric receptors may not be random. Rather the assembly of R1 and R2 subunits might be mediated by interactions that may favor a particular subunit configuration. In selective assembly mechanism we have chosen a simple model where only homomers and point symmetric subunit configurations are assembled. Even with this simplest model, one can obtain binomial distribution of the three permitted subunit configurations (Figure 14). Assuming that each subunit configuration expresses either the wild-type or the marker phenotype without any intermediate phenotypes, one can utilize the assembly models to predict the total fraction of channels that express the marker phenotype (desensitization and rectification). 


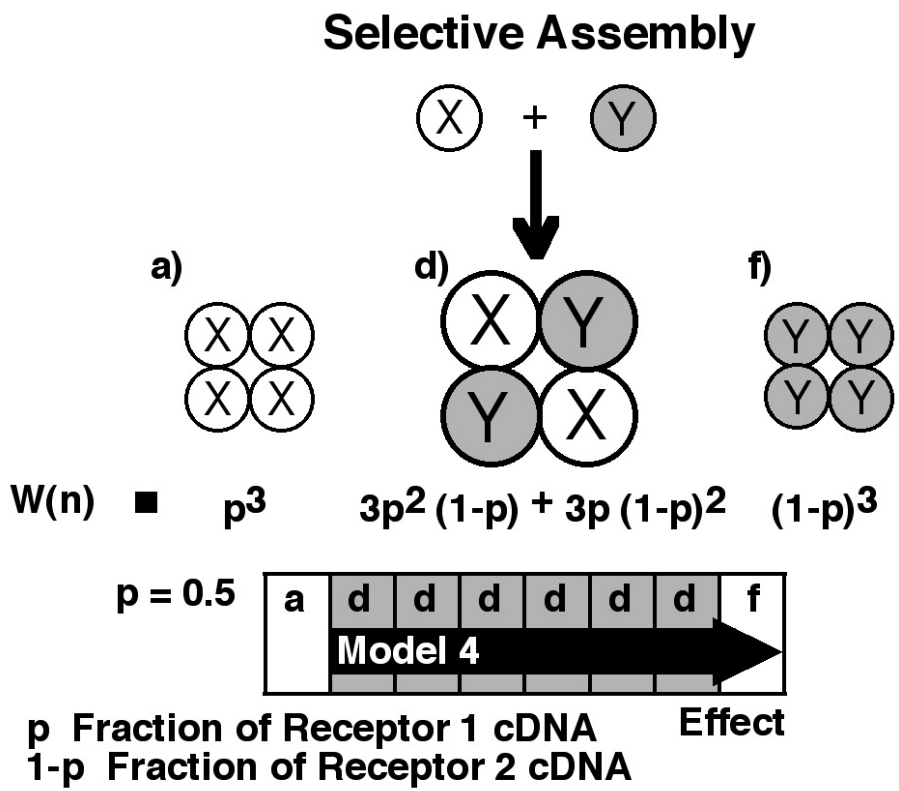

Figure 14. Selective assembly of AMPA receptor subunits

The cartoon represents the selective assembly model when two different subunits, $\mathrm{X}$ and $\mathrm{Y}$, assemble in a preferential fashion. In selective assembly model, the interactions between marked $(\mathrm{Y})$ and unmarked $(\mathrm{X})$ subunits may destabilize asymmetric subunit configurations. In such a case, a binomial assembly model predicts the frequency of the three symmetric configurations. Shown below the subunit configuration is the phenotype expression Model 4. This model assumes that two marked subunit are sufficient to alter the phenotype of the receptor.

\subsubsection{Model assumptions}

A series of phenotype expression models were developed each of which assigns the marker phenotype (desensitization and rectification) to a different subset of the six possible subunit configurations (Figure 15).

1. Model 1 assumes that the inclusion of one or more marked subunits is sufficient to convert the phenotype of the assembled channel.

2. Model 2 assumes that the channel is converted to the marker phenotype when two or more marked subunits are present.

a. Model 2A assumes that the two marked subunits are present side by side (polar heterodimer).

b. Model 2B assumes that two marked subunits are located on opposite sides of the channel pore (specific point symmetry). 
3. Model 3 assumes that three or more marked subunits are required to convert the phenotype of the assembled channel.

4. Model 4 assumes that only symmetric assemblies are permitted configuration requires that two or more marked subunits convert the channel phenotype.

The model predictions depend on the channel conductances of the various subunit combinations. The phenotype expression models incorporate the average conductance wherever necessary for each AMPA channel configuration. We estimated the conductance parameters by performing single channel recording and mean-variance analysis experiments as described in section 3.8. Necessary for modeling required the consideration of relative conductance values for the various configurations.

\subsubsection{Construction of phenotype expression models based on single channel conductance}

We constructed a phenotype expression model based on the single channel conductance values for homomeric cotransfection between $\mathrm{R} 2(\mathrm{Q})$ and $\mathrm{R} 2(\mathrm{R})$. We assigned the estimated values of single channel conductance to different homo and heteromeric subunit combinations. The expected distribution of phenotypes from these models was plotted as a function of DNA fractions (Figure 15). These models were subsequently compared to the observed distribution to assign specific assemblies to specific phenotypes.

The normalized conductance was set both for homo and heteromers. For $\mathrm{R} 2(\mathrm{Q})$ homomers the normalized conductance was set to 1.7 . We set 0.8 as normalized conductance for heteromeric combination of $1 \mathrm{Q} / 2 \mathrm{R}(3: 1$ and $2: 2$ heteromers), and 1.3 for 1Q/2Q (3:1 and 2:2 heteromers, Figure 13-(b)-(d)). Previous experiments have shown that R2R homomers conduct less single channel current (Swanson et al., 1997b). Based on previous studies, the normalized conductance for R2R homomers was set to 0.05. For 1:3 heteromers in $1(\mathrm{Q}) / 2(\mathrm{R})$ (Figure 13-(e)) conductance was set to 0.05 , while 0.085 was set for $2 \mathrm{Q} / 2 \mathrm{R}$. For coexpression of $2 \mathrm{Q}+2 \mathrm{R}$ (marker), 2Q+2Q (marker), and 2R+2Q (marker) the relationship between the marker phenotype expression and fraction of marked subunits displayed different curve patterns as predicted by Model 1-3 (Figures 15A-15C).

To determine the accuracy of these parameters, we performed several 
cotransfection experiments using different subunit combinations. All these provided an excellent agreement between the predictions of binomial assembly models and homomeric coexpression data. As for example, the nonrectification data for $1 \mathrm{Q}+1 \mathrm{R}(\mathrm{L}-\mathrm{Y})$ and $2 \mathrm{Q}+2 \mathrm{R}(\mathrm{L}-\mathrm{Y})$ at $-30 \mathrm{mV}$ can be accurately predicted by Model $2 \mathrm{~A}$ while Model 2B describe the data well at $+30 \mathrm{mV}$ (see section 3.10.6.1, Figure 18A). One could not expect these internal consistent results if there was a serious error in the single channel current parameters for $\mathrm{Q} / \mathrm{R}$ assemblies. The error would have reflected to show a deviation of the phenotype expression from binomial model predictions.

A

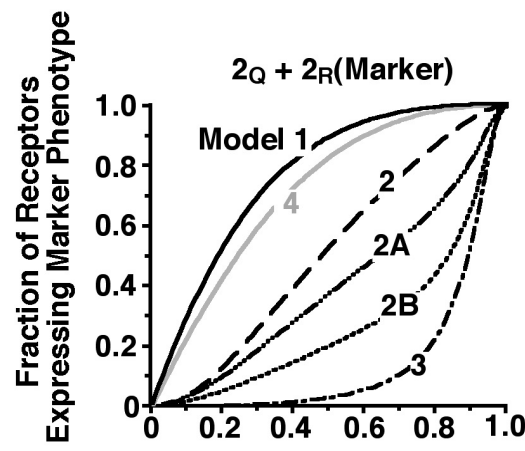

B

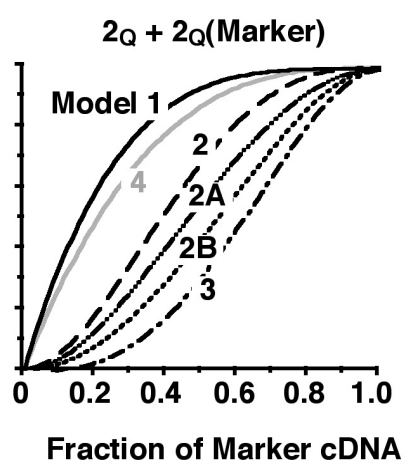

C

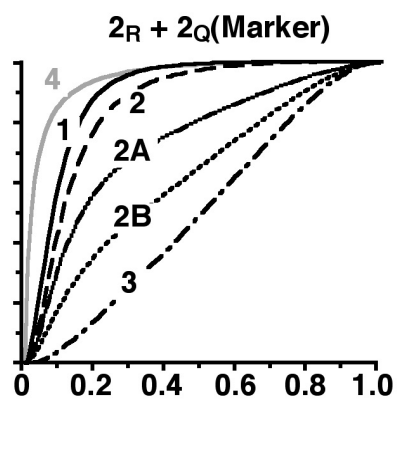

Figure 15. Phenotype expression pattern

(A) The figure represents the pattern of phenotype expression as predicted by the various models for cotransfection of wild-type R2 with marked R2 subunits. R2 subunit carries either R or Q residue in the channel pore region. We have plotted the fraction of receptors expressing the marker phenotype against the fraction of marked subunits. The pattern of curves generated for different models (Model 1,4,2,2A,2B and 3) are shown in the figure (B). The pattern of phenotype expression following cotransfection of $2 \mathrm{Q}$ with marked 2Q subunits are described by similar models. Note the difference between the shape of the curves when marker containing subunit is changed from arginine $(\mathrm{R})$ in the channel pore region to glutamine residue $(\mathrm{Q})$. (C) Pattern of phenotype expression following the cotransfection of $2 \mathrm{R}$ with marked $2 \mathrm{Q}$ subunits with similar models.

We confirmed the assumptions underlying the phenotype expression models by performing an extensive series of control experiments. We used markers for desensitization and rectification properties of individual subunit to address this question. In cotransfection experiments we show how the pattern of phenotype expression is different in homo and heteromeric assembly of AMPA receptors. 


\subsubsection{Desensitization and Rectification properties in Homo and Heteromeric assembly of AMPA receptors}

We performed a series of cotransfection experiments to know whether the pattern of heteromeric phenotype expression is different from homomeric combination using the markers for desensitization and rectification on either R1 or R2 subunit.

\subsubsection{Assembly of homomeric receptors}

To investigate the pattern of marker phenotype expression we tested different subunit combinations. The different combination tested for homomeric cotransfection experiments using markers for desensitization (L-Y) and rectification (Q-R) are represented above each figure. For the quantification of fraction of nondesensitizing receptors we utilized the combination of either R1Q or R2Q with nondesensitizing analogues $((\mathrm{R} 1(\mathrm{Q}) \mathrm{L}-\mathrm{Y}$ or $\mathrm{R} 2(\mathrm{Q})(\mathrm{L}-\mathrm{Y}))$. Rectification properties were quantified by combination of R2 subunit and its nondesensitizing analogue with R2(R) subunit.

\section{Desensitization}

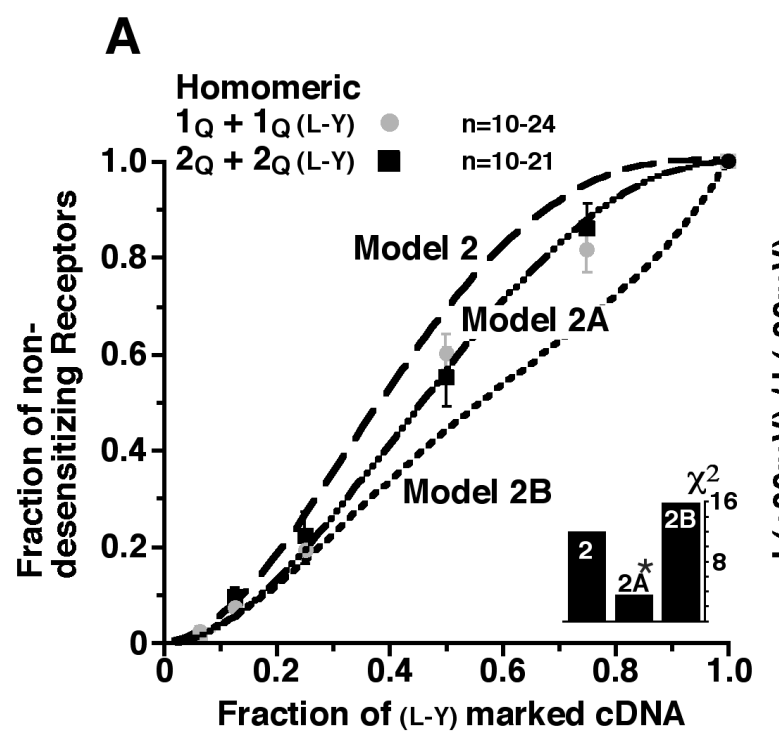

\section{Rectification}

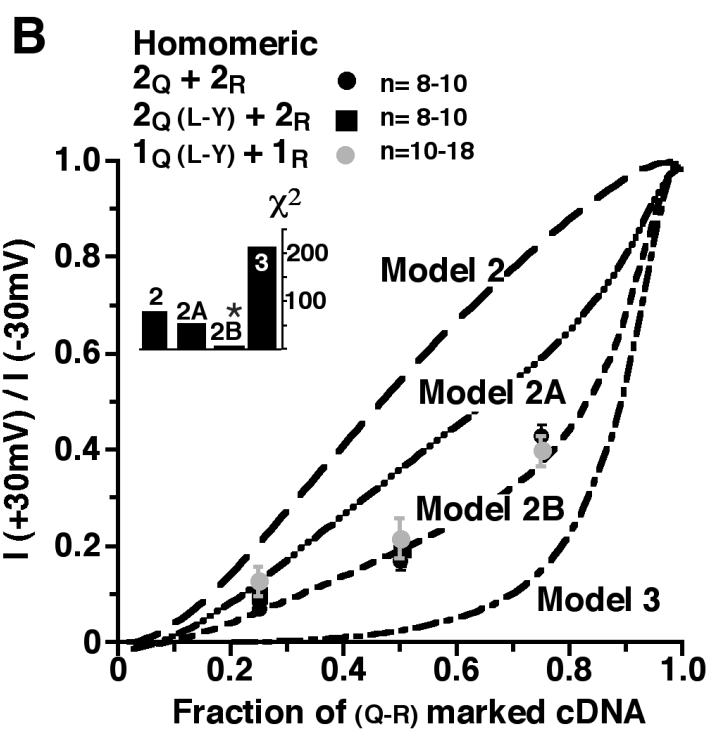

Figure 16. Assembly of homomeric subunit combinations in AMPA receptors

(A) Summary of results obtained from homomeric cotransfection experiments using markers for desensitization in $1 \mathrm{Q}+1 \mathrm{Q}(\mathrm{L}-\mathrm{Y})$ and $2 \mathrm{Q}+2 \mathrm{Q}(\mathrm{L}-\mathrm{Y})$ combinations. The plot represents the function of nondesensitization index against the fraction of DNA carrying the nondesensitizing L-Y marker. The data were compared to the predictions of Models 2, 2A, and 2B (dashed lines). Goodness of fit was assessed using the $\chi 2$ statistics (inset). (B) Summary of homomeric cotransfection experiments using markers for rectification in $2 \mathrm{Q}+2 \mathrm{R}$, (as shown by black circles) $2 \mathrm{Q}(\mathrm{L}-\mathrm{Y})+2 \mathrm{R}$ (as shown by black squares) and $1 \mathrm{Q}(\mathrm{L}-\mathrm{Y})+1 \mathrm{R}$ combinations (as shown by grey squares). 
The plot describes the function of nonrectification index against the fraction of cDNA carrying the nonrectifying Q-R marker, and compared to the predictions of Models 2, $2 \mathrm{~A}, 2 \mathrm{~B}$, and 3. The asterick mark in the inset corresponds to that model which represents the most appropriate theoretical fit for the data points. $\mathrm{n}$ represents the number of outside-out patches.

Furthermore, the pattern of expression for desensitization and rectification was tested for GluR1 and its nondesensitizing analogue with their non-rectifying analogue (GluR1(R)) (Figure 14B). The ratio of marked and unmarked subunit DNA was tested in a series of homomeric cotransfection experiments to quantify the amount of receptor penetrance and pattern of individual subunit expression in homomeric assembly of R1 and R2 receptors. The nondesensitization index (Figure 16A) and rectification index (Figure 16B) were plotted against the fraction of marked subunits in these combinations. We compared the data points with the predictions of the theoretical models (model 2,2A, 2B). The index for nondesensitization followed a clear pattern of Model 2A, while the index for nonrectification followed the behavior of Model 2B. We assessed the goodness of fit by using the $\chi 2$ statistics (inset).

\subsubsection{Assembly of heteromeric receptors}

The pattern of phenotype expression for heteromeric combination of subunits was tested employing the markers for desensitization and rectification on both GluR1 and GluR2 subunits. We observed that the pattern of expression in terms of desensitization and rectification for heteromeric cotransfections were clearly distinct from homomeric cotransfections.

The pattern of expression for the index of nondesensitization (Figure 17A) or nonrectification (Figure 17B) determined over different range of DNA concentration in heteromeric cotransfection experiments followed the behavior of theoretical superimposed Model 4. The inset shows the assesment of goodness of fit.

Thus two independent tests showed a large but consistent difference between assembly of homo and heteromers. Furthermore, the data suggest that the marker phenotypes for desensitization and rectification properties are expressed much more readily by heteromeric combination of receptors than by homomers. The internal consistency and reproducibility of the data strongly suggest that similar underlying 

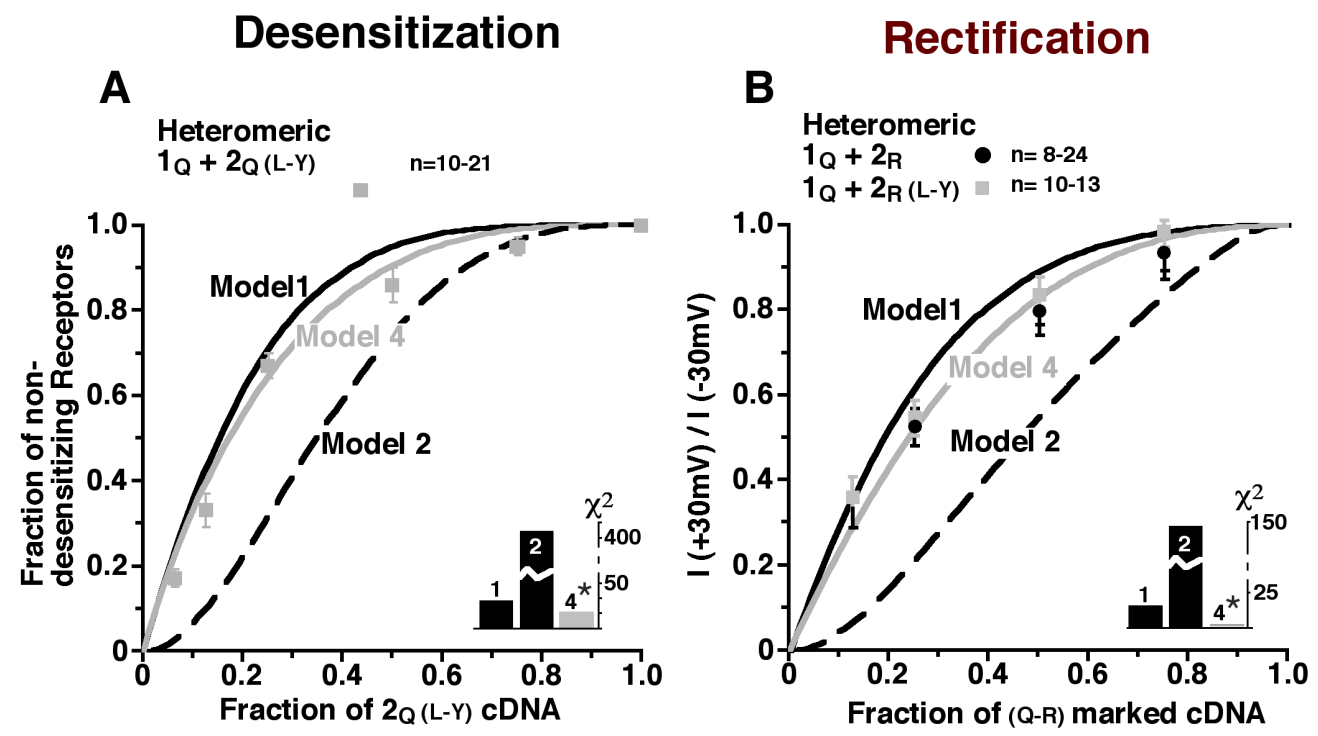

Figure 17. Assembly of heteromeric subunit combinations in AMPA receptors

(A) Summary of results obtained from heteromeric $1 \mathrm{Q}+2 \mathrm{Q}(\mathrm{L}-\mathrm{Y})$ cotransfection experiments using marker for desensitization. The results are compared to the predictions of Models 1, 2, and 4. Note that the data points corresponding nondesensitizing fraction of receptors are well fit by Model 4 as compared to other models (B) Summary of heteromeric cotransfection experiments using markers for rectification in $1 \mathrm{Q}+2 \mathrm{R}$ (marked by balck circles) and $1 \mathrm{Q}+2 \mathrm{R}(\mathrm{L}-\mathrm{Y})$ combination (marked by grey squares). These results are compared to the predictions of models 1 , 2 , and 4 . Note that the data points corresponding nonrectifying fraction of receptors are also well fit by Model 4 as compared to other models. Goodness of fit was assessed using the $\chi 2$ statistics (inset). $\mathrm{n}$ represents the number of outside-out patches.

mechanisms may be working in both the cases.

Based on the models as described in Figure 5 (section 3.5) one can predict that heteromeric subunits may not follow random pattern of assembly in contrast to homomeric subunits. This mechanism is counterintuitive because it implies that substituting an unmarked GluR2 subunit with an unmarked GluR1 subunit can change the marker phenotype. This requires that the substitution should affect both L-Y and Q-R phenotypes in a similar fashion. This explanation appears unlikely. Rather a simpler view that can explain heteromeric subunits assembly is their property of assembling preferentially in a particular configuration that allow them to express both the L-Y and Q-R phenotypes (Figure 5C, section 3.5). In lines with this hypothesis, the pattern of heteromeric phenotype expression is very similar for markers of desensitization and rectification (Figures 17A and 17B). We further confirmed our 
findings by cotransfecting wild-type subunits with the subunit carrying both the markers (desensitization and rectification).

\subsubsection{More evidences and consistent results in AMPA receptor assembly by altering the markers on different subunits}

We compared whether the desensitization and rectification properties of heteromeric receptor follows a differential behavior in comparison to homomeric receptors by changing both the markers (desensitization and rectification) between two subunits.

\subsubsection{Results on homomeric assembly of subunits}

Individual subunits (R1 or R2) were first made to carry both the markers (desensitization and rectification). An interchange of markers (L-Y and Q-R),

\section{Desensitization}

A

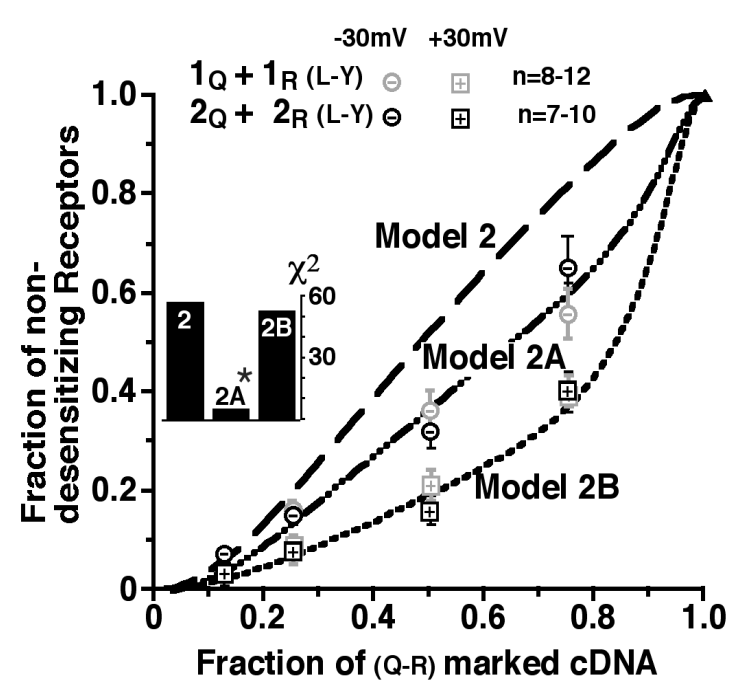

Rectification

B

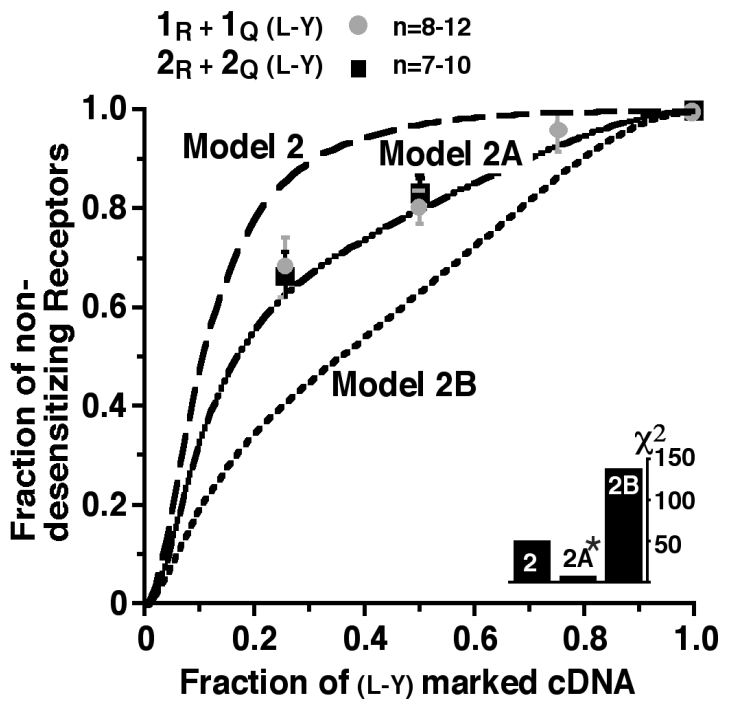

Figure 18. Additional evidences for homomeric AMPA receptor assembly by exchanging the markers for desensitization and rectification on different subunits

(A) Summary of the results obtained from homomeric $1 \mathrm{Q}+1 \mathrm{R}(\mathrm{L}-\mathrm{Y})$ and $2 \mathrm{Q}+2 \mathrm{R}(\mathrm{L}-$ $\mathrm{Y})$ cotransfection experiments. The plot describes nondesensitization index as a function of the fraction of cDNA carrying the L-Y marker. Experiments were carried out at $-30 \mathrm{mV}$ and $+30 \mathrm{mV}$. The fraction of nondesensitizing receptors at positive membrane potential was calculated as steady state current at $+30 \mathrm{mV} /$ steady state current at $-30 \mathrm{mV}$ in the presence of cyclothiazide. These data were compared to the predictions of Model 2, 2A, and 2B (dashed lines). The goodness of fit was assessed 
by $\chi^{2}$-statistics for the results obtained at $-30 \mathrm{mV}$ (inset). (B) Summary of results obtained from homomeric R1 and R2 subunits in $1 \mathrm{R}+1 \mathrm{Q}(\mathrm{L}-\mathrm{Y})$ and $2 \mathrm{R}+2 \mathrm{Q}(\mathrm{L}-\mathrm{Y})$ cotransfection experiments. We have plotted nondesensitization index against the fraction of cDNA carrying the L-Y marker. This is compared to the predictions of Models 2, 2A, and 2B.

resulted in phenotype expression data for homomeric receptors that was better described with a random assembly model (Figures 18A and 18B). The distribution of the amount of non-desensitizing receptors at $-30 \mathrm{mV}$ were described well with the predictions of Model 2A (based on the superimposition of the theoretical curve on the data points), while Model 2B was required to fit the data set for responses at $+30 \mathrm{mV}$. The rectification properties were described well with the predictions of Model 2A.

\subsubsection{Results on heteromeric assembly of subunits}

In heteromeric cotransfection experiments, the markers for both desensitization and rectification were changed between R1 and R2 subunits. Both the desensitization (Figure 19A) and rectification (Figure 19B) properties correlated well with the Model 4.

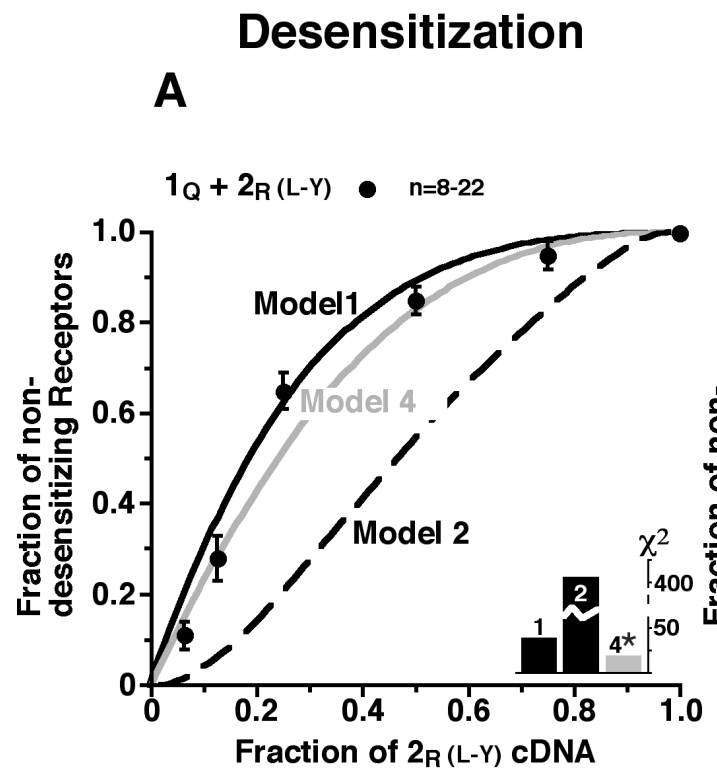

\section{Rectification}

B

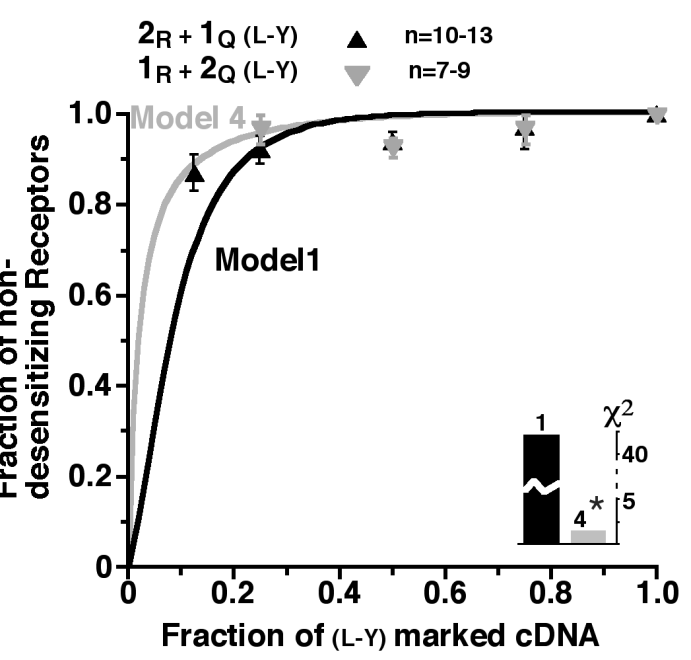

Figure 19. Additional evidences for heteromeric AMPA receptor assembly by exchanging the markers for desensitization and rectification on different subunits

(A) Summary of results obtained from heteromeric cotransfection experiments of $1 \mathrm{Q}$ $+2 \mathrm{R}(\mathrm{L}-\mathrm{Y})$ combination. The plot describes the function of nondesensitization index versus the fraction of $2 \mathrm{R}(\mathrm{L}-\mathrm{Y}) \mathrm{cDNA}$. The data is compared to the predictions of Model 1, 2, and 4. Note that Model 4 describes the data well than Model 1 and Model 
2 (B) Summary of results obtained from heteromeric cotransfection experiments in $2 \mathrm{R}$ $+1 \mathrm{Q}(\mathrm{L}-\mathrm{Y})$ and $1 \mathrm{R}+2 \mathrm{Q}(\mathrm{L}-\mathrm{Y})$ combinations. These results are compared to the predictions of Model 1 and 4. Hence the hypothesis of random assembly mechanisms for heteromeric receptors can be rejected (Figure 5B, Model 1).

Based on these observations, one can thus think of potential features that allow heteromeric subunits to assemble in a favorable manner. The selective specificity in turn should allow them to express the marker for desensitization (L-Y) and rectification (Q-R) phenotypes efficiently (Figure 5C, Model 4, section 3.5). To compile the selective specificity and phenotype expression properties within the assembly, the subunits should arrange with symmetry driven principles. Considering such principles, we have assumed arbitrarily in Model 4 that a symmetric configuration is favored when GluR1 and GluR2 subunits assemble as heteromers.

We next wanted to determine the actual subunit configuration of heteromeric AMPA receptors. To understand the subunit configuration it was essential to know the relation of spatial arrangement of marked subunits with respect to the channel phenotype. Based on the differential phenotypic expression of homo and heteromeric AMPA receptors, we show of how the channel properties in these receptors are controlled by the spatial arrangement of subunits.

\subsubsection{Channel properties of AMPA receptor are controlled by spatial arrangement of subunits}

\subsubsection{Control by desensitization}

The phenotype expression pattern clearly showed that homomeric subunits assemble in a random fashion. To examine whether the arrangement of the subunits affect channel gating, the markers for desensitization and rectification properties were exchanged between R1 and R2 subunit. Thus we cotransfected R2(Q) and R2(Q)(LY) receptors and examined the pattern of expression by plotting the nondesensitization index against marked cDNA fraction (Figure 16A). Identical results were observed when experiments were repeated for $1 \mathrm{Q}+1 \mathrm{Q}(\mathrm{L}-\mathrm{Y})$ combination (Figure 16A). Since all channels have the same conductance for these two subunit combinations, one can simplify the assumptions underlying the phenotype expression models. These results were best described by Model 2A (Figure 16A). The goodnessof-fit for the various models was assessed using the $\chi 2$ statistic (see insert). As an independent test, we examined the coexpressed $1 \mathrm{Q}+1 \mathrm{R}(\mathrm{L}-\mathrm{Y})$ and $2 \mathrm{Q}+2 \mathrm{R}(\mathrm{L}-\mathrm{Y})$ 
(Figure 18A). Although the predictions of the phenotype expression models were more widely separated due to relatively low conducting properties of $1 \mathrm{R}$ and $2 \mathrm{R}$ homomers, the data were accurately described by Model 2A (Figure 18A). Based on these results all the other models could be rejected $(\mathrm{p}<0.05)$. According to Model $2 \mathrm{~A}$ these result can be understood based on the spatial arrangement of subunits. The channels will be desensitizing if two marked subunits occupy opposite sides of the channel pore (symmetric heterodimer arrangement) and will have nondesensitizing character when marked subunits are positioned side by side (polar heterodimeric arrangement). These observations indicate that both the stoichiometry and spatial arrangement of the subunits influence the channel properties.

\subsubsection{Control by rectification}

We next addressed whether rectification properties of subunits followed a similar pattern of phenotype dominance in assembly. It is known that ion permeation through AMPA receptors is controlled by the presence of an arginine/glutamine in the pore-forming region of second transmembrane domain. The presence of edited version of GluR2 subunit (Q607R) in the assembled receptors can convert a rectifiying channel to linear one, while its absence results in their inward rectification due to intracellular spermine block (Dingledine et al., 1999). To verify these properties in assembly, we coexpressed the homomeric R2(Q) + R2(R) subunits and plotted the nonrectification index as a function of marked subunit fraction (Figure 16B). The goodness-of-fit for the various models was assessed using the $\chi^{2}$ statistics. Since the data were accurately described by Model 2B, all the other models could be rejected ( $\mathrm{p}<0.05$, Figure 16B insert). We observed the identical results even when their nondesensitizing analogs were present on either R1 or R2 subunits in cotransfection experiments. According to Model 2B one can understand the rectification properties based on spatial arrangement of subunits. The channels will have linear I-V relationship if two marked subunits occupy opposite sides of the channel pore (symmetric heterodimers) and will have rectifying character if they are positioned side by side (polar heterodimers). These observations strengthen the idea that both stoichiometry and spatial arrangement of the subunits influence the channel properties.

\subsubsection{Heterodimerization of AMPA receptors}


Our data suggested that both stoichiometry and spatial arrangement of subunits act as important parameters to control the channel properties. To crosscheck these results we performed cotransfection of wild-type homomeric subunit (R1(Q) and R2(Q)) with the subunit carrying both markers (R1R(L-Y),R2R(L-Y)) simultaneously. A typical raw trace for such cotransfection experiments at ratio 1:1 is shown in Figure 20.

\section{$1_{Q}+1_{R}(L-Y) \quad 3: 1$}

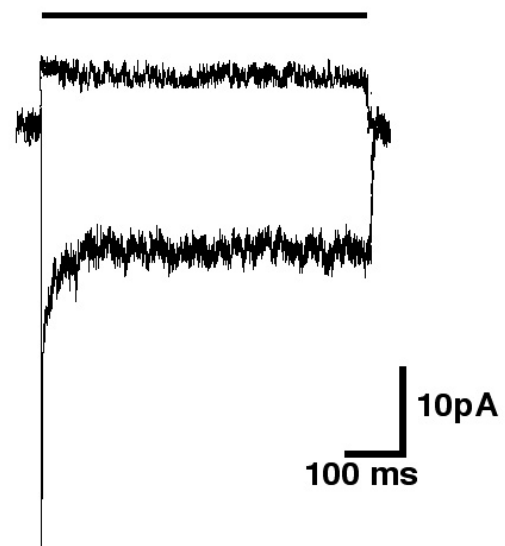

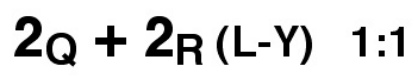

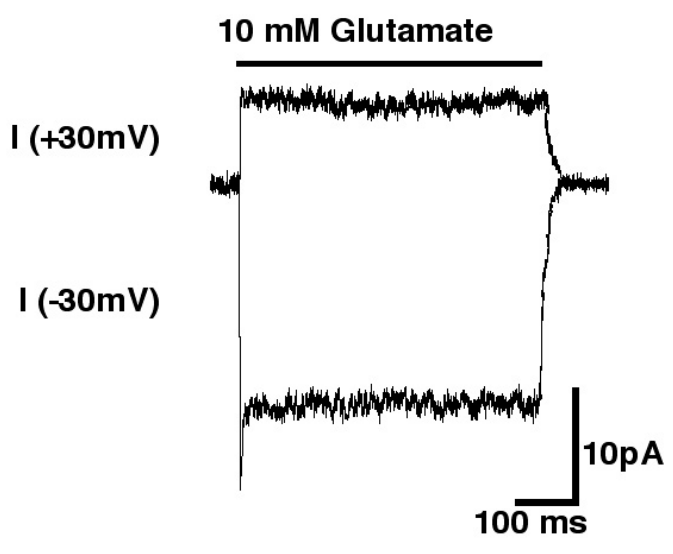

Figure 20. Raw traces from cotranfection experiments of unmarked with marked subunit for desensitization and rectification

A typical response obtained from a patch containing $1 \mathrm{Q}+1 \mathrm{R}(\mathrm{L}-\mathrm{Y})$ at a ratio of $3: 1$ (left) and $2 \mathrm{Q}+2 \mathrm{R}(\mathrm{L}-\mathrm{Y})$ (right) at a ratio of $1: 1$. The responses were recorded at negative $(-30 \mathrm{mV})$ and positive $(+30 \mathrm{mV})$ membrane potential. According to the predictions of Model 2A and 2B both responses should desensitize strongly at +30 $\mathrm{mV}$. However desensitization was not observed in these responses.

We plotted the desensitization indices as a function of marked subunit fraction at both $-30 \mathrm{mV}$ and at $+30 \mathrm{mV}$ (Figure 18A). Based on these above results, symmetric heterodimers should desensitize as the desensitization index follows the prediction of Model 2A. In terms of rectification, these results imply that symmetric heterodimers should be nonrectifying since nonrectification index follow the prediction of Model 2B. However, we observed that the steady state response at $+30 \mathrm{mV}$ was large (about $50 \%$ of the response at $-30 \mathrm{mV}$ ) and did not exhibit any desensitization (Figure 20). 
This was contradictory to our expectation that the response at $+30 \mathrm{mV}$ should desensitize strongly resulting in a very small steady-state current. To explain these results we had to discard Model 2B. A new phenotype expression model was constructed based on refined assumptions. We assumed that (a) in tetrameric AMPA receptors, the subunits are structured as pair of dimers (b) nondesensitizing receptors must have at least one L-Y marker in both dimers. This is shown in the following figure by Model 2C (Figure 21A).

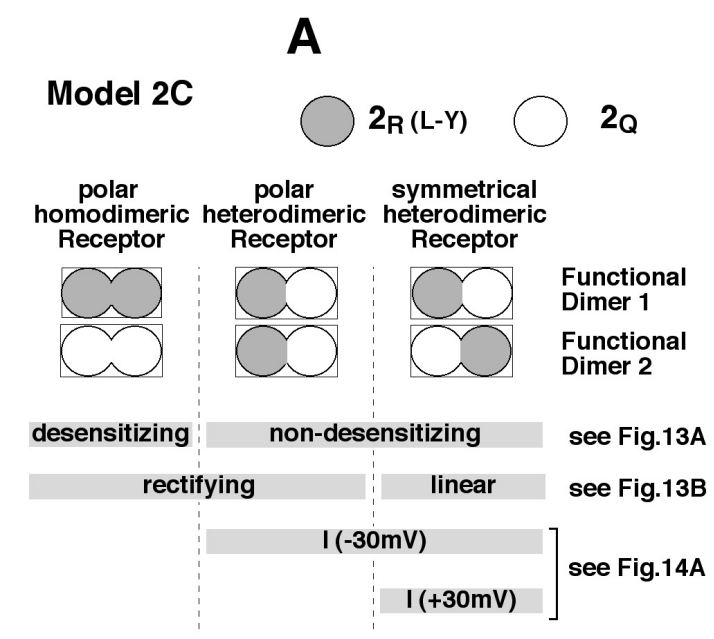

B

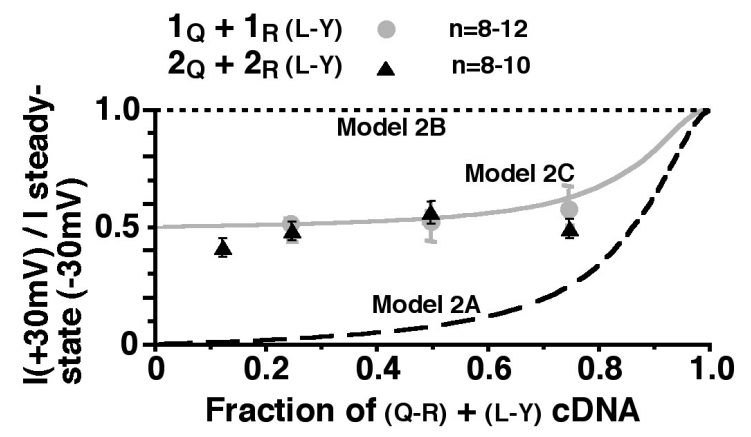

Figure 21. Distribution of marker phenotypes in different subunit arrangements in heterodimer

(A) Model 2C predicts the distribution of marker phenotypes for 2:2 heteromeric combinations. This is based on the assumption that AMPA receptors assemble and function as a pair of dimers. The classification of desensitizing and rectifying properties of 2:2 heterodimers are shown for receptors assembled from 2Q (white circles) and $2 \mathrm{R}(\mathrm{L}-\mathrm{Y})$ (gray circles). The pairs of fused circles represent functional dimers within the assembled receptor. Classification of heterodimer into polar and symmetric form is shown in this cartoon (B) A plot representing the ratio of 
nondesensitizing outward current and the steady-state inward current for $1 \mathrm{Q}+1 \mathrm{R}(\mathrm{L}-\mathrm{Y})$ and $2 \mathrm{Q}+2 \mathrm{R}(\mathrm{L}-\mathrm{Y})$ in cotransfection experiment. The phenotype expression patterns predicted by different Models (2A, 2B, and 2C) are superimposed.

Model 2C is based on the idea that desensitization of receptor is blocked when the tyrosine residue is present at the dimer interface in both pair of dimers. Model 2C predicts that polar homodimeric receptor should desensitize and rectify while symmetric heterodimers will not desensitize and have nonrectifying (linear) behavior. Based on the predictions of model $2 \mathrm{C}$, the steady-state current at positive membrane potentials $(+30 \mathrm{mV})$ should be approximately half the value of steady-state current at negative potentials $(-30 \mathrm{mV})$. We observed that the data were consistent with the predictions of model (Figure 21B).

We plotted the ratio of steady-state current at positive and negative potentials against the fraction of L-Y mutant cDNA concentration. We compared these results with the model predictions (Figure 21B). We observed that the results strongly favor Model 2C as compared to Model 2A or 2B. This supports the hypothesis that AMPA receptor subunits are assembled as pair of dimers (Armstrong and Gouaux, 2000). Our results are consistent with recent structural elucidation of dimerization observed in GluR2 receptors. In the crystal structure (assuming the tetrameric arrangement of subunits and the dimeric nature of ligand-binding core) the two dimers are placed side by side in tetrameric arrangement. The interdimer 2 -fold is in the center of the 4 subunits and coincides with the channel 4-fold.

A cross verification was made in a cotransfection of R2(Q) with $2 \mathrm{R}(\mathrm{L}-\mathrm{Y})$ at 1:1 cDNA ratio. We observed a similar result of nondesensitizing outward current at $+30 \mathrm{mV}$ similar to GluR1 subunit combination. Outward current was not potentiated by $100 \mu \mathrm{M}$ cyclothiazide $(0.98 \pm 0.02, \mathrm{n}=12)$. These data are consistent with Model $2 \mathrm{C}$ and not in accordance with Model 2A. If one expects 1:3 heteromers and $2 \mathrm{R}$ homomers (nonrectifying and desensitizing in cotransfection experiments) then, according to Model 2C, they should have very low conductance. These results also give additional information in support to the conductance parameters that are extensively used in the phenotype expression models. Furthermore, we observed that the steady-state amplitude of the response at $-30 \mathrm{mV}$ for $2 \mathrm{Q}+2(\mathrm{R}) \mathrm{L}-\mathrm{Y}$ was weakly potentiated by cyclothiazide $(13 \% \pm 3 \%, \mathrm{n}=12)$. According to the predictions of Model 2C the potentiation of cyclothiazide should be approximately $20 \%$ which is close to the observed potentiation. These results are consistent with the idea that polar 
homodimeric receptors would desensitize and hence are potentiated by cyclothiazide (Figure 18A).

These data suggest that within a tetrameric AMPA receptor subunits are structured as a pair of dimers. Secondly, both the stoichiometry and the spatial organization of marked subunits (desensitization and rectification) within a channel determine its phenotype. These collective informations served to determine the preferred subunit configuration of heteromeric AMPA receptors.

\subsubsection{Subunit configuration for heterodimeric assembly of $A M P A$ receptors}

We already discussed of how desensitization and rectification properties of subunits control the channel phenotype within AMPA heteromers. We found that R1 and R2 subunits should follow a specific geometric configuration to assemble in a heterodimeric fashion. A marker for either nondesensitization or nonrectification (linearization) on R2 subunit allowed the preferred configuration of subunits to express the marker phenotype effectively (Figures $13 \mathrm{c}$ and $13 \mathrm{~d}$ section, 3.10.1). If one assumes that the same rules of phenotype expression are followed by homomeric and heteromeric receptors during assembly, then one can determine the preferred subunit configuration that allows them to assemble. The phenotype expression determined by nondesensitization marker does not require additional support of evidences from single channel conductance. However the interpretation for phenotype expression determined by nonrectifying marker relies on the conducting properties of subunit configurations and their contribution for phenotype expression. Furthermore, the assumptions of model $2 \mathrm{C}$ and the observed result rules out the possibility of contribution of 1:3 heteromers in determining the phenotype dominance. Eliminating these possibilities heteromeric AMPA receptors must preferentially assemble in symmetric heterodimer configuration.

\subsubsection{Possible mechanisms for subunit assembly of AMPA receptors}

\subsubsection{Symmetry controlled mechanism}

The phenotype expression model provides no insight into the molecular mechanisms that might be responsible for selective assembly of subunits. One can 
consider simple geometric factors such as different intersubunit bond lengths for R1 and R2 subunits that might be responsible for favoring a symmetric heterodimeric configuration. Symmetric arrangements involve the right and left hand interface matching of one subunit with the other. The misalignment of subunit-subunit interfaces or steric hindrance may destabilize the asymmetric subunit configurations. This is schematically represented in the following cartoon (Figure 22). These cartoons present a simple, physically plausible picture of potential mechanisms underlying selective assembly. We further show of how the model predictions fit the experimental data set.

A Symmetry Controlled Assembly Model 4

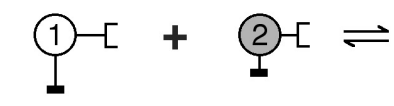

Symmetric Assemblies

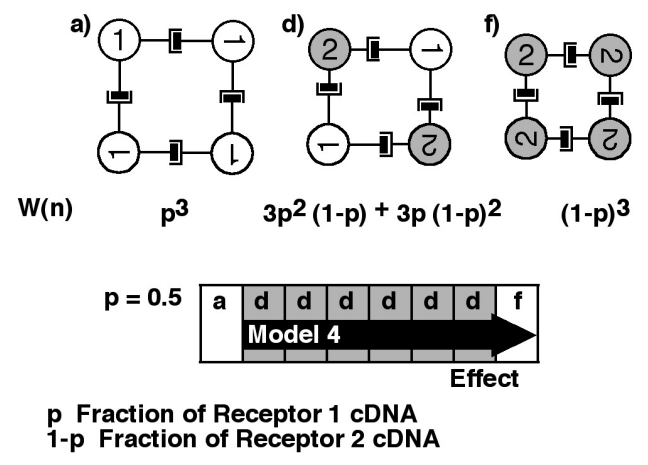

B Asymmetric Assemblies
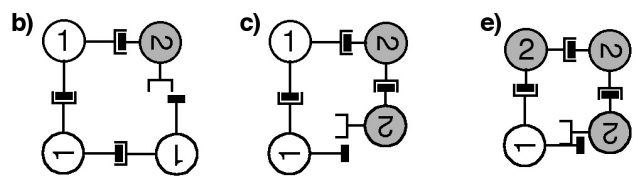

Figure 22. Schematic representation of symmetric and asymmetric configurations during AMPA receptor assembly.

(A) The cartoon describes the possible configurations in symmetry controlled assembly between subunits 1 and 2 (for GluR1 and GluR2 subunits) when they interact with each other. Symmetric arrangement allows either preferred configuration for heteromeric coassembly (d) or permitted configuration for homomeric subunit combinations $(\mathrm{a}, \mathrm{f})$. In symmetry controlled subunit assembly if one assumes that subunit-subunit binding is reversible then the expressed subunits will be present in one of the three stable symmetric configurations. This would then lead to binomial distribution as described in the Model 4 (B) Asymmetric configuration (b,c,e) may arise from misalignment of subunit-subunit interfaces or steric factors due to 
difference in the bond length.

\subsubsection{Association controlled mechanism}

The second hypothesis regarding subunit assembly mechanism assumes that the consecutive addition of monomers lead to formation of a dimer. The dimer of a dimer will finally form a full tetramer. One proposed model of the controlled assembly is described in figure 23. Based on these pictorial descriptions one can theoretically model the data set in cotransfection experiments to extract the rate of association between R1 and R2 subunits. Shown in the figure 23B are few theoretical models imposed on the actual data set. The heteromeric subunits 1 and 2 are assigned with a different rate of association $\left(\mathrm{k}_{21}\right)$ as compared to homomeric combination $\left(\mathrm{K}_{11}\right)$. This is due to differential surface interaction between subunits in homo and heteromers. Furthermore, the reaction rates will differ based on right-hand and left hand interactions. As for example, if the right-hand face of subunit 1 binds to the lefthand face of subunit 2 the reaction rate will be $k_{12}$ while, if the left-hand face of subunit 1 binds to right-hand face of subunit 2 the reaction rate will be $k_{21}$. The best fit was obtained when the rate of association between R1 and R2 subunit was assumed to be five times faster. This assumption is along with the idea that dimers are favored with fast association rate as compared to other forms such as monomers. Furthermore, it is consistent with R2 subunit which dimerizes with R1 subunit more rapidly than other subunit pairings. These mechanisms may be favorable if the physical profile of the right-hand interface of R2 subunit matches closely with the

A Association Controlled Assembly Model 5
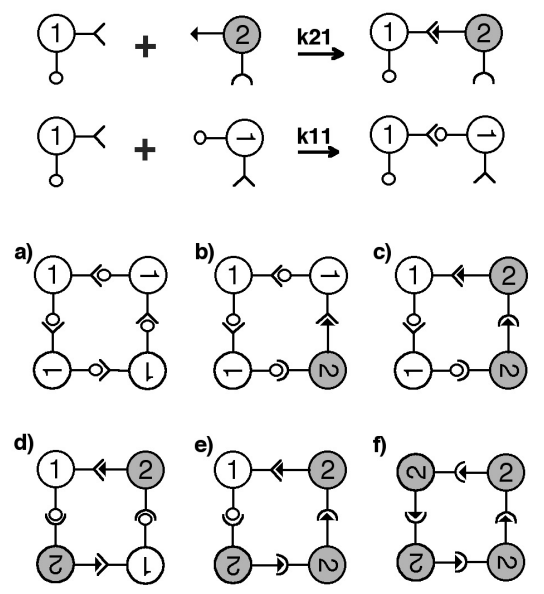

B

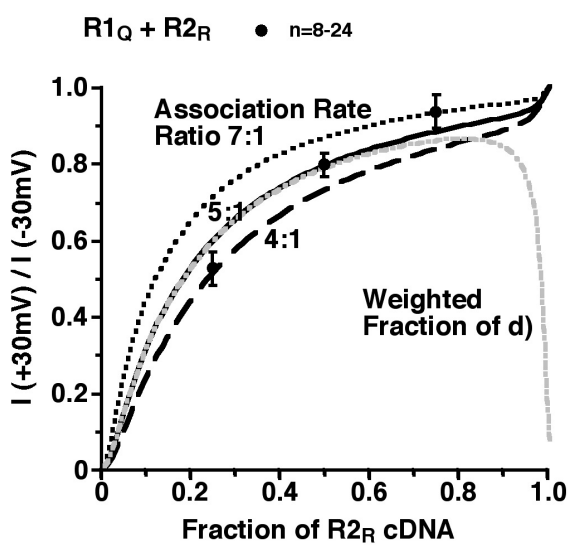

Figure 23. A cartoon representing the association controlled assembly process 
(A) 1 and 2 corresponds to $\mathrm{R} 1$ and $\mathrm{R} 2$ subunits. $\mathrm{k}_{11}$ and $\mathrm{k}_{21}$ represents the association rate of binding between homo and heteromers formed between R1 and R2 subunits. $\mathrm{a}, \mathrm{b}, \mathrm{c}, \mathrm{d}, \mathrm{e}$ corresponds to the locking of subunits either in homo or heteromeric fashion. $\mathrm{a}, \mathrm{f}$ corresponds to the formation of pure homomers, while b,c,d,e corresponds to formation of heteromers. $\mathrm{c}$ and $\mathrm{d}$ corresponds to dimer formation where the identical subunits are either adjacent or are opposite to each other. (B) Theoretical models imposed on data set for cotransfection of $\mathrm{R} 1(\mathrm{Q})$ and $\mathrm{R} 2(\mathrm{R})$ subunit at 1:1 ratio. The different curves represent the fits corresponding to different association rates. $7: 1,5: 1,4: 1$ corresponds to the association rate of $\mathrm{R} 1$ with $\mathrm{R} 2$ subunit at 7,5 and 4 times faster than other subunit combinations.

left-hand interface of R1 subunit (association-controlled assembly).

Further experiments are necessary to understand of how the recognition sites are induced between pair of dimers to form a full tetrameric AMPA receptor. Such a paradigm would allow the subunits themselves to guide subunit interactions and to determine the assembly pathway by which they oligomerize.

From a number of experiments with varying subunit combinations using markers for either desensitization or rectification, we observed that the only two configurations that express both marker phenotypes are symmetric heterodimers. In summary, we have described the mechanistic principles for the preferential heteromeric AMPA receptor assembly. Furthermore, R1 and R2 assemble with a preferred subunit configuration in symmetric heterodimeric fashion.

\subsection{Effect of modulators on $A M P A$ receptors}

With the basic understanding of how desensitization and rectification properties of GluR1 and GluR2 control the assembly of homo and heteromeric receptors, we now study the effect of AMPA modulators that affect the gating properties in these receptors.

\subsubsection{Effect of modulators on recombinant AMPA receptors}

In this study we describe the modulator action of structurally different AMPA modulators on AMPA receptors. This is shown in Figure 1. 
<smiles>NS(=O)(=O)c1cc2c(cc1Cl)NC(C1CC3C=CC1C3)NS2(=O)=O</smiles>

Cyclothiazide<smiles>O=C(c1ccc2c(c1)NC=CN2)N1CCCCC1</smiles>

CX516<smiles>CC1Nc2ccc(Cl)cc2S(=O)(=O)N1</smiles>

IDRA 21<smiles>O=C(c1ccc2c(c1)OCCO2)N1CCCCC1</smiles>

CX546

Figure 1. The chemical structure of AMPA receptor desensitization modulators

Cyclothiazide and IDRA 21 belong to class of benzothiadiazine derivatives. CX516 and CX546 belong to benzoylpiperidine derivatives.

\subsubsection{Effect of CX546 on recombinant GluR1 and GluR2 subunits}

To determine the effect of CX546 on AMPA receptor subunits, we used flip splice variants of wild-type unedited R1 and R2Q receptor. We transiently transfected these subunits in HEK293 cells (Chen and Okayama, 1988). Outside-out patches were excised from these cells and were exposed to fast flow application system containing control and agonist solution. We performed these experiments both in the absence and presence of the modulator (Clements and Westbrook, 1991). A representative current trace recorded from wild-type R1(Q) and R2(Q) subunits expressed in HEK 293 cells is shown Figure 2 A, B, C. 
A

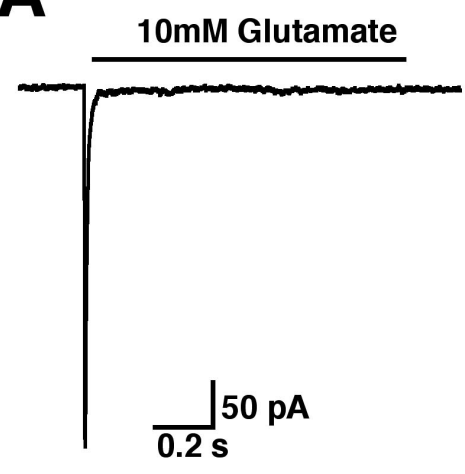

GluR1Flip

C

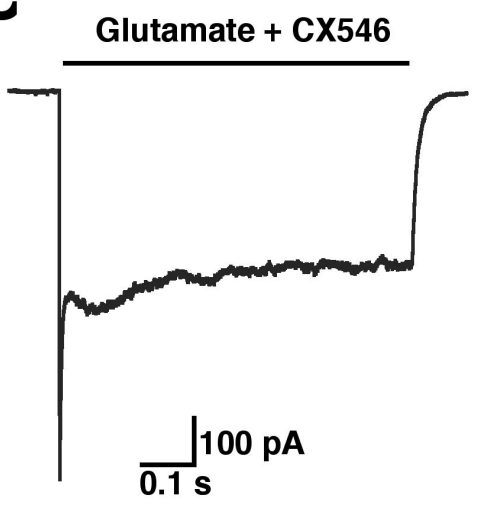

GluR2 Flip
B

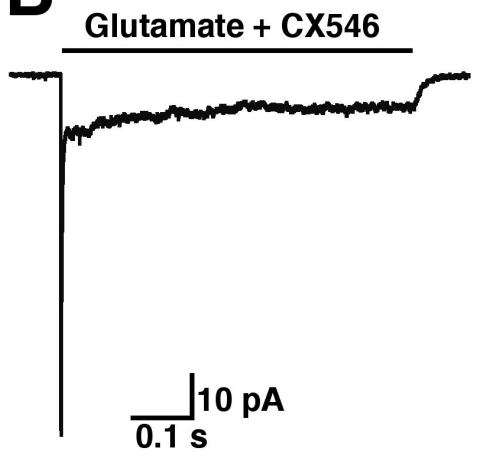

GluR1 Flip

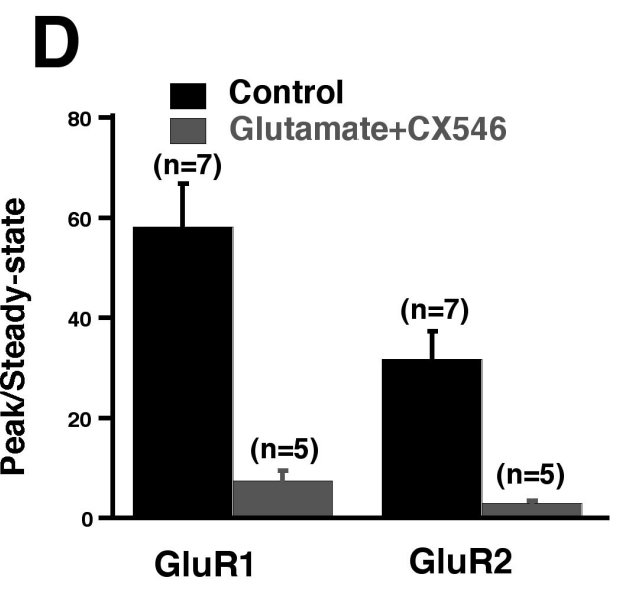

Figure 2. Effect of CX546 on recombinant GluR1 and GluR2 receptors

(A) Current response evoked by application of saturating concentration (10$\mathrm{mM}$ ) glutamate from an outside-out patch excised from transiently transfected HEK cells expressing $\mathrm{R} 1_{\text {flip }}$ subunit. $(\mathrm{B}, \mathrm{C})$ Representative traces of $\mathrm{R} 1_{\text {flip }}(\mathrm{B})$ and $\mathrm{R} 2_{\text {flip }}$ receptors $(\mathrm{C})$ in the presence of $300 \mu \mathrm{M}$ CX546 coapplied with glutamate $(10 \mathrm{mM})$. (D) Analyses of the amount of desensitization by peak to steady state current measurements $(\mathrm{P} / \mathrm{S})$. Holding potential was $-60 \mathrm{mV}$; Removal of desensitization was more complete in $\mathrm{R} 2$ receptors although the relative decrease in peak to steady state values were similar in $\mathrm{R} 1$ and $\mathrm{R} 2$ subunits. $\mathrm{n}$ represents the number of outside-out patches used for analysis. The values correspond to mean \pm SEM.

The currents were evoked in saturating concentration of glutamate $(10 \mathrm{mM})$. Intrinsic gating of the channel under these conditions can be studied without the channel being affected by binding and unbinding of the agonist. A long pulse (1 s) of glutamate application for R1 receptors (Figure 2A) evoked a rapidly desensitizing response which desensitized to less than $5 \%$ of the peak current. The degree of desensitization (Peak/Steady state current, represented as P/S hereafter) and the time course of desensitization ( $\tau$ des) were used as parameters to characteristize the properties of desensitization. We observed that the time course of desensitization 
( $\tau$ des) for R1 and R2 receptors was $3.5 \pm 0.3 \mathrm{~ms}(\mathrm{n}=15)$ and $8.8 \pm 0.6(\mathrm{n}=20)$ respectively. The P/S ratio was $66 \pm 6(n=7)$ for GluR1 subunit and $25 \pm 3(n=10)$ for R2 subunit respectively. When CX546 $(300 \mu \mathrm{M})$ was coapplied with glutamate (10 $\mathrm{mM}$ ) we noticed a partial removal of desensitization. This was apparent by the change in $\mathrm{P} / \mathrm{S}$ ratio for both $\mathrm{R} 1$ and $\mathrm{R} 2$ receptors. Though we observed a similar relative change in $\mathrm{P} / \mathrm{S}$ (9-fold reduction) for both R1 and R2 subunit, treatment of R2 subunit with CX546 resulted in a much higher steady-state enhancement of the current amplitudes than for GluR1. This is because R1 receptors desensitize more completely than $\mathrm{R} 2$ in the absence of CX546 (figure 2B,C). The P/S ratio was $7.3 \pm 1.9(13.7 \%$ of peak current, $n=5)$ for $\mathrm{R} 1$ and $2.7 \pm 0.4(37 \%$ of peak current, figure $2 \mathrm{C}, \mathrm{n}=5)$ for the R2 subunit in the presence of CX546. Surprisingly, we did not observe a significant change in the time courses of desensitization for $\operatorname{R} 1\left(\tau_{\mathrm{des}}=2.7 \pm 0.1 \mathrm{~ms}(\mathrm{n}=4)\right)$ and $\mathrm{R} 2$ $\left(\tau_{\mathrm{des}}=6.7 \pm 0.7 \mathrm{~ms}(\mathrm{n}=7)\right)$ subunits in the presence of CX546 despite the increased steady-state current. This implies that CX546 does not act by slowing the rate of receptor entry into the desensitized state (as is known from benzothiadiazide group of modulators), rather it seems to destabilize the desensitized state by increasing the rate out of the desensitized state.

\subsubsection{Effect of CX516 and IDRA-21 on recombinant GluR1 and GluR2 receptors}

We compared the modulatory action of CX546 with a structural analogue CX516. As compared to untreated patches, we did not observed any significant effect when patches from R1 receptors were exposed to CX516 at a concentration of 300$\mu \mathrm{M}$. The responses desensitized with a time constant $3.9 \pm 0.3 \mathrm{~ms}(\mathrm{n}=4)$ and the $\mathrm{P} / \mathrm{S}$ ratio was $45 \pm 12(n=3$, data not shown) in treated patches. The time constant of desensitization for the $\mathrm{R} 2$ receptor was slightly slowed to $13.5 \pm 1.6 \mathrm{~ms}$ which was not significant (Figure 3A left panel, insert, $n=3$ ). However the steady-state current increased significantly $(\mathrm{P} / \mathrm{S}, 11.6 \pm 0.8, \mathrm{n}=3)$ suggesting that $\mathrm{CX} 516$ may act in a similar fashion to CX546 but with lower efficacy at this concentration. These results are consistent with the previous observations in PFC pyramidal neurons (Baumbarger et al., 2001). 
A

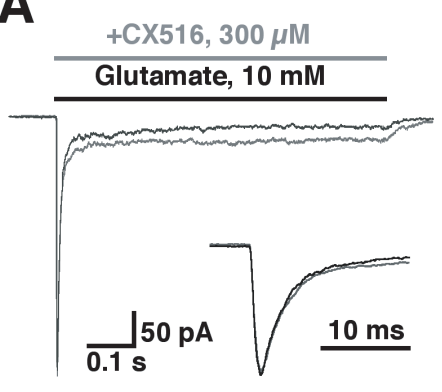

B

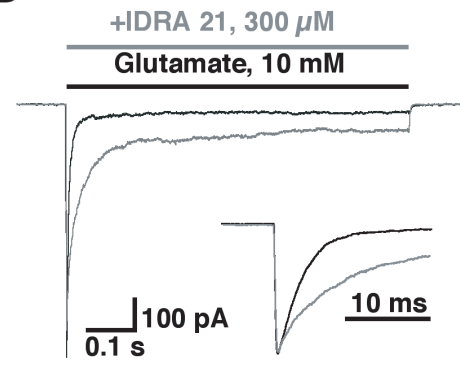

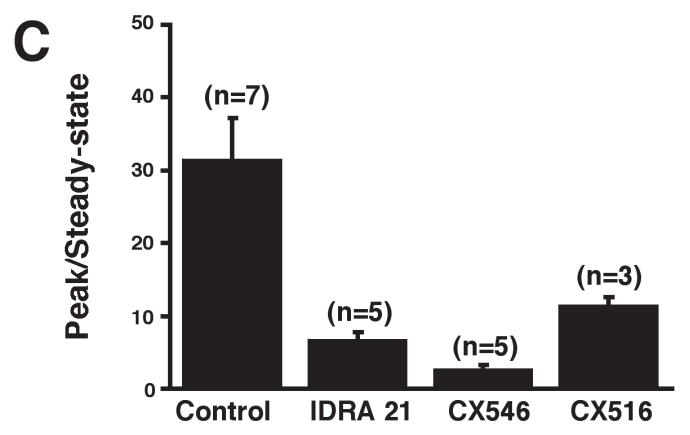

Figure 3. Modulation of desensitization by IDRA 21 and CX516

(A) Typical current responses of R2Q to glutamate $(10 \mathrm{mM})$ in the absence (black traces) and in the presence (gray traces) of $300 \mu \mathrm{M}$ CX516 (A) and $300 \mu \mathrm{M}$ IDRA 21 (B). Inserts show the onset of response on an expanded time scale. IDRA 21 slowed the time constants of desensitization. (C) Peak/steady-state ratios of untreated (control) and patches treated with the modulators.

IDRA21 has been shown to reduce AMPA receptor desensitization and slow the rate of deactivation in contrast to the modulatory action of ampakines (Yamada, 1998). The time course of desensitization in the presence of IDRA21 was best fit with two time constants $\left(\tau_{\text {fast }}=11.3 \pm 2.4 \mathrm{~ms} / \mathrm{Ampl}_{\text {fast }} 30 \pm 5 \%\right), \tau_{\text {slow }}=70 \pm 12 \mathrm{~ms} / \mathrm{Ampl}_{\text {slow }}$ $51 \pm 3 \%, n=6$,Fig $3 \mathrm{~B}$ right panel). Figure $3 \mathrm{C}$ summarizes the effect of different modulators on the amount of desensitization $(\mathrm{P} / \mathrm{S}$ ratio).

\subsubsection{Effect of modulators in the absence of desensitization}

\subsubsection{Effect of CX516 and IDRA 21 on non-desensitizing GluR1 receptors}

To understand if there are any side effects caused by these allosteric modulators besides desensitization, we tested them on the nondesensitizing mutant variant of R1 subunit (R1L497Y) (Stern-Bach et al., 1998). If there are any effects that are unrelated to desensitization such as agonist binding or fast channel block, 
coapplication of modulator should affect these responses. If the responses are not affected by coapplication of the modulators then one can conclude that other side effects of these modulators beside modulating receptor desensitization are unlikely to occur. As for example, cyclothiazide, a potent blocker of AMPA receptor desensitization leads to a $10-15 \%$ reduction of single channel currents (Partin et al., 1993; Rosenmund et al., 1998). Besides, it also reduces the apparent agonist affinity by a factor of 2-3 (Kessler et al., 1996; Stern-Bach et al., 1998).

A long application (1s) of saturating concentration of glutamate to outside-out patch of R1(L497Y) expressing cells led to a completely nondesensitizing response as expected. We did not observe a change in the response amplitude or shape $(n=4-7$, data not shown) by the application of the modulators CX516 and IDRA 21 at 300 $\mu \mathrm{M}$ concentration. These results suggest that CX516 and IDRA 21 at $300 \mu \mathrm{M}$ does not affect the gating properties for nondesensitizing AMPA receptors, desensitization aside, and does not cause the channel block. The shift in the apparent agonist affinity was tested by comparing the responses evoked by saturating agonist concentration (10 $\mathrm{mM}$ glutamate) with the response evoked by approximately halfmaximal agonist concentrations (10 $\mu \mathrm{M}$ glutamate). We observed that the relative response ratios in the absence and presence of modulators remain unchanged (control: $0.55 \pm 0.03$, $\mathrm{n}=21$; IDRA21: $0.53 \pm 0.04, \mathrm{n}=12 ;$ CX516:0.52 $\pm 0.03, \mathrm{n}=7$, Figure 4). Data shown here corresponds to the effect of CX516 alone. Both the modulators IDRA 21 and CX516 did not affect the agonist binding kinetics at this concentration $(300 \mu \mathrm{M})$.

\section{Glutamate}

\section{$10 \mathrm{mM}$}

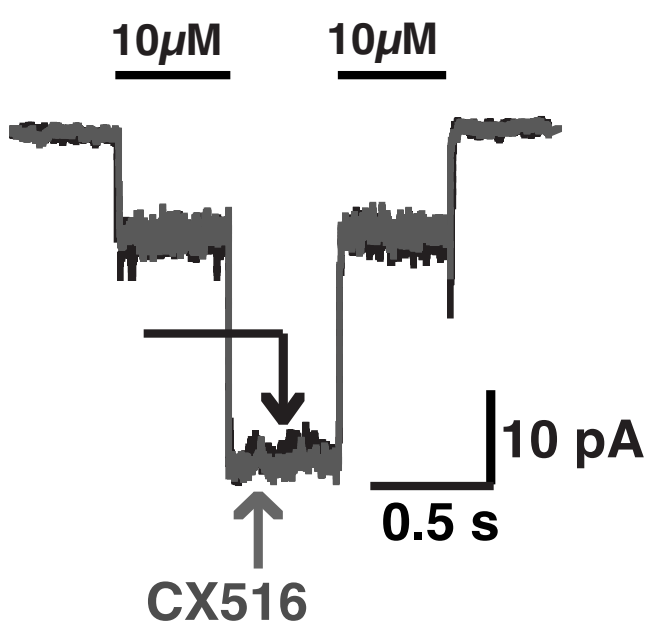


Figure 4. Absence of the effect of CX516 on nondesensitizing GluR1 L497Y receptor

Outside out patch responses excised from HEK cells expressing GluR1L497Y receptors were exposed to application (black bars) of half maximal $(10 \mu \mathrm{M})$ and saturating $(10 \mathrm{mM})$ concentration of glutamate. The responses were unaffected by coapplication of CX516 $(300 \mu \mathrm{M})$ suggesting that CX516 does not affect the affinity of agonist for receptor The control trace (black) and modulator trace (gray) are overlayed. These recordings were done by Christoph Quest.

\subsubsection{Effect of CX546 on nondesensitizing GluR1 receptors}

When outside out patches excised from R1L497Y were exposed to CX546 $(300 \mu \mathrm{M})$ in the presence of glutamate at submaximal $(10 \mu \mathrm{M})$ and saturating $(10 \mathrm{mM})$ concentration of glutamate, we observed an increase in the response amplitude at 10$\mu \mathrm{M}$ of glutamate, but not at $10 \mathrm{mM}$ concentration ( $\mathrm{n}=5$, Figure 5, left panel). This result suggests that, the affinity of glutamate for $\mathrm{R} 1$ mutant receptor is enhanced in the presence of CX546.

$\underline{\mathrm{CX546}} \quad \underline{\mathrm{CX546}}$

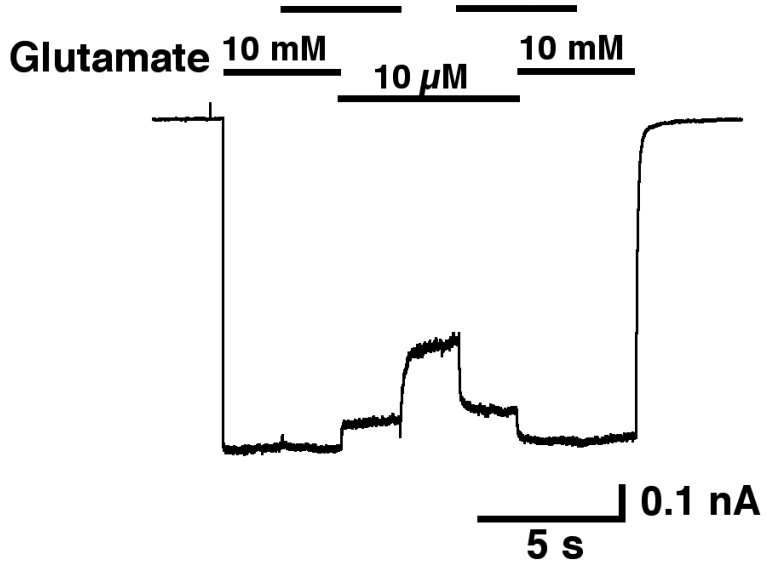

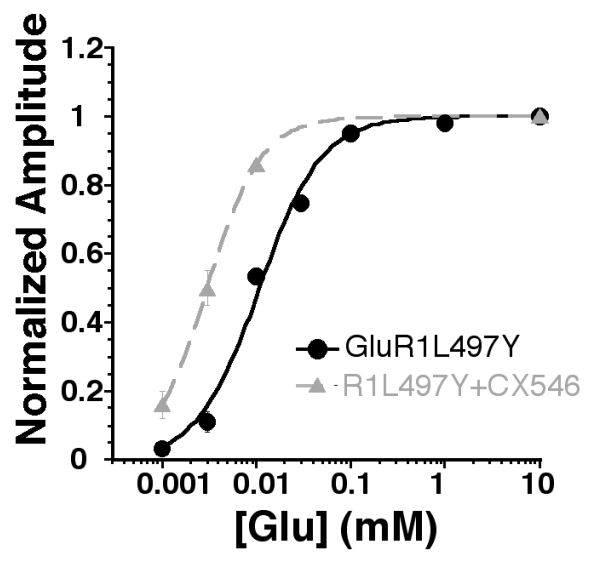

Figure 5. Apparent change in the affinity of glutamate for GluR1 L497Y in the presence of $C X 546$

Outside out patch responses excised from HEK cells expressing R1L497Y receptors were exposed to application (black bars) of half maximal $(10 \mu \mathrm{M})$ and saturating $(10-$ $\mathrm{mM})$ concentration of glutamate. The responses to the coapplication of CX546 $(300 \mu \mathrm{M})$ were affected at $10 \mu \mathrm{M}$ glutamate but remain unaffected at saturating concentration $(10 \mathrm{mM})$ glutamate (left panel). Dose response curve of glutamate on $\mathrm{R} 1 \mathrm{~L} 497 \mathrm{Y}$ in the absence (black dots, hill fit as black curve, $\mathrm{EC}_{50} 10.7 \mu \mathrm{M}$ ) and in presence of $300 \mu \mathrm{M}$ CX546 (gray triangles, hill fit as gray curve, $\mathrm{EC}_{50}=3.0 \mu \mathrm{M}$ ) (right panel). Data represents mean $\pm \mathrm{SE}$ from 4-8 whole cell experiments. The result suggests a CX546 dependent leftward shift of apparent glutamate affinity. 
We addressed this issue by making a dose response curve for glutamate at various concentrations and found that the apparent affinity of glutamate increases from 10.7 $\mu \mathrm{M}$ in the absence of CX546 to $3.0 \mu \mathrm{M}$ in presence of CX546 (n=4-8, Figure 5, right panel).

\subsubsection{Additional evidences for the effect of CX546 on gating properties of nondesensitizing GluR1 receptors}

We tested the observations further by examining the receptor deactivation. Upon unbinding of agonist the receptor deactivation currents proceeds in the nondesensitizing mutant receptor with a single exponential time constant of $15.5 \pm 1.7$ ms $(n=6)$. The slowing of current deactivation in nondesensitizing mutant receptor (based on the removal of desensitization) is in contrast to wild-type receptors (SternBach et al., 1998). Upon coapplication of IDRA21 and CX516 the time constants remain unaffected (Figure 6C). On contrary, the deactivation time constants evoked by short $(2 \mathrm{~ms})$ or long pulses (1 s) at $10 \mathrm{mM}$ glutamate in the presence of CX546 was slowed by more than threefold (Figure 6A,B). A similar slowing of deactivation time constants was observed when we used a high affinity AMPA receptor agonist quisqualate (control:324 $\pm 44 \mathrm{~ms}, \mathrm{n}=5$; CX546: $693 \pm 37 \mathrm{~ms}, \mathrm{n}=3$ ). The onset time constants of currents evoked by application of $3 \mu \mathrm{M}$ quisqualate were unchanged by the application of CX546 (control: $36 \pm 3.1 \mathrm{~ms}, \mathrm{n}=5$; CX546: $39 \pm 1.7 \mathrm{~ms}, \mathrm{n}=6$ ). These results indicate that CX546 increases the apparent affinity of agonist in the nondesensitizing AMPA receptor mutant by slowing the unbinding kinetics of the
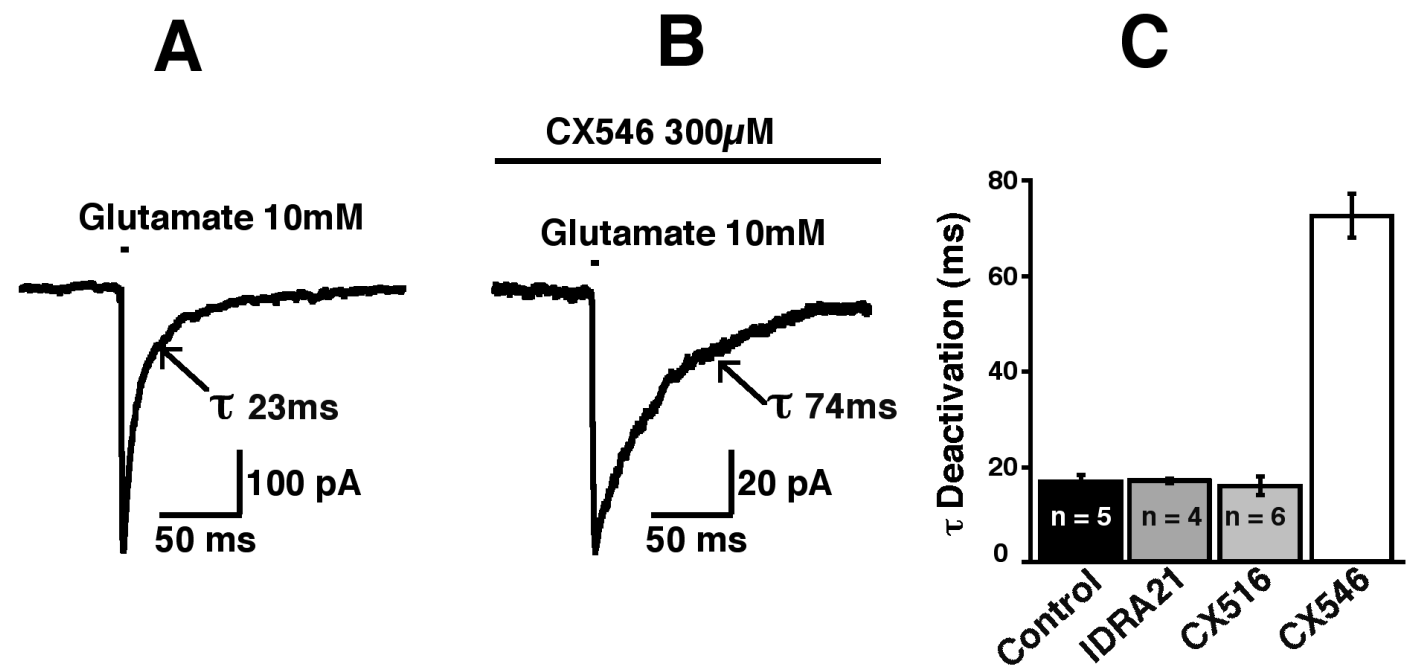
Figure 6. CX546 slows the deactivation of nondesensitizing GluR1 receptors

(A)Two typical responses of outside-out patches excised from HEK cells expressing R1L497Y being exposed to $2 \mathrm{~ms}$ pulses of $10 \mathrm{mM}$ glutamate in absence (A) and presence of CX546 (B). (C) Bar plot summarizing the mean \pm SE for deactivation time constants following the removal of agonist (1 s application, $10 \mathrm{mM}$ glutamate) from untreated R1L497Y patches (control), and modulator treated patches.

agonist.

\subsubsection{Specific binding properties of CX546}

It is known from previous studies that cyclothiazide (a potent blocker of desensitization) has an unusually slow on and offset kinetics for kainate-mediated currents in $\mathrm{R} 1_{\text {flip }}$ receptors (Partin et al., 1995; Partin et al., 1994). In molecular terms one may understand that cyclothiazide binds in a space at the interface of two neighboring subunits that are not readily accessible (Armstrong and Gouaux, 2000; Armstrong et al., 1998; Partin et al., 1994; Partin et al., 1993). This mechanism is employed by cyclothiazide to obtain efficient block of desensitization and avoid mediating the fast channel block (Partin et al., 1994). This can be observed by preincubating the patches with cyclothiazide before exposing them to agonist (see Figure 7A). In contrast to cyclothiazide, if CX546 is added to the control solution instead of agonist solution, the time constant of desensitization ( $\tau$ des $=8.0 \pm 0.5 \mathrm{~ms}$ $(n=6)$ and the amount of desensitization $(P / S=22.5 \pm 3.3 ; n=5$; Figure $7 B)$ is not modulated significantly. CX546 reaches full effectiveness of blocking desensitization in the agonist solution. This effect does not depend whether the patch is preincubated with CX546 or not (Figure 7C). 


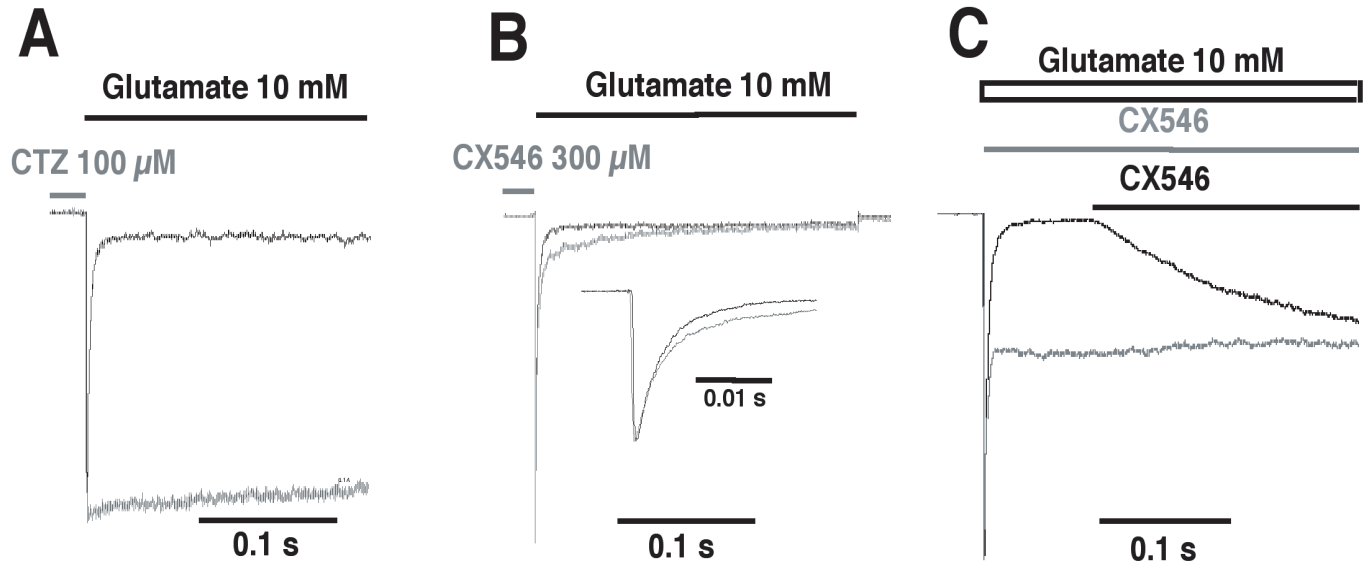

Figure 7. Kinetic state-dependent binding of AMPA modulators

(A) Glutamate $(10 \mathrm{mM})$ induced responses from an outside-out patch excised from HEK cells expressing $\mathrm{R} 2$ flip receptors. Preexposure of the patch to $100 \mu \mathrm{M}$ cyclothiazide is sufficient for the complete block of desensitization (grey trace). (B) Preincubation of CX546 $(300 \mu \mathrm{M})$ alone shows weak modulator effects as shown in another outside-out patch. Onset of current response is shown in the insert on an expanded time scale. (C) Two normalized current traces evoked by the application of $10 \mathrm{mM}$ glutamate are superimposed. The gray trace shows rapid removal of desensitization when CX546 is coapplied with the agonist, but removal of desensitization is greatly slowed when CX546 is coapplied when the response had fully desensitized (black trace). Data are from two different patches.

These results show differential mechanistic feature adopted by CX546 and cyclothiazide for the modulation of desensitization. The observations imply that CX546 may only bind to the receptor if the agonist is present or in an alternative way the binding/unbinding kinetics of CX546 is extremely rapid.

\subsubsection{Determining the binding properties of CX546 to AMPA receptors}

\subsection{Kinetics of binding in the presence of saturating concentration of glutamate}

We measured the kinetics of potentiation for on- and offset during a long application (2-4 s) of saturating concentration of glutamate $(10 \mathrm{mM})$ to examine if 
CX546 would bind to the receptor only in the presence of agonist on flip version of R1 and R2 subunits (Figure 7C, 8A, B). A pulse of 100-200 ms following the onset of glutamate application led to a response that reached full desensitization. We then coapplied CX546 at a concentration of $300 \mu \mathrm{M}$. To our surprise, we observed that the response amplitude reached its new steady state amplitude with an unusual slow time constant of $172 \pm 12 \mathrm{~ms}(\mathrm{n}=7)$ for R1 (Figure 8A and 10B (section 3.11.3.1.3)) and $286 \pm 26 \mathrm{~ms}(\mathrm{n}=7)$ for R2 (Fig 8B, 10B). These results are in contrast to the rapid onset of block of desensitization if glutamate and CX546 is coapplied at the beginning of the response (Figure 2C (section 3.11.1.1) and Figure 7C (section3.11.3)). These results imply that CX546 is prevented from binding to the receptor when the receptor is in desensitized state. An alternative explanation would be that the binding of CX546 to the desensitized state is not effective in removing the desensitization. The onset of recovery from desensitization upon application of CX546 is most likely based on a slow intrinsic rate of the receptor out of the desensitized state.
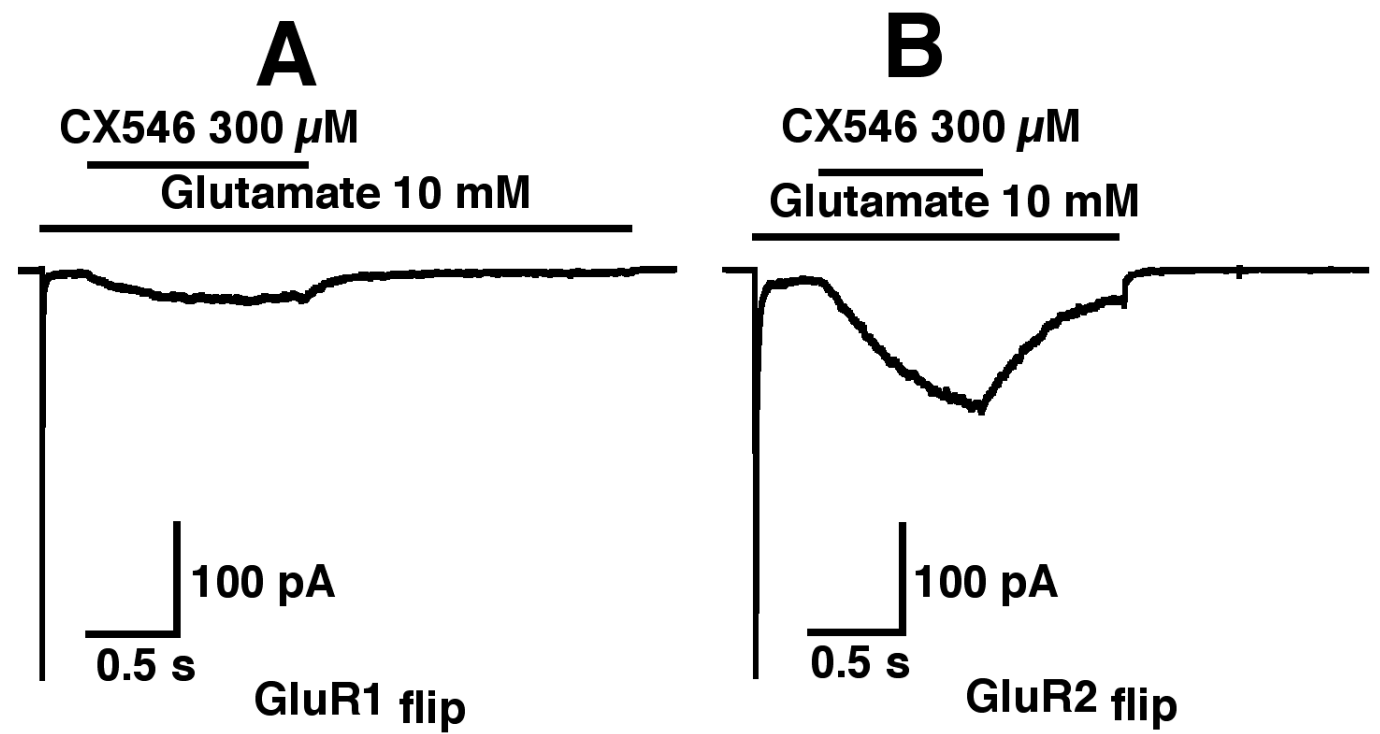

Figure 8. Onset and offset kinetics of CX546 induced removal of desensitization in GluR1 and GluR2 subunit

(A, B) The onset and offset kinetics of CX546 induced potentiation for R1(Q) (A) and $\mathrm{R} 2(\mathrm{Q})(\mathrm{B})$ receptors. Outside-out patch excised from $\mathrm{R} 1_{\text {flip }}$ and $\mathrm{R} 2$ flip receptors were first exposed to $10 \mathrm{mM}$ glutamate to attain desensitization before they were exposed to a long application (1s) of CX546 in the presence of glutamate $(10 \mathrm{mM})$. 


\subsection{Kinetics of binding in the presence of intermediate concentration of glutamate}

In order to test the hypothesis whether the on- and offset of recovery from desensitization upon application of CX546 based on slow intrinsic rate of the receptor out of the desensitized state, we repeated this experiment at an intermediate agonist concentration. The binding kinetics of CX546 can be studied well under these conditions because sufficient channel current in patch is still present due to activation by glutamate, while a considerable population of receptor remains unbound by agonist and thus will be in the nondesensitized state. On the basis of these predictions that the onset of the modulated response is proportional to the amount of newly activating nondesensitized receptors, the onset of the CX546 response was approximately 3.9 times faster $(73 \pm 4 \mathrm{~ms} ; \mathrm{n}=5)$ when $\mathrm{R} 2$ receptors were activated by $50 \mu \mathrm{M}$ glutamate (Figure 9).

Our result showed that the onset of CX546 is instantaneous if coapplied with saturating agonist concentration, is intermediate if applied when glutamate is present at an intermediate concentration, and is slowest when the receptors accumulate in the desensitized state.

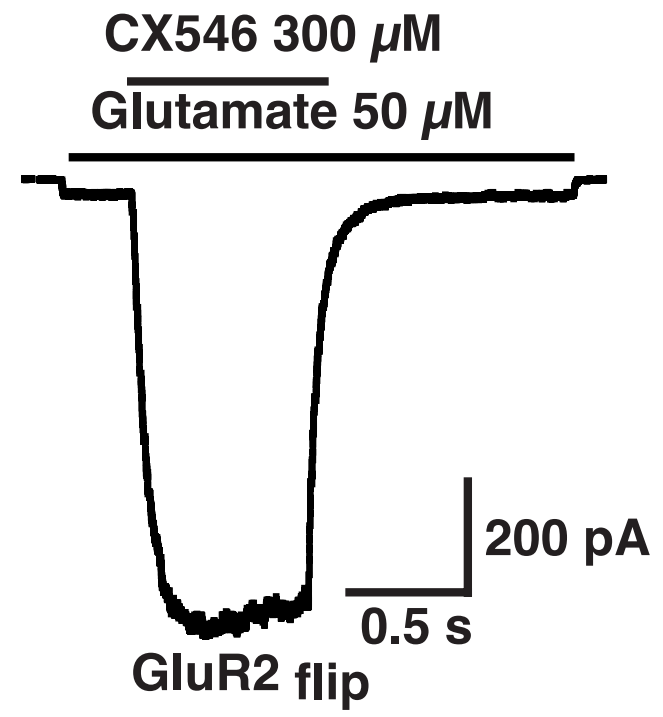

Figure 9. Increase in the rate of on- and offset kinetics of the receptors at intermediate concentration of agonist

Shown in the figure is the representative trace of the on- and offset kinetics of CX546 induced potentiation for $\mathrm{R} 2(\mathrm{Q})$ receptors at intermediate concentration of glutamate. Outside-out patch excised from $\mathrm{R} 2(\mathrm{Q})$ receptors were exposed to $50 \mu \mathrm{M}$ glutamate to allow a fraction of receptors to be in an agonist unbound state. On their exposure to CX546 containing agonist solution, the rate of on- and offset kinetics for the receptors 
got enhanced significantly.

\subsection{Analysis of the receptor kinetics for potentiation and recovery mediated by $C X 546$}

We analysed the kinetics of recovery from potentiation following the removal of CX546 using homomeric R2 flip receptors. This showed that the offset of current was faster at intermediate agonist concentrations $(73 \pm 4 \mathrm{~ms}, \mathrm{n}=5)$ than at saturating concentrations $(199 \pm 12 \mathrm{~ms}, \mathrm{n}=7)$ (Figure 9,10A). Furthermore we observed that the association and dissociation time constant (as determined by fitting exponential to onset of potentiation and recovery from potentiation) for R2 subunit is significantly slower than R1 subunit at saturating concentration of glutamate $(10 \mathrm{mM})$ (see Figure 10B). These results further support the hypothesis that the unbinding of agonist accelerates the unbinding kinetics of CX546.
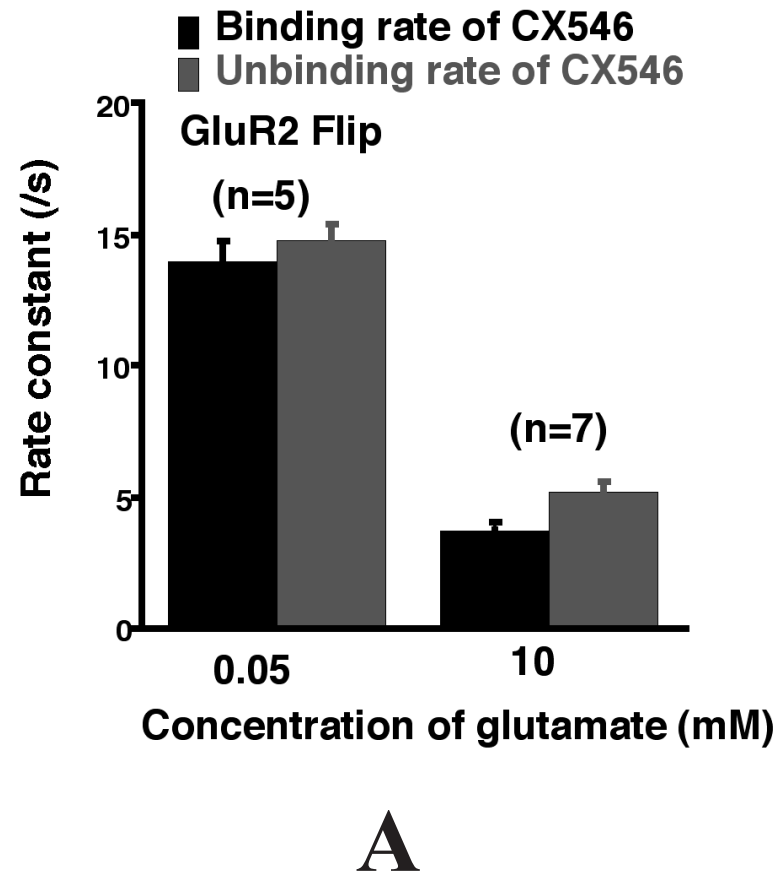

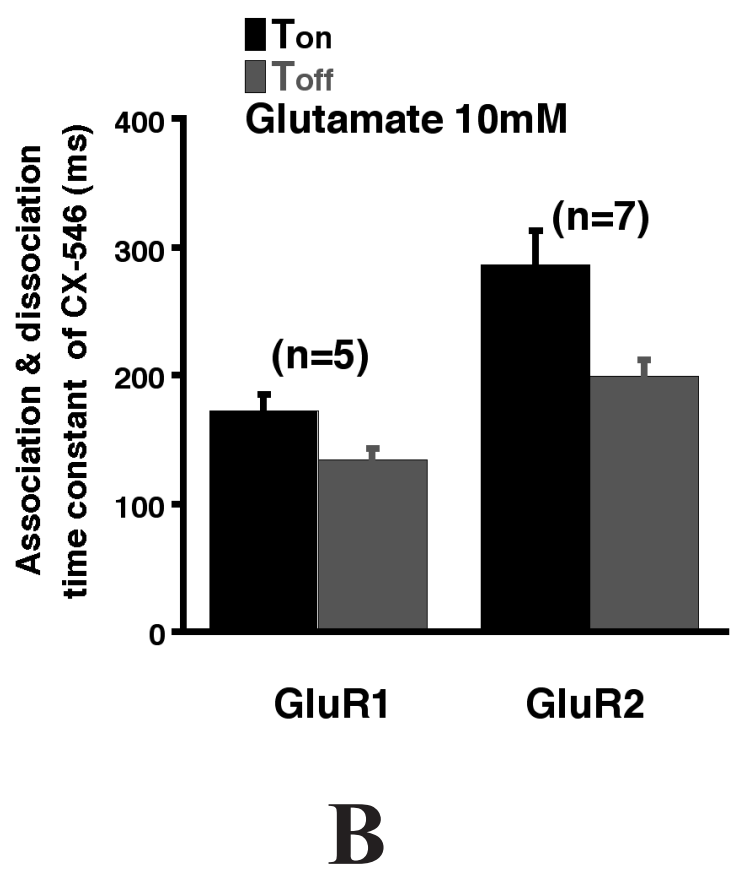

Figure 10. Analysis of binding and unbinding kinetics of CX546 at different concentrations of agonist

(A)The plot compares rate constant (1/on and 1/off) of CX546 induced onset rate of current potentiation and offset rate from potentiation of $\mathrm{R} 2$ receptors activated by 50 $\mu \mathrm{M}$ and $10 \mathrm{mM}$ glutamate. On- and offset rates are significantly faster at low agonist 
concentrations. (B) Comparison of on- and offset time constants from GluR1 and GluR2 receptors induced by a 1s application of $300 \mu \mathrm{M}$ CX546 at saturating concentration of glutamate $(10 \mathrm{mM})$.

\subsubsection{Comparison of the binding properties of CX546 with other AMPA receptor modulators}

We compared the unique binding properties CX546 with the AMPA modulator IDRA 21. The onset of the modulator response under similar experimental condition was much faster for IDRA21 at both low $(22 \pm 1 \mathrm{~ms}, \mathrm{n}=6)$ and saturating concentration of agonist $(49 \pm 14 \mathrm{~ms}, \mathrm{n}=5$ ) (Figure 11A, 11B). This suggests that IDRA21 is not as conformationally restricted in binding to the receptor as compared to CX546. We however observed that binding at lower agonist concentration was still 2.2 times faster that at saturating concentrations. A significant second slow on- $(212 \pm$ $33 \mathrm{~ms}$ ) and offset (456 $\pm 62 \mathrm{~ms})$ time constant for IDRA21 was observed during the onset and recovery from potentiation (data not shown). This may imply a more complex mechanism of IDRA21 in modulating AMPA-receptor gating. These data suggest that IDRA21 binds to the desensitized state of the receptor but with lower efficacy than to the nondesensitized state.

A

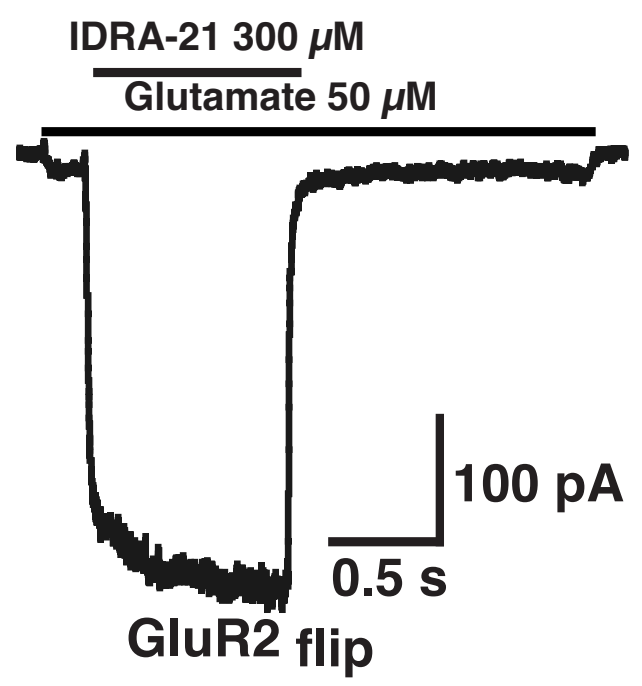

B
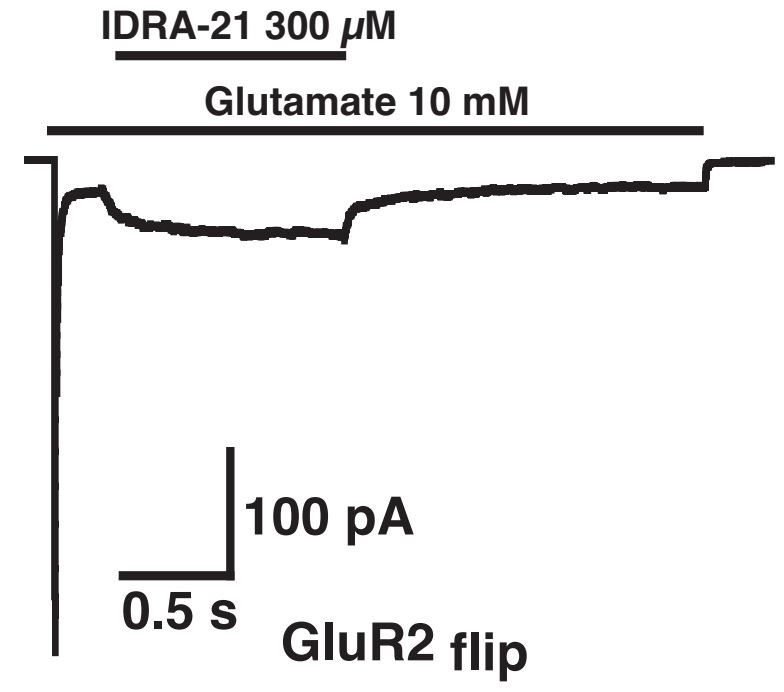

Figure 11. Effect of IDRA-21 on GluR2 flip receptors at different concentrations of agonist 
Outside-out patches excised from HEK 293 cells expressing $\mathrm{R} 2_{\text {flip }}$ receptors were exposed to half-maximal $(50 \mu \mathrm{M})(\mathrm{A})$ and saturating $(10 \mathrm{mM})$ concentration of glutamate (B). Note that the rate of on- and offset kinetics of receptors increases at low concentration of glutamate $(50 \mu \mathrm{M})(\mathrm{A})$.

\subsubsection{Effect of modulators on native receptors}

We studied the effect of IDRA21 and ampakines (CX516 \& CX546) on native receptors by excising outside-out patches from cultured mouse hippocampal neurons. We used 7-14 days old cells in culture for the experiments . The exemplary traces of glutamate evoked responses in the absence (control) and presence of modulators are shown in Figure 12A. The concentration of modulators were set to 300 $\mu \mathrm{M}$ in all experiments. We observed cell-to-cell variability during the data analysis. The possible reasons may be variable receptor subunit composition and splice variant isoforms in the neurons. The efficacy of blocking desensitization $(\mathrm{P} / \mathrm{S})$ for different modulators followed the same rank order CX546>IDRA21 $>$ CX516 (Figure 12, B1) as compared to recombinant R2 receptors (Figure 2B). All the drugs tested showed a significantly higher efficacy in modulating desensitization as compared to their effect on homomeric $\mathrm{R} 1 / \mathrm{R} 2_{\text {flip }}$ receptors. The amount of desensitization $(\mathrm{P} / \mathrm{S})$ under control conditions $(34 \pm 3(\mathrm{n}=21))$ was reduced to $1.76 \pm 0.15(\mathrm{n}=7)$ in the presence of CX546. We observed an approximately 30\% remaining desensitizing component in the presence of CX546 which desensitized with a single rapid, wildtype-like time constant of $2.8 \pm 0.2 \mathrm{~ms}(\mathrm{n}=7)$. The remaining fast time constant of desensitization seen in the other modulators is due to a receptor population that is either not saturated with the modulator or are not affected by them.

In the presence of IDRA 21 and CX516 the amount of desensitization was reduced to $2.22 \pm 0.35(n=6)$ and $8 \pm 0.8(n=5)$ respectively. In the presence of IDRA21 we observed at least two time constants of desensitization. The remaining desensitizing component in IDRA21 decayed with two time constant of $\tau_{\text {fast }}$ $($ IDRA21 $)=2.80 \pm 0.38 \mathrm{~ms}(54 \pm 19 \%), \tau_{\text {slow }}($ IDRA21 $)=87 \pm 45 \mathrm{~ms}(26 \pm 4 \%)$ $(n=4)$. In the presence of CX516 the desensitization time constant was best described with the sum of two exponentials of $\tau_{\text {fast }}=16 \pm 1.8 \mathrm{~ms}(51 \pm 2 \%), \tau_{\text {slow }}=139 \pm 3 \mathrm{~ms}$ $(28 \pm 2 \%)$. 

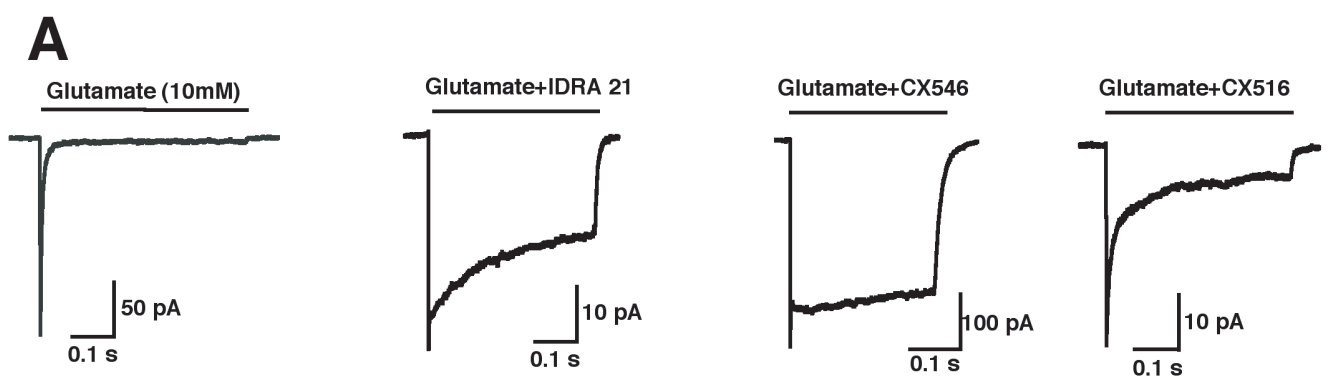

Control
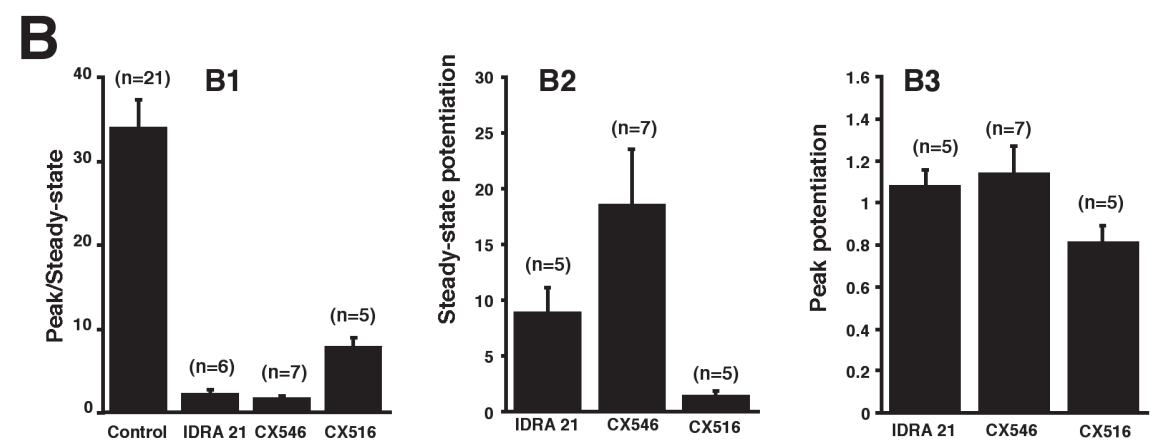

Figure 12. Effects of AMPA modulators on native receptors

(A) Currents evoked by long (1 s) application of $10 \mathrm{mM}$ glutamate in the absence (control) and presence of IDRA21, CX546, and CX516 at $300 \mu \mathrm{M}$. Data are represented from different outside-out patch measurements. We used D-AP5 (50 $\mu \mathrm{M})$ in extracellular solution to prevent NMDA mediated currents throughout the experiment. All modulators were mixed to agonist solution only. (B) Effect of modulators on the desensitization properties of synaptic receptors. (B1) Comparison of the effect of different modulators on the amount of desensitization $(\mathrm{P} / \mathrm{S})$. (B2) Potentiation of steady state currents upon application of modualtors (B3). Potentiation of peak amplitude upon application of modulators.

We tested finally the effect of AMPA modulators on the peak amplitudes of synaptic receptors. We observed that all the modulators tested did not affect the peak amplitudes of the response significantly. The amplitude ratio to the control amplitudes were $1.08 \pm 0.07(n=5), 1.14 \pm 0.12(n=7)$ and $1.02 \pm 0.01(n=4)$ for IDRA 21, CX546 and CX516 respectively (Figure 12B3). These result contrasts to the effect observed by the action of cyclothiazide (Partin et al., 1993) along with the recent observations made in cerebellar purkinje neurons, hippocampal neurons (Gates et al., 2001) and cortical neurons (Baumbarger et al., 2001). 


\subsubsection{Effect of modulators on synaptic transmission}

We have examined the modulatory effect of IDRA21 and ampakines (CX546 \& CX516) on amplitude and shape of synaptically activated excitatory currents. We used 9-14 days old cultured hippocampal neurons forming autapses on microisland cultures for our experiments. The concentration of modulator was set to $300 \mu \mathrm{M}$. Low frequency stimulation of $0.2 \mathrm{~Hz}$ evoked postsynaptic currents which decayed with time constant of $7.0 \pm 0.6 \mathrm{~ms}(\mathrm{n}=33)$ under control conditions (control). The EPSCs of control (black) and modulator responses (gray) are superimposed and shown in figure 13A. Application of IDRA 21, CX546 and CX516 not only enhanced the EPSC amplitude but also slowed down the decay time course. The EPSC decayed with a time constant of $8.8 \pm 0.5 \mathrm{~ms}(\mathrm{n}=10)$ in CX516, to $25 \pm 3 \mathrm{~ms}(\mathrm{n}=8)$, and to $23 \pm 1.2$ $(n=6)$ in the presence of cyclothiazide. The rise times $20-80 \%$ (not shown) was not significantly affected in the presence of any of the modulators tested. We then measured the net charge transfer of integrated currents and synaptic amplitude in the presence of modulators as shown in Figure 13B. The similarity in the enhancement of the EPSC total charge for CX546 and cyclothaizide imply that CX546 is as efficient as cyclothiazide in modulating the synaptic responses. The total charge was approximately 3.3- and 5.6-fold higher than for IDRA 21 and CX516 respectively. 

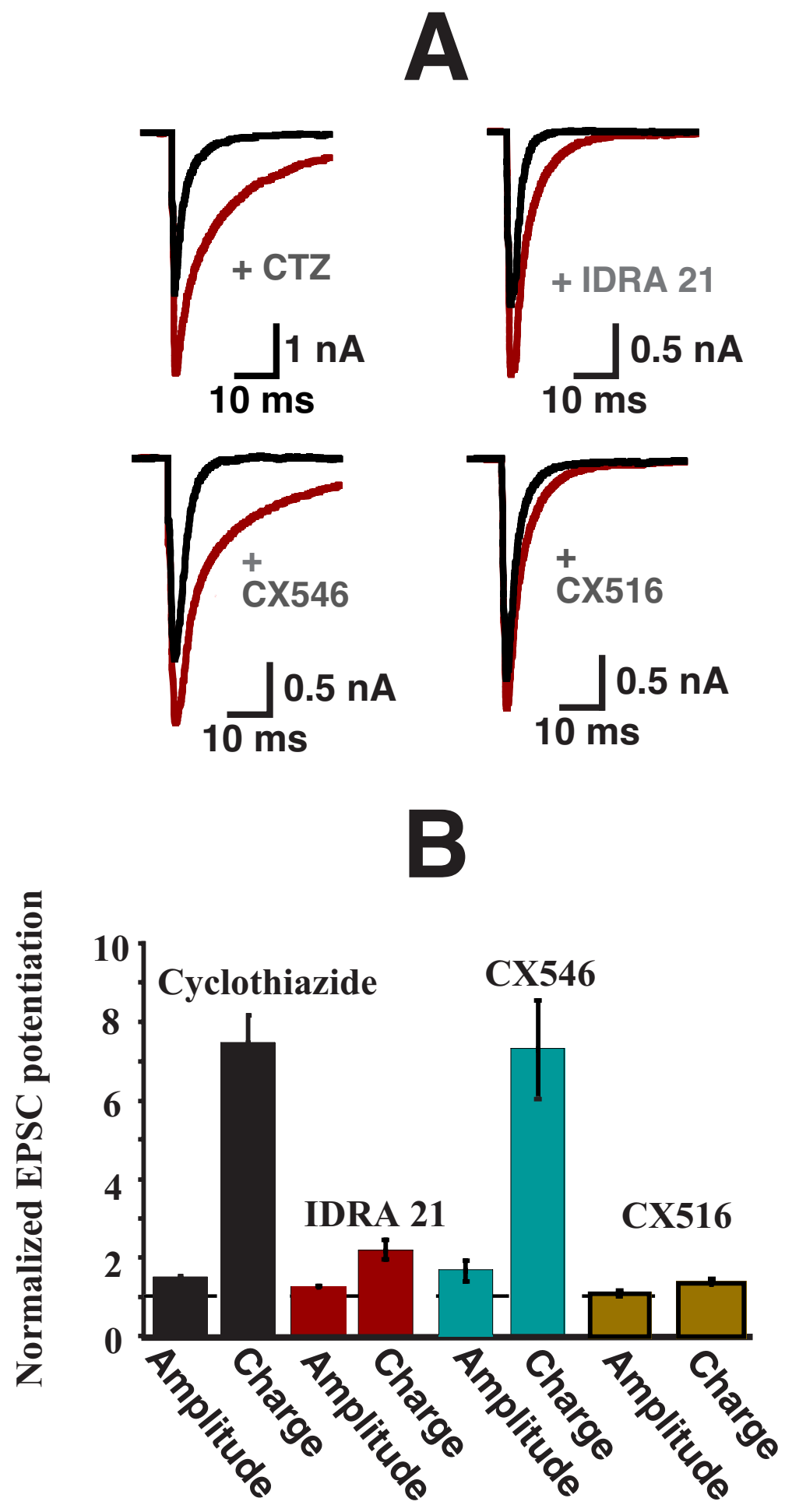

Figure 13. Effect of AMPA modulators on synaptic transmission

(A) Effect of cyclothiazide $(100 \mu \mathrm{M})$, IDRA 21, CX546 and CX516 at a concentration of $300 \mu \mathrm{M}$ on EPSC evoked at $0.2 \mathrm{~Hz}$ in autaptic hippocampal cultures. Representative EPSCs are overlaid in the absence (black trace) and presence (grey trace) of modulators. Current transients that resulted from the $2 \mathrm{~ms}$ depolarization to induce EPSC were blanked for display purposes (B) Bar plot representing the potentiating effect of modulators on the amplitude and net charge of EPSCs. The 
responses in the presence of modulators are normalized against the untreated EPSC. These recordings were performed by C.Rosenmund.

\subsubsection{Mimicking the gating properties of synaptic receptors by deactivation kinetics}

We examined short (2 ms) pulses of glutamate on patches containing GluR2Q receptors to mimic the effect of the modulator CX546 on the activation of AMPA receptors at the synapse (Koike et al., 2000). The deactivation of currents following the removal of agonist was slightly, but significantly slowed $\left(\tau_{\text {deactivation }}\right.$ control: $0.86 \pm 0.11 \mathrm{~ms}, \mathrm{n}=12$; CX546: $1.27 \pm 0.15, \mathrm{n}=11 ; \mathrm{p}=0.046)$. These were consistent with the previous findings using cyclothiazide on GluR1 subunit (Partin et al., 1996). The fast deactivation of GluR2 subunits in the presence of CX546 in comparison to approximately 20 fold slower time constant of decay for the synaptic current implies that the concentration of glutamate necessary to activate the postsynaptic AMPA receptor extends for several milliseconds (Barbour et al., 1994; Mennerick and Zorumski, 1994).

\section{Patch GluR2 Flip}

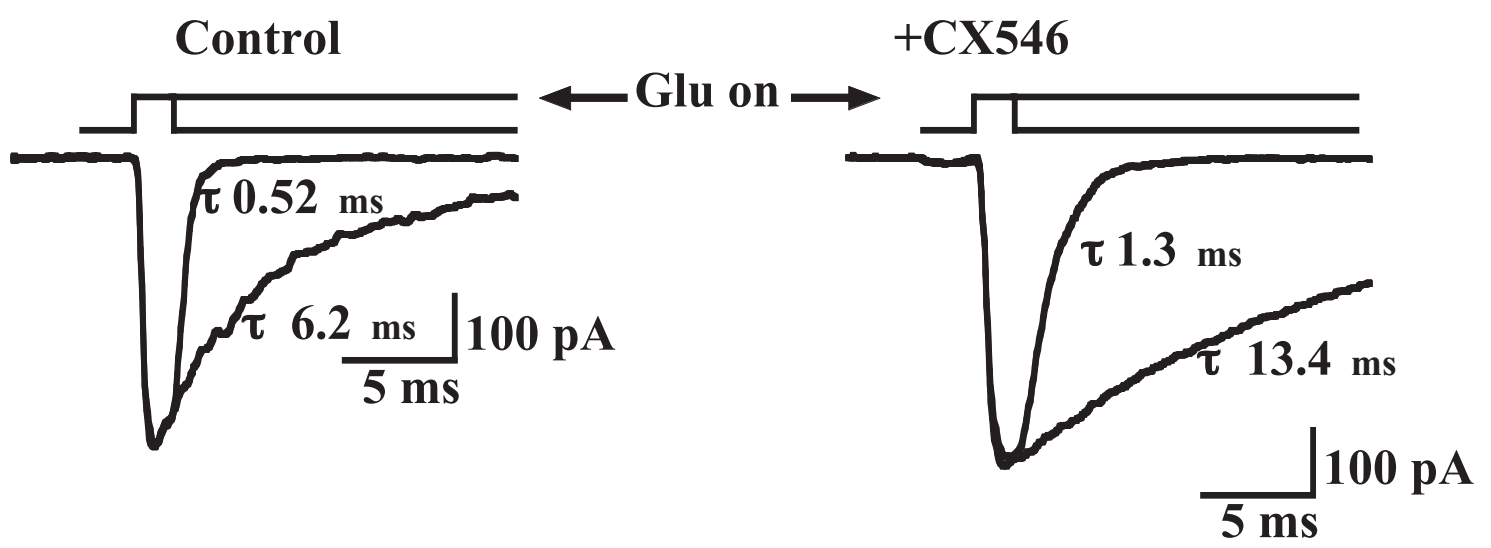

Figure 14. Comparing the deactivation versus desensitization kinetics in GluR2 receptors

Overlaid are the normalized traces of long pulse $(250 \mathrm{~ms})$ and short pulse $(2 \mathrm{~ms})$ of saturating concentration of glutamate $(10 \mathrm{mM})$ to distinguish desensitization and deactivation from a untreated R2(Q) patch (left) and a patch treated with $300 \mu \mathrm{M}$ CX546 (right). The deactivation was significantly but slightly increased. In presence of CX546, deactivation was in average 20-fold faster than the EPSC decay time constant. 
The differential deactivation kinetics observed here may not exclude the possibilities of variable subunit combination and stoichiometry as observed in synaptic AMPA receptors. The lack of desensitization and the increased affinity of the receptor for glutamate in the presence of modulators may allow the receptor to sense prolonged ambient glutamate concentrations in the synaptic cleft. This may be a possible reason for observing the slow decay of EPSC. Hence one can consider the possibility that in wild type synapses, both the low affinity for glutamate and desensitization may be determining factor to assure brief EPSCs, despite a prolonged presence of glutamate in the synaptic cleft (Barbour et al., 1994; Diamond and Jahr, 1995; Mennerick and Zorumski, 1994; Raman and Trussell, 1995).

\subsection{Studies on the splice variants of kainate receptors}

In the previous chapters we discussed the gating and assembly properties of AMPA receptors. In this chapter we study another main group of ionotropic glutamate receptors called kainate receptors. These receptors can be activated specifically by the agonist kainate. The importance of kainate receptors in excitatory synaptic transmission is poorly understood, although their role in metabotropic functions has been implicated in several studies (Kullmann, 2001). Furthermore, they play important role in modulating the release of GABA by presynaptic mechanisms and are thought to be involved in nociceptive (pain sensing) and thermoceptive sensory transmission (Li et al., 1999).

Kainate receptors differ from AMPA receptors in several aspects. Although the gating properties of these receptors are similar to AMPA receptors they resensitize significantly slower than AMPA receptors (Heckmann et al., 1996). Kainate receptors undergo alternative splicing (N-terminus and C-terminus) and RNA editing at sites that are different from AMPA receptors (see section 1.8.1 and 1.8.2). From previous studies it is known from the AMPA receptors that alternative splicing affects the gating properties (Sommer et al., 1990). Furthermore, it is not known whether the structural elements in these splicing regions are important for controlling the gating properties and assembly of kainate receptors. The main focus of our work was divided into two sub parts. In the first part, we were interested in understanding the importance of C-terminus of R6 subunit in controlling the surface expression and gating mechanism of the receptors. In the second part we study the functional and gating properties of different splice variants of R5 subunit. The receptors that are 
discussed in the section below were tagged by N-terminal myc epitope just before the signal sequence. The insertion of myc-epitope did not apparently affect the gating properties and degree of expression in these receptors (based on the results from characterization of gating parameters in myc-R6 subunit see table 1) as compared to their untagged isoforms described in previous reports (Lerma et al., 2001). The peak amplitudes of R6 subunit with myc-epitope was $1794 \pm 876$ pA (n=7).

\subsubsection{Role of C-terminus in controlling the gating and expression properties of GluR6 subunit}

The goal of this work was to study if the C-terminus region in R6 subunit is involved in controlling the gating properties of the receptors. Various role of this Cterminus region of R6 subunit has been described in previous studies. As for example, this region shows a strong association with PDZ1 domain of SAP-90, a postsynaptic density protein (PSD) found in central synapses (Piserchio et al., 2002) while a week association with other PSD proteins like SAP 97 (Mehta et al., 2001). It has been reported that the association of postsynaptic density proteins slow down the desensitization process (Garcia et al., 1998).

To understand the role of C-terminus of R6 subunit, deletion mutants were constructed. These mutants varied in the length of C-terminus from R6 subunit. The mutants were named R6-4, R6-14, and R6-54 for the deletion of last 4, 14 and 54 amino acids in the C-terminus of R6 subunit respectively.

The gating properties of C-terminal deletion mutants of GluR6 subunit were characterized by determining the four gating parameters (1) time constant of desensitization, (2) amount of desensitization (Peak/Steady-state, (P/S ratio)), (3) resensitization and (4) deactivation kinetics. We studied these properties both in wild type and C-terminal deletion mutants of R6 receptors. The gating properties were studied in the presence of glutamate and kainate at saturating concentrations. The experimental conditions were maintained same as described for AMPA receptors.

All the receptors were functional although the level expression as assessed by the rate of success in the functional responses and the measured average amplitudes varied. First we measured the glutamate $(10 \mathrm{mM})$ responses from wild-type R6subunit (the Q and R forms). Application of 10mM glutamate to either R6(Q) or R6(R) elicited a fast activation followed by desensitizing current of time course 4-5 ms ( $n=7$ for R6(Q) and $n=12$ for R6(R) (see table 1). We observed that the kinetics (the 
time constant of desensitization) of desensitization and amount of desensitization (P/S ratio) was not significantly different between $\mathrm{Q}$ and $\mathrm{R}$ forms of R6 subunit (Fig 1A, $\mathrm{B}$, table 1). However we observed as expected, the reduction in peak amplitude for R6(R) subunit by 12.8 fold as compared to R6(Q) subunit (See table 1). These results are consistent with the previous observations (Paternain et al., 2000). Furthermore, we measured the deactivation kinetics by exposing the outside-out patches to short pulses $(2 \mathrm{~ms})$ of glutamate $(10 \mathrm{mM})$. Short pulses of glutamate $(10 \mathrm{mM})$ elicited a response that decayed with time constant of $2.57 \pm 0.09 \mathrm{~ms}$ in R6(Q) subunit ( $\mathrm{n}=7$ ), while with time constant of $3.9 \pm 0.37 \mathrm{~ms}(\mathrm{n}=5)$ for R6(R) subunit. These results showed that the deactivation of receptors proceeds faster than desensitization by 1.7 fold (see Figure 1C, Table 1). The time constant of deactivation in R6(Q) subunit is slower by more than three-fold in comparison to the previous reported values for deactivation time constant in AMPA receptors (Partin et al., 1996). Furthermore, to determine the recovery from desensitization (resensitization) we used the standard paired pulse experiments. Representative traces of paired pulse experiment obtained from R6(Q) subunit are shown in figure 1D. However we did not observe any significant difference in the rate of resensitization between $\mathrm{Q}$ and $\mathrm{R}$ forms of R6 subunit. 


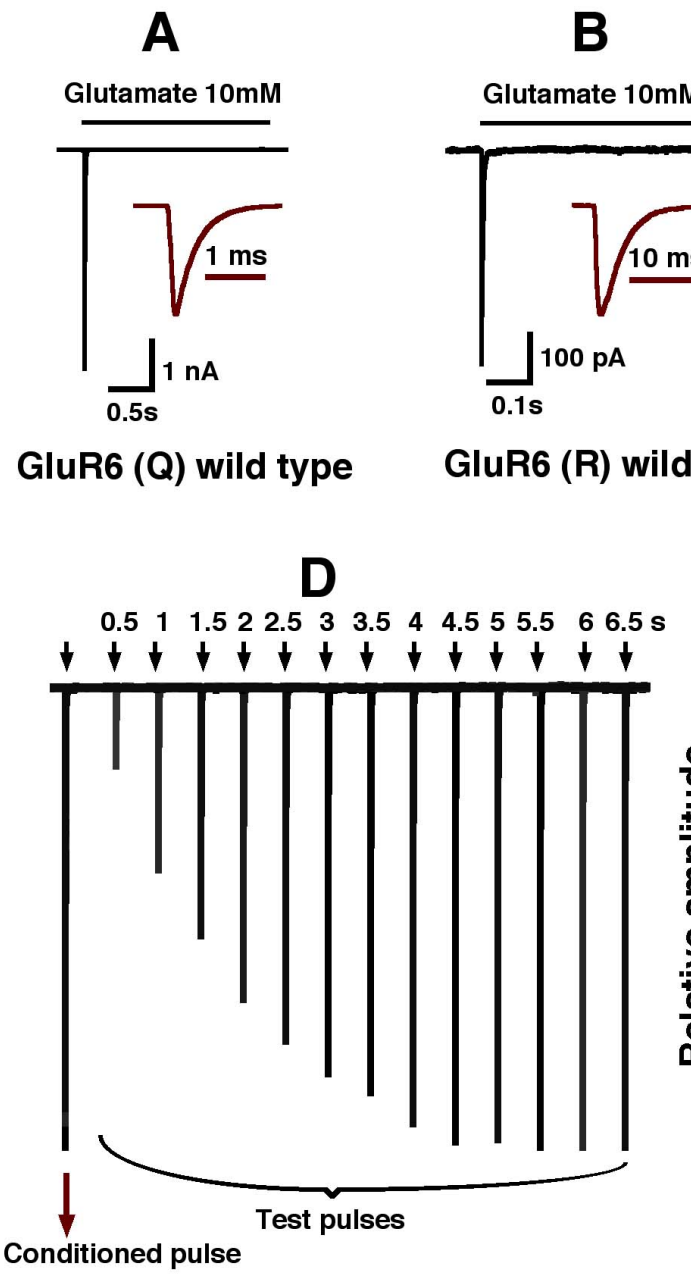

GluR6 (Q) wild type

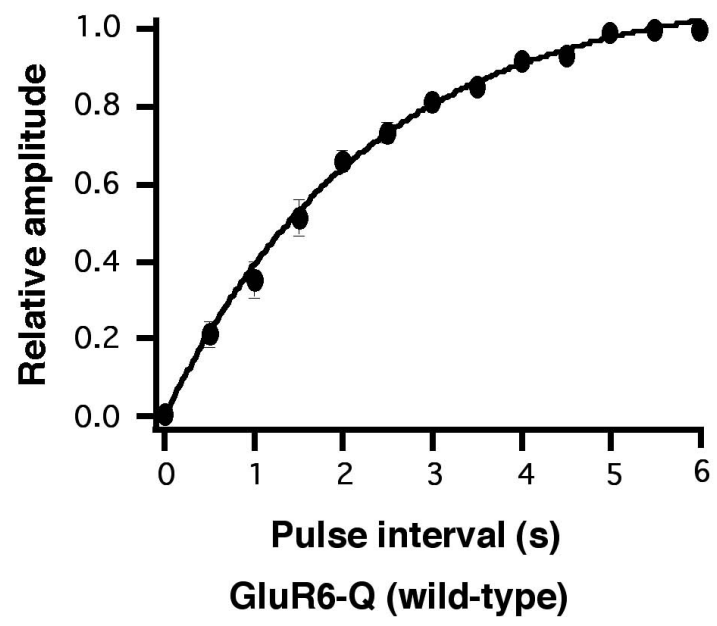

Figure 1. Kinetics of myc-epitope tagged GluR6 subunit

(A, B) Typical traces of outside-out patches from HEK 293 cells expressing R6 (Q) and R6(R) subunit in the presence of $10 \mathrm{mM}$ glutamate. Inset shows the trace on an expanded time scale. (C) Overlaid are the normalized traces of long pulse $(250 \mathrm{~ms})$ and short pulse $(2 \mathrm{~ms})$ of saturating concentration of glutamate $(10 \mathrm{mM})$ to distinguish desensitization and deactivation of R6(Q) subunit. (D) Overlaid are the 14 normalized traces recorded from a single cell by applying paired pulses of saturating concentration of glutamate $(10 \mathrm{mM})$. A $50 \mathrm{~ms}$ conditioning pulse was applied followed by test pulses at different time intervals to determine the recovery from desensitization. Traces were normalized to correct for the rundown. The ratio of the peak amplitude of the test response to the conditioning response was measured to determine the relative amplitude (I2/I1, mean $\pm \mathrm{SEM}$ ). The relative amplitude was plotted against pulse intervals (s) and exponential fits were performed to determine the rate of resensitization (E). A single exponential fit showing the recovery from desensitization. The relative amplitude (I2/I1) ratio of conditioning pulse to test pulse 
is plotted against pulse intervals. Data were collected from 7 cells and pooled to determine the recovery at specific pulse duration.

\begin{tabular}{|c|c|c|c|c|c|c|}
\hline & \multicolumn{6}{|c|}{$\begin{array}{l}\text { Summary of biophysical parameters of wild-type and } \\
\text { C-terminal deletion mutants of GluR6 subunit in the } \\
\text { presence of saturating concentration of glutamate }\end{array}$} \\
\hline Receptor & $\begin{array}{c}20-80 \% \text { Trise } \\
\text { ms }\end{array}$ & Tdes (ms) & $\begin{array}{l}\text { Tdeact } \\
\text { (ms) }\end{array}$ & I peak $(\mathrm{pA})$ & Peak/SS & $\begin{array}{c}\text { Res Rate } \\
\text { / S }\end{array}$ \\
\hline WT R6(Q) & $\begin{array}{c}0.70 \pm 0.13 \\
\quad(n=5)\end{array}$ & $\begin{array}{l}4.36 \pm 0.3 \\
(n=6)\end{array}$ & $\begin{array}{l}2.57 \pm 0.09 \\
(n=7)\end{array}$ & $\begin{array}{l}1794 \pm 876 \\
(n=7)\end{array}$ & $\begin{array}{c}262 \pm 63 \\
\quad(n=5)\end{array}$ & $\begin{array}{l}0.43 \pm 0.02 \\
(n=7)\end{array}$ \\
\hline WT R6(R) & $\begin{array}{l}0.57 \pm 0.02 \\
\quad(n=9)\end{array}$ & $\begin{array}{l}5.2 \pm 0.16 \\
\quad(n=9)\end{array}$ & $\begin{array}{l}3.9 \pm 0.37 \\
(n=5)\end{array}$ & $\begin{array}{l}140 \pm 40 \\
(n=12)\end{array}$ & $\begin{array}{l}294 \pm 38 \\
(n=10)\end{array}$ & $\begin{array}{l}0.38 \pm 0.03 \\
(n=6)\end{array}$ \\
\hline R6-4 & $\begin{array}{l}0.58 \pm 0.05 \\
(n=5)\end{array}$ & $\begin{array}{l}4.42 \pm 0.2 \\
(n=9)\end{array}$ & $\begin{array}{l}2.92 \pm 0.2 \\
(n=10)\end{array}$ & $\begin{array}{c}1560 \pm 467 \\
(n=12)\end{array}$ & $\begin{array}{l}246 \pm 37 \\
(n=8)\end{array}$ & $\begin{array}{c}0.36 \pm 0.02 \\
(n=10)\end{array}$ \\
\hline R6-14 & $\begin{array}{c}0.35 \pm 0.02 \\
(n=5)\end{array}$ & $\begin{array}{c}4.33 \pm 0.3 \\
(n=7)\end{array}$ & $\begin{array}{c}2.62 \pm 0.2 \\
(n=7)\end{array}$ & $\begin{array}{c}1019 \pm 301 \\
(n=7)\end{array}$ & $\begin{array}{c}215 \pm 52 \\
(n=5)\end{array}$ & $\begin{array}{c}0.55 \pm 0.04 \\
(n=6)\end{array}$ \\
\hline R6-54 & $\begin{array}{c}0.79 \pm 0.14 \\
\quad(n=5)\end{array}$ & $\begin{array}{c}3.46 \pm 0.4 \\
(n=3)\end{array}$ & $\begin{array}{c}2.6 \pm 0.3 \\
\quad(n=5)\end{array}$ & $\begin{array}{c}286 \pm 185 \\
(n=3)\end{array}$ & $\begin{array}{c}293 \pm 1.4 \\
(n=2)\end{array}$ & $\begin{array}{c}0.4 \pm 0.05 \\
(n=4)\end{array}$ \\
\hline
\end{tabular}

\section{Table 1}

Summary of the biophysical parameters of wild type and C-terminal deletion mutants of R6 receptors. Outside-out patches were excised from HEK 293 cells and were exposed to saturating concentration of glutamate $(10 \mathrm{mM}) . \mathrm{n}$ represents the number of cells used for the experiments.

Following similar procedures for determining the parameters of gating kinetics, we characterized the biophysical properties of C-terminal deletion mutants of R6 subunits. Essentially we did not observe any significant effect on the gating properties by deletion of C-terminus suggesting that this region does not play an important role in controlling the gating kinetics of the receptor.

Outside-out patches exposed to saturating concentration of glutamate (10 mM) resulted in activation of the receptors with a rise time $(20-80 \%)$ of $0.17 \pm 0.13 \mathrm{~ms}$ $(\mathrm{n}=5)$ for wild type R6 receptors, while it was $0.58 \pm 0.05 \mathrm{~ms}(\mathrm{n}=10)$ for $\mathrm{R} 6-4$, $0.35 \pm 0.02 \mathrm{~ms}(\mathrm{n}=7)$ for $\mathrm{R} 6-14$ and $0.79 \pm 0.14 \mathrm{~ms}(\mathrm{n}=3)$ for R6-54 respectively (see table 1). 
Furthermore, the analysis of time constant of desensitization, rate of resensitization and the time course of deactivation for the deletion mutants (GluR6-4, $-14,-54)$ did not show any significant difference in comparison to wild-type receptors by application of glutamate $(10 \mathrm{mM})$ (as shown, table 1). Surprisingly, peak amplitude of the deletion mutant R6-54 receptors $(286 \pm 186 ; n=3)$ was reduced by 6.2 fold as compared to wild-type $\mathrm{R} 6$ receptors $(1794 \pm 876 \mathrm{pA} ; \mathrm{n}=7)$. We noticed that the rate of successful responses in R6-54 receptors were relatively lesser than wild-type receptors. Only $10 \%$ of the patched cells were responsive while others did not respond for the application of glutamate. By comparing the measured responses of the average amplitudes from R6-54 mutant and wild-type receptors, we may overestimate the analysis of our work concerning the average amplitude of the responses. The possible speculations include improper insertion of the receptors into the membrane or destructions in trafficking of the receptors between endoplasmic reticulum and plasma membrane. Biochemical techniques like immunfluorescence can determine and further explain the exact solution for the poor expression.

\subsubsection{Characterization of gating properties of splice variants of GluR5 subunit}

GluR5 subunit occurs in three different alternatively spliced variant isoforms GluR5-1 and GluR5-2 (Lerma et al., 2001). GluR5-1 has an extra cassette of 15amino acids in the N-terminal region whereas compared to GluR5-2. This 15 amino acid insert in GluR5-1 is located just outside the S1 domain of the first putative transmemberane region. The S1 and S2 domains are important determinants for the agonist binding since the residues in these domains form the binding pocket for the agonist. This has been furthermore well elucidated with the help of X-ray crystallographic studies (Armstrong and Gouaux, 2000). GluR5-2 subunit in turn is spliced into three isoforms GluR5-2a, 5-2b and 5-2c. GluR5-2a is 49 amino acids shorter than GluR5-2b because of the introduction of premature stop codon. GluR5-2c contain an extra in-frame exon that makes it 29 amino acids longer than GluR5-2b. The so far characterized splice variant of GluR5-1 subunit represents the C-terminus sequence similar to that in GluR5-2b isoform. We analysed the biophysical properties of splice variants of GluR5 subunit (GluR5-A and GluR5-B isoforms) in this work. Furthermore we show that the deletion of 15- amino acid cassette affects the gating 
properties in GluR5 isoforms. The basic techniques and characterization were done similar to that for GluR6 subunit.

\subsubsection{Characterization of homomeric splice variants of GluR5 isoforms}

3.12.2.1.1 The isoforms of GluR5 splice variants and their $N$-terminal deletion are functional

The peak amplitude of the responses from all the splice variants of R5 subunits was less than R6 subunit by two orders of magnitude in outside out patch measurements. The low responsiveness of peak amplitude in R5 subunits limits our study to further characterize their kinetic properties. To obtain the higher amplitudes from several channels (that allows us to study the gating parameters), we utilized the whole cell configuration. The important limitations in using this configuration for electrical recording from HEK 293 cells are that (1) the fast solution exchange may

A

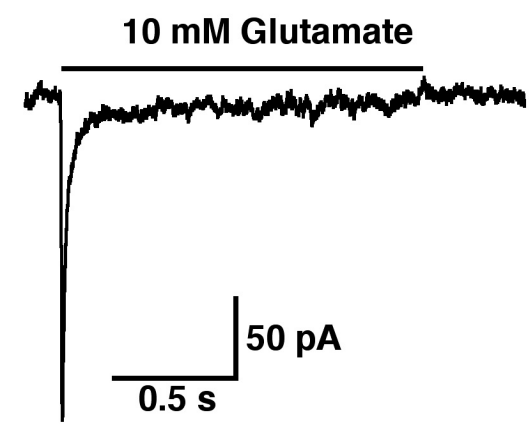

R5A-myc

C

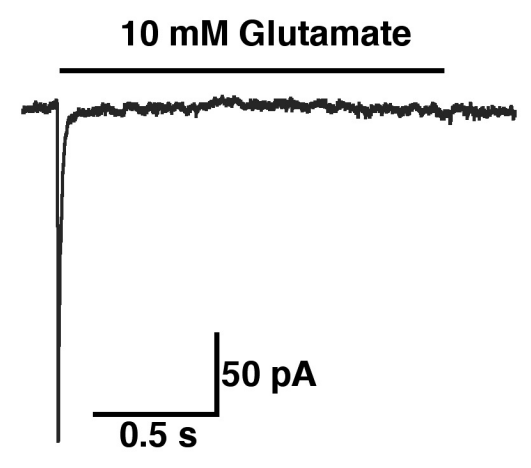

R5B-myc
B

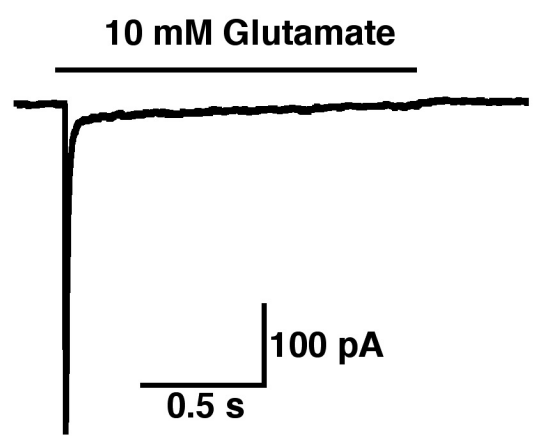

R5A (deletion)-myc

D

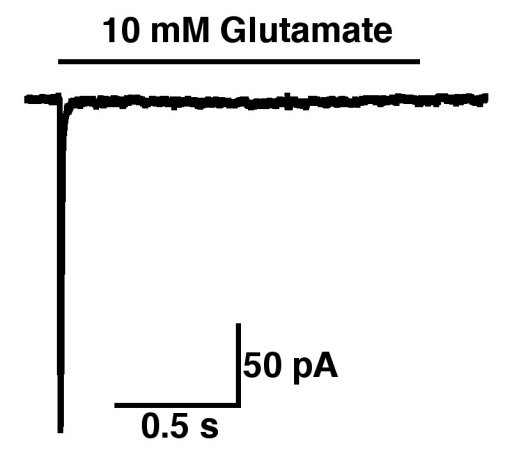

R5B (deletion)-myc

Figure 2. Desensitization in myc epitope tagged isoforms of GluR5 subunit and their deletion mutants 
(A, B, C, D) R5A and R5B receptors and their 15 amino acids deletion mutants (R5A (deletion)-myc and R5B (deletion)-myc) were expressed in HEK 293 cells. Whole cell recordings were performed on the cells expressing these isoforms. The currents were measured at holding potential of $-60 \mathrm{mV}$ in the presence of saturating concentration of glutamate $(10 \mathrm{mM})$.

not be obtained as compared to outside-out patch measurements (2) the solution exchange rate may be slower than the rate of desensitization in whole cell measurements

Whole cell recordings were first performed to know the level of expression and functional properties of myc-epitope tagged R5A, R5B, R5A (deletion), R5B (deletion) isoforms. Application of $10 \mathrm{mM}$ glutamate to whole cell from these receptors resulted in an inward current that had fast desensitization (Figure 2). In whole cell configuration we observed that the peak amplitudes of responses from GluR5 isoforms were 3-5 fold larger ( $\mathrm{n}=13$ for $\mathrm{R} 5 \mathrm{~A}$ and $\mathrm{n}=12$ for $\mathrm{R} 5 \mathrm{~B}$ ) than the responses from outside out patch measurements $(n=6-7)$ for saturating concentration of glutamate $(10 \mathrm{mM})$. Following the whole cell configuration, we observed that R5B isoforms elicited a much bigger inward currents (Peak amplitude $=573 \pm 242 \mathrm{pA}, \mathrm{n}=12$ ) than R5A isoforms (Peak amplitude $=318 \pm 102 \mathrm{pA}, \mathrm{n}=13$ ) suggesting that the insertion of $\mathrm{R} 5 \mathrm{~B}$ receptors into membrane is more efficient than $\mathrm{R} 5 \mathrm{~A}$. Furthermore, the deletion of 15 amino acids just outside the S1 domain from these isoforms did not affect the peak responsiveness in comparison to R5 isoforms when measured from whole cell recordings (Peak amplitude for R5A-del= 297 \pm 124 pA, $n=13$; R5B-del= $227 \pm 65 \mathrm{pA}, \mathrm{n}=11)$. These results imply that the removal of 15 amino acids just outside the S1 domain does not affect the overall responsiveness of the receptors.

\subsection{Effect of $N$-terminal deletion on gating properties of GluR5 isoforms}

\subsection{Effect on the rise time and time constant of desensitization}

Whole cell recordings were carried out to determine the basic gating properties of both splice variants and the deletion mutants of R5 subunit. We first measured rise time $(20-80 \%)$ in these isoforms. Analysis of the rise time is representative for the quality of solution exchange. Although not significantly, the deletion mutant of R5A (R5A-del) or R5B (R5B-del) isoforms were activated with a faster rise time (20-80\%) (R5A-del, Trise $=1.0 \pm 0.07 \mathrm{~ms}, \mathrm{n}=6$; R5B-del, Trise $=1.0 \pm 0.03 \mathrm{~ms}, \mathrm{n}=7$ ) as compared 
to wild type isoform of R5A or R5B (Trise $=1.3 \pm 0.1 \mathrm{~ms}, \mathrm{n}=13$ for $\mathrm{R} 5 \mathrm{~A}$ and $1.4 \pm 0.13$, $\mathrm{n}=13$ for $\mathrm{R} 5 \mathrm{~B}$ ) respectively (see figure $3 \mathrm{~A}$ ).

To know if other gating parameters are affected by deletion of 15 amino acid cassette we looked at the desensitizing phase in R5A and R5B isoforms. We did not observe any significant change in the time constant of desensitization between R5A and $\mathrm{R} 5 \mathrm{~B}$ isoforms upon activation by glutamate $(10 \mathrm{mM})$ (see figure $3 \mathrm{~B})$. However, surprisingly we observed that the deletion mutants of GluR5A and GluR5B isoforms desensitized nearly two fold faster than the non-deleted isoforms (as shown in figure 3B). These results suggest that the presence of 15 amino acids outside the $\mathrm{S} 1$ domain is important for controlling the rate of desensitization.
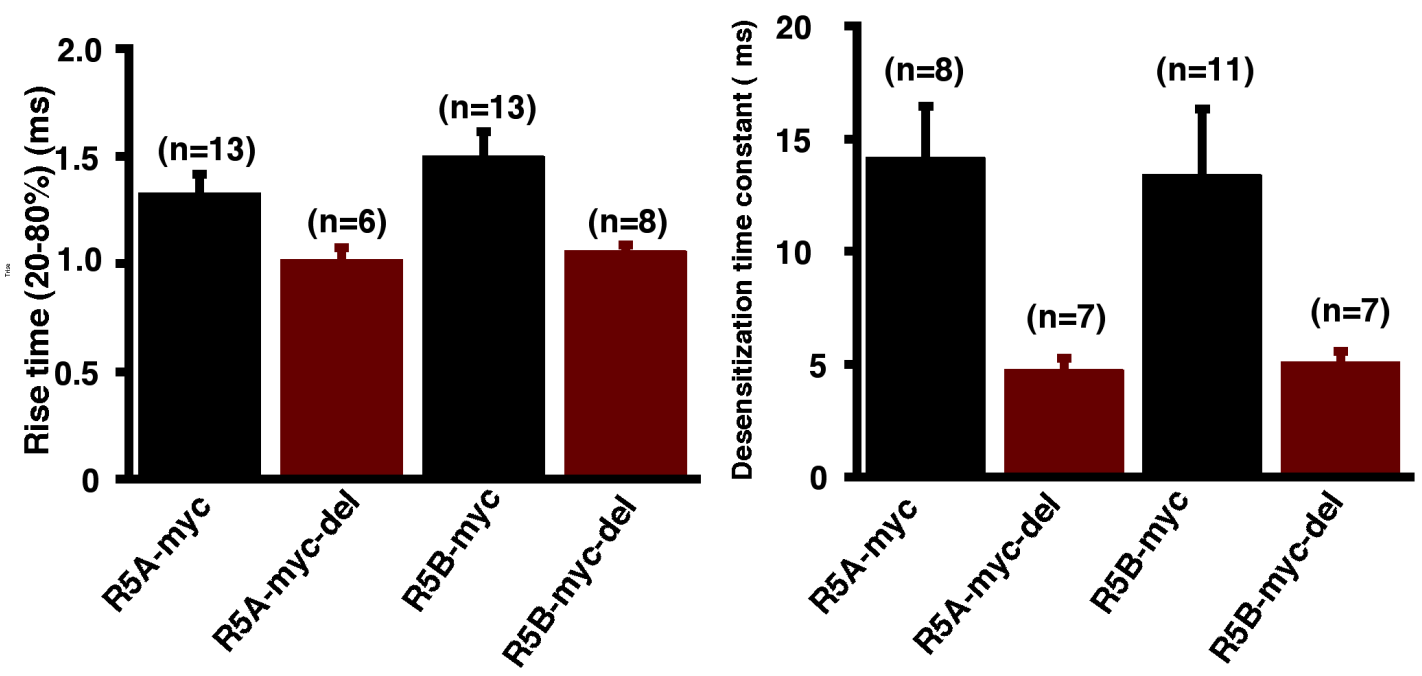

\section{Figure 3. Analysis of activation and desensitization phase of GluR5 isoforms}

(A) The bar plot describes the analysis of rise time (20-80\%) measured for whole cell responses in the presence of $10 \mathrm{mM}$ glutamate. The rise time of R5A and R5B isoforms were not significantly different. Furthermore, the deletion of 15 amino acids in these isoforms resulted in slightly but not significantly faster rise time (B) the bar plot describes the analysis of time constant of desensitization. The desensitizing phase of non-deleted or deleted isoforms was well fit by single exponential. $\mathrm{n}$ represents the number of whole cell recordings performed.

Based on these results, one can interpret that the fast desensitization in deletion mutants necessarily activates the receptors faster (see figure $3 \mathrm{~A}$ ) than their wild-type 
analogues (see figure 3B). This explains why mutant isoforms of R5 subunit have faster rise time as compared to their wild-type analogues.

\subsection{Effect on the amount of desensitization}

We measured the amount of desensitization (Peak/Steady state) from the same recordings for deletion mutants and wild-type isoforms of R5A and R5B subunits. We observed that the amount of desensitization is indeed affected by deleting the 15 amino acids either from R5A or R5B isoforms. The deletion mutant of R5A and R5B isoforms showed 1.8-1.9 fold larger peak/steady-state ratio as compared to their wildtype analogues (see Figure4).

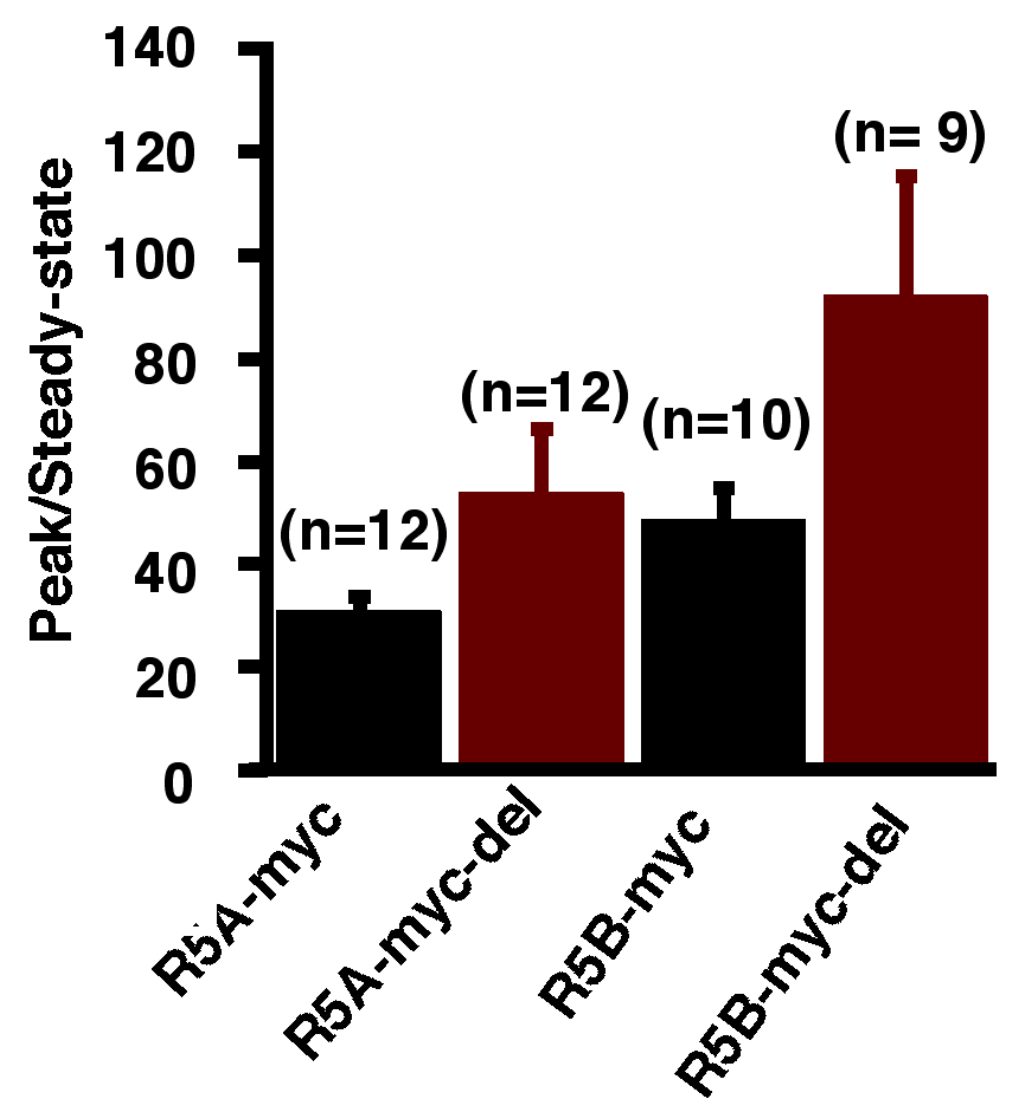

Figure 4. Analysis of the effect of N-terminal deletion in GluR5 isoforms on the amount of desensitization

The bar plot describes the analysis of the amount of desensitization (Peak/Steadystate) measured for whole cell responses of R5A, R5B and their N-teminal deletion mutants R5A-myc-del and R5B-myc-del in the presence of $10 \mathrm{mM}$ glutamate. $\mathrm{n}$ represents the number of whole cell recordings performed. 
As we did not observe any significant difference between R5A and GluR5B isoforms, the results suggest that both isoforms of R5 subunit may share very common properties of desensitization and other biophysical parameters. Furthermore, deletion of 15 amino acids from the $\mathrm{N}$-terminus region outside the S1-domain results in affecting the rate and amount of desensitization.

\section{DISCUSSION}

In this work, we have studied the gating and assembly mechanisms of AMPA and kainate receptors by using various molecular and electrophysiological approaches. Our major findings show that two subunits of AMPA receptor (R1 and R2) combines in preferential manner. The preferential assembly of these subunits reduces the variability of the functional phenotypes caused by assembly of two receptors having different functions.

We furthermore analysed pharmacological means of modifying the gating properties of AMPA receptors and found that the different modulators can affect the process of desensitization through different mechanisms. This is possibly by means of differential interaction of modulators with confirmationally different states of the receptor.

The analysis of biophysical properties of kainate receptor revealed the importance of two different domains. First, the expression of R6 subunit is dependent on the integrity of the C-terminus region. Secondly, the N-terminal splice variants of R5 subunit (R5A and B isoforms as compared to their deletion mutants) that affects the structure and function of ligand-binding domain led to a significant change in the degree of desensitization and hence may affect efficacy of receptor to transmit the synaptic signals.

\subsection{Mechanism of Heteromeric assembly of AMPA receptors and their functional implications}

In this study we have investigated the intersubunit interactions that direct AMPA receptor assembly, and influence channel gating. We found that the spatial arrangement of subunits determines the desensitization and rectification properties of the receptor/channel. The independent marker (assigned for desensitization and rectification) mutations used in this study led to surprisingly detailed insights into receptor structure and function. Key to these findings was the measurements from 
homomeric combination of receptors. The reproducibility and internal consistency of the data, and the predictive accuracy of the binomial assembly model were taken into account while assessing the validity of our analytical approach.

\subsubsection{Model assumptions}

The only assumption that was needed for the construction of a binomial model of homomeric R1 and homomeric R2 subunit assembly was that the channel phenotype switches from desensitizing to nondesensitizing (without any intermediate phenotypes exhibiting partial desensitization) depending on the combination of subunits. The absence of pronounced intermediate phenotype (see section 3.9.2, figure 10, and section 3.9.4, figure 12) in our experiments suggested that there is a clear switch between strong desensitization phenotype (>90\% say) and weak desensitization $(<10 \%)$. As for example, the data from $1 \mathrm{Q}+1 \mathrm{Q}(\mathrm{L}-\mathrm{Y})$ and $2 \mathrm{Q}+2 \mathrm{Q}(\mathrm{L}-\mathrm{Y})$ (Figure 16A) accurately follow the trajectory predicted by model $2 \mathrm{~A}$. If one of the subunit configurations possesses the intermediate phenotype characteristics then the data would have followed an intermediate trajectory between the trajectories predicted by various models. Further cross verifications are provided with $1 \mathrm{Q}+1 \mathrm{R}(\mathrm{L}-\mathrm{Y})$ and 2Q+2R(L-Y) data set (Figure 18A). At $-30 \mathrm{mV}$ both trajectories accurately followed the predictions of model 2A. Switching to $+30 \mathrm{mV}$ resulted in selective silencing of the channels with the rectifying phenotype and the desensitization results were well described by model 2B (Figure 18A). If subunit assemblies contained intermediate phenotypes, it is extremely unlikely that all six independent results could be accurately described by binomial model. The simplest explanation would be that the L-Y phenotype switches cleanly and allow the spatial configurations in both GluR1 and GluR2 homomeric receptors. The model for homomeric AMPA receptor assembly thus stands on two assumptions. First, the L-Y marker does not influence assembly or channel properties and secondly a clean consistent phenotype switch occurs in subunits incorporating this marker during assembly of AMPA receptors. These are strongly documented with the data. Assumptions pertaining to the relative conductance of different subunit assemblies are not required at this stage to explain desensitization-to-nondesensitization phenotype switch. Taken together, the homomeric data and model based on the L-Y marker lead to important conclusions about the relationship between the spatial arrangement of subunits and receptor desensitization. 
The assembly of homomeric receptors was well described by Model 2 . According to the assumptions of model 2, two subunits are necessary for the conversion of the resulting channel to the marker phenotype. The presence of marker for desensitization in homomeric assembly followed polar heterodimer arrangement of subunits (model 2A). The presence of marker for rectification of homomeric receptors in homomeric assembly followed symmetric heterodimeric arrangement of subunits (model 2B). Our data suggest that homomeric subunits assemble in a stochastic fashion regardless of whether they carry the glutamine $(\mathrm{Q})$ or arginine $(\mathrm{R})$ in the channel pore region. As for example, model $2 \mathrm{~A}$ accurately predicts the phenotype expression pattern for the nondesensitizing marker in both $2 \mathrm{Q}+2 \mathrm{R}(\mathrm{L}-\mathrm{Y})$ and $2 \mathrm{Q}(\mathrm{L}-\mathrm{Y})+2 \mathrm{R}$ (Figure $18 \mathrm{~A}, \mathrm{~B})$.

A clean consistent phenotype switch occurs even when Q-R marker is incorporated into individual subunits during assembly process. In contrast to desensitization-to-nondesensitization phenotype switch, this requires additional assumptions regarding the single channel conductance and open probability of various Q-R combinations to necessarily model the linearization data. These assumptions are directly or indirectly supported by the results of single-channel recordings and mean variance noise analysis (see section 3.7, figure 7 and section 3.8, figure 8). In addition, the excellent agreement between the predictions of binomial assembly model and data obtained with many different subunit combinations confirms that the singlechannel parameters are approximately correct. As for example, model 2A accurately predicts the nondesensitization phenotype data for $1 \mathrm{Q}+1 \mathrm{Q}(\mathrm{L}-\mathrm{Y}), 2 \mathrm{Q}+2 \mathrm{Q}(\mathrm{L}-\mathrm{Y})$ and $1 \mathrm{Q}+1 \mathrm{R}(\mathrm{L}-\mathrm{Y}), 2 \mathrm{Q}+2 \mathrm{R}(\mathrm{L}-\mathrm{Y})$ combinations at $-30 \mathrm{mV}$. The former data set assumes similar conductance values for all subunit assemblies, while the latter combinations involve assemblies that are assigned by different conductance values. Using such variable preassigned conductance values, the prediction of model $2 \mathrm{~B}$ accurately describes the linearization data for $1 Q+1 R$ and $2 Q+2 R$ combination (see figure $16 B$ ), and the nondesensitization data for $1 \mathrm{Q}+1 \mathrm{R}(\mathrm{L}-\mathrm{Y})$ and $2 \mathrm{Q}+2 \mathrm{R}(\mathrm{L}-\mathrm{Y})$ at $+30 \mathrm{mV}$ (see figure 18A). If there were a serious error with the conductance parameters for assemblies incorporating Q-R marker, then the internal consistent results would not be expected. Rather, the Q-R data set should have shown a marked deviation from the binomial predictions. Furthermore, the absence of pronounced intermediate phenotypes due to differential block of spermine $(50 \mu \mathrm{M})$ to different subunit configurations reinforced us to consider a clean switch in phenotype from "total 
block" to "slight block" by intracellular spermines.

\subsubsection{Theoretical, Biochemical and Structural consequences in the assembly of AMPA receptors}

From theoretical point of view, several aspects become clear. First, the random binomial assembly model accurately predicts all the homomeric data but is not adequately sufficient to describe the heteromeric data. Secondly, the alternative model that assumes selective assembly provides a clear description of the heteromeric data in all cases. Third, the only model that can account for all aspects of the heteromeric data is the one that assumes selective assembly of subunits with point-symmetric configuration.

From biochemical point of view, the quantitative description of linear relationship between plasmid concentration and protein expression imply that there is no intrinsic difference between the expression pattern of R1 and R2 subunits. These data alone do not explicitly allow one to conclude that both proteins are expressed in identical total concentrations. However, given the identical number of functional channels in the patch, such conclusion may be strengthened. In support to this, the excellent predictions of the binomial model and homomeric data for several different subunit combinations (by changing the markers between R1 and R2 subunit) showed an unchanged behavior in pattern of expression during AMPA receptor assembly.

From structural point of view, one can consider that in homomeric assembly, subunit interactions within the dimerized extracellular domain control the desensitization process. However a linear conductance requires two argininecontaining subunits positioned on opposite sides of the channel pore region. The information obtained from homomeric receptors were used to investigate the assembly of heteromeric receptors. The results on heteromeric receptor showed that the spatial arrangement of subunits determine the desensitization and rectification properties of the receptor/ structure and function.

The analysis of R1/R2 heteromers in specific showed that the distribution of subunit assemblies strongly favor a stoichiometry of two R1 and two R2 subunits in a symmetric arrangement. Furthermore, the prediction of dimer formation between R1 and R2 subunit explained well our data on the requirements for block of desensitization in coexpression experiments of nondesensitizing subunits with wildtype subunits. Regardless of which subunit is carrying the mutation, models 
which predicted that the wildtype and mutant receptors need to pair-up for the block of desensitization, fitted the data much better than random distribution of the nondesensitizing subunits. This is nicely complemented by structural data from (Armstrong and Gouaux, 2000). Furthemore, this data suggest that the manipulation leading to block of desensitization (by L-Y point mutation) is likely to take place at the interface between the two subunits in the dimer. Interestingly, not all manipulations are equally effective. For the L-Y cotransfection experiments, one of the two receptors need to carry the mutation. We take this difference as an indication that heteromeric dimers interact more intimate during gating and therefore may have different, perhaps higher likelihood of assembly.

\subsubsection{Possible mechanisms for AMPA receptor assembly}

One could explain the selective assembly of symmetric heterodimers by two hypothetical mechanisms. Symmetry-controlled assembly and association-controlled assembly may be two exemplary features of how cooperativity by subunit-subunit interaction allow the arrangment of subunits within a tetramer. In these structures, asymmetric subunit assemblies are destabilized either due to steric hindrance caused by various geometric factors or interface mismatch between subunits. Geometric factors may be intersubunit bond lengths (see section 3.10.10).

During selective subunit assembly if one assumes that subunit-subunit binding is reversible then the expressed subunits will be present in one of the three stable symmetric configurations. This would then lead to a binomial distribution similar to Model 4 (Figure 14). The second hypothesis regarding subunit assembly mechanism assumes that monomer consecutively associates to form a dimer. The dimer of a dimer will then lead to the formation of a full tetramer. This assumption is along with the idea that R2 dimerizes with R1 more rapidly than other subunit pairings. These mechanisms may be favorable if the physical profile of the right-hand interface of R2 subunit matches closely with the left-hand interface of R1 subunit (associationcontrolled assembly)

Based on these mechanisms, we tested these processes by constructing a chemical kinetic model of the assembly process. These models predicted that the best fit can be obtained for the phenotype expression data if R2 binds to R1 subunit five times faster than other subunit combinations. The rate of formation of dimers are likely due to the need for intersubunit interactions to protect functionally critical 
domains from either the membrane or aqueous environment. The other forms may be unstable or have short half lives of stability. This additionally strengthen the view regarding the assembly of symmetric heterodimers. At least in in-vitro system the actual mechanism of association of two subunits may be some combination of these two mechanisms, or it may involve interactions with other proteins. We would not impose that our model is the only model to explain the mechanistic basis of assembly process, as other schemes with different assumptions can also be made to fit the data set. However, the cooperativity among subunits seems to provide a simple explanation for the observed gating phenotype. Experimental evidences are furthermore necessary to know the precurssors and competents of dimeric subunit association.

Thus it remains clear that the assembly mechanism prefers to incorporate both R1 and R2 subunits where possible, thereby producing a relatively homogenous population of AMPA receptors. Furthermore, the assembly mechanism in which subunit recognition sites are created (as heteromerization progresses) may insure the fidelity of the assembly process and contribute for the quality control mechanisms.

\subsubsection{Functional significance of selective assembly in AMPA receptors}

Combining different subunits in a protein that functions as a multimer such as an ion channel is an attractive mechanism to allow diversity of function. Modular construction can produce multimers with diverse and flexible properties controlled by the gene expression levels. It is known from previous studies that the pattern and level of expression of receptor subunits is developmentally regulated. This further alters the kinetics, pharmacology, and other properties of the assembled receptor (Dingledine et al., 1999). Thus spatial arrangement of subunits within a receptor becomes an important factor for the optimal function of the channel. However, if positioning of the subunits in the multimer were important for proper function, assembly in a random fashion would yield only in fraction of properly functioning channels and a significant proportion of "malfunctioning" channels. The perhaps most important property in AMPA channels in this respect is the control of permeation by a mixture of arginine containing $\mathrm{R} 2$ receptors and the glutamine containing R1, 3 or 4 (Hume et al., 1991; Sommer et al., 1991). By examining the blocking properties of spermine in homomers, we found that R2 subunits like to be across from each other to efficiently convert the phenotype to linearly conducting channels without, perhaps, affecting 
single channel conductance (Swanson et al., 1997b). Recent data on dimerization in homomers, either by biochemical assembly assays for R4 (Kuusinen et al., 1999) or the crystallisation of extracellular ligand binding core of R2 subunits as a dimer (Armstrong and Gouaux, 2000) allows us to speculate that dimerization is a attractive mechanism to assure proper assembly in AMPA receptors. Although these data were obtained from homomers, we have shown in our studies that heteromers also form dimers. Heterodimerization furthermore strongly limit the variability of assemblies that can take place. As a result, only channels with a defined function are formed.

The ligand-binding domain of R2 subunit also has a dimer interface in the crystal structure with residues important for desensitization being positioned at the points of intersubunit contact (Armstrong et al., 1998). Thus dimerization may not only be a general principle guiding the assembly of multimeric channels but may also involve the residues important for gating processes. Our results support the suggestion that interactions between residues at the dimer interface of a tetramer influence the gating process. However, one cannot exclude the possibility that other subunit interactions (for example, at the transmembrane region or the $\mathrm{N}$-terminus preceding the S1-binding region) are important for preferential assembly of symmetric heterodimers (Ayalon and Stern-Bach, 2001).

It is likely that the spatial arrangement of the subunits is important for optimal function. Evolution may therefore have provided a mechanism that promotes the assembly of subunits in their optimal configuration. Functional evidence from other type of channels have also shown that dimerization is the key mechanism for subunitsubunit interaction. For example K-channels (Tu and Deutsch, 1999) and cyclic nucleotide-gated channels occur in dimer form (Liu et al., 1998).

In summary, the dimerization of subunits may occur through symmetrycontrolled or association controlled assembly processes. These mechanisms might have evolved to reduce structural and functional heterogeneity in the receptor population. The finding that $\mathrm{R} 1 / 2$ subunits assembles as a pair of dimers demonstrates a new mechanism of receptor behavior and will improve our understanding to know the role of these receptors in the central nervous system. Similar mechanisms are thought to control the assembly of other functional multimers. 


\subsection{Probing mechanism of AMPA receptor desensitization with chemically defined AMPA receptor modulators}

The main goal of second part of our work was to examine the mechanism of two exemplary AMPA receptor modulators of a functionally defined class, the ampakines CX546 and CX516 (benzoylpiperidine derivatives). We compared the action of benzoylpiperidine with structurally distinct class of modulators called benzothiadiazide. We used cyclothiazide and IDRA21 from this class to determine their mode of action. CX546 reduced the degree of desensitization on recombinant or native AMPA receptors more potently than CX516 or IDRA21. On recombinant receptors CX546 was not as effecient as cyclothiazide. We found that CX516/CX546 increased the absolute steady-state amplitudes of $\mathrm{R} 2_{\text {flip }}$ more than $\mathrm{R} 1_{\text {flip. }}$. which was significant. However, the relative amount of desensitization was affected approximately to the same extent in presence of CX546. In comparison to native receptors, the effect of CX546 on homomeric receptors was clearly less efficient. CX546 was as potent as cyclothiatzide in enhancing the the synaptic charge of the EPSC. In desensitization lacking AMPA-receptor mutants (R1L497Y) we observed specific increase of agonist affinity in the presence of CX546. Additionally CX546 slowed the current deactivation of nondesensitizing mutant in contrast to CX516 and IDRA21. Kinetic analysis showed that CX546 binds specifically to the agonist bound, nondesensitized receptor, and acts by destabilizing the desensitized conformation of the receptor.

The higher efficacy of CX546 on native receptors as compared to recombinant receptors may be due to unknown abundance of flip/flop splice variants in the native cells. Although the neurons used in this study are dominated by flip spliced variants (as judged by almost complete block of desensitization by cyclothiazide). (Fleck et al., 1996; Patneau et al., 1993) and although structural analogues of CX546 and CX516 seem not to strongly prefer flop spliced AMPA receptors (Arai et al., 2000), these drugs may act more efficient on flop containing, native receptors. Further studies are needed to clarify these issues. 


\subsubsection{Distinguishing action of CX546 over other AMPA modulators on AMPA receptors}

We observed that the modulation of AMPA receptor desensitization by CX546 was different from benzothiadizine derivatives. Both the kinetics of the modulator itself and the kinetics of its action was different from other modulators tested in this work. It contrasts the action of IDRA 21 or cyclothiazide (Yamada and Tang, 1993) by (1) reducing the AMPA receptor desensitization not by decreasing the rate of desensitization but by increasing the rate out of the desensitized state (2) its apparent specificity of binding to a kinetically defined receptor state(s). At molecular level, cyclothiazide blocks desensitization by its slow binding and unbinding kinetics (Partin et al., 1994) to a site formed by pocket at the interface of two subunits (Armstrong et al., 1998; Partin, 2001). Preincubation experiments showed that the binding of cyclothiazide to the receptor is independent of the receptor being bound by the agonist. Cyclothiazide has additional effects such as (1) weak inhibitory action (Patneau et al., 1993) and (2) decreasing the apparent affinity of the agonist for the receptor (Kessler et al., 1996; Stern-Bach et al., 1998). In contrast to the action of cyclothiazide, CX546 increases apparent agonist affinity by nearly threefold. Secondly, CX546 does not show any inhibitory effect. Based on their distinct mechanism of action one can postulate that although CX546 modulate desensitization as potently as benzothiadiazides, they may not share the same binding site for the course of action. Competitive binding studies are necessary to understand these issues in detail.

\subsubsection{Mechanism of the action of CX546}

Thermodynamically one could explain that the action of CX546 is to destabilize the desensitized state. The mechanism of action was determined by preincubation experiment. We observed that preequilibration of the receptor with CX546 is not sufficient to inhibit desensitization. However, the coapplication of CX546 with glutamate on AMPA receptor resulted in full efficacy of the modulator. These results suggested that the binding of CX546 to an agonist-unbound state was 
poor. Binding and unbinding kinetic experiments supported the evidences for the actual mode of action. When CX546 was removed, we observed a slower recovery from potentiation in presence of saturating agonist concentration as compared intermediate agonist concentrations. These results imply that unbinding of CX546 is promoted by unbinding of the agonist. The mechanism may be bidirectional since we observed a threefold slower current deactivation kinetics in nondesensitizing receptors. On contrary, if once the receptor enter into desensitized state, then the onset of the modulator action was observed to become extremely slow. However, we noticed that the onset was accelerated at intermediate agonist concentration implying a constant fraction of receptors entering into agonist bound state yet not being desensitized. This may imply that either the binding of CX546 to the desensitized state is strongly inhibited or destabilizing action of CX546 on the desensitized state is not effecient. This imply that CX546 may preferentially bind to the agonist bound, nondesensitized state of the receptor. Mechanistically, binding of the agonist might cause a conformational change that permits binding of CX546. Secondly, the binding of the modulator destabilizes the desensitized conformation and slows unbinding kinetics of the agonist.

\subsubsection{Physiological and pharmacological implications of the action of CX546 on AMPA receptors}

The differential mechanism of the action of CX546 (which is reflected by its binding kinetics) has potentially important therapeutic implications. The importance of this modulator lie in its specificity to bind to the agonist bound, closed (and/or open) state, but not to bind or act on to the desensitized or agonist unbound state. This mechanism of action may be important for finding their effectiveness during normal and excessive glutamate release. CX546 although enhances the agonist affinity for the receptor, it may be less effective in potentiating glutamate responses under ambient agonist concentration. Ambient concentration of agonist is expected to occur during build-up of glutamate in phases of cell death. Under these conditions, CX546 may be ineffective due to its lower efficiency in binding to the desensitized state of the receptor.

The side effects expected from CX546 are fewer as compared to benzothidiazides IDRA 21 (Impagnatiello et al., 1997; Uzunov et al., 1995; Zivkovic et al., 1995) and with other ampakines (Ingvar et al., 1997; Lynch et al., 1997; Staubli 
et al., 1994b) due to its specific binding properties for kinetic states of the receptor. The second important application may be its stronger removal of desensitization at R2 containing subunits. In this scenario, one may expect a preferential activation of calcium impermeable (receptors containing R2 subunits) AMPA receptors. This may reduce the chances of increasing the potentially toxic calcium-influx mediated directly by opening of AMPA channels. However further experiments are required to know the therapeutic potential of CX546 in memory and cognitive enhancements similar to other ampakines, which have been observed to facilitate LTP in vitro and in vivo (Staubli et al., 1994b).

\subsection{Biophysical properties and the role of kainate receptors in synaptic transmission}

In the last part of the thesis work, we studied the structural and functional aspects of some dominant isoforms of kainate receptors. Our focus was targeted to probe the functional relevance of splicing in the C-terminus in R5/R6 subunit as well as the N-terminus of GluR5 subunit. Similar to other subunits of AMPA receptors, kainate receptor of R6 subtype interacts with several postsynaptic proteins in neurons. Furthermore, this alters the intrinsic gating properties of the receptors. It was thus interesting to understand how the biophysical properties are altered by perturbing the structural elements in the C-terminus of GluR6 subunit. The mutants lacking 4,14, and 54 amino acids from the C-terminus tail of R6 subunits were constructed for this purpose.

A sub part of this work also includes the characterization of gating properties of R5 subunit. This subunit occurs in different splice variant isoforms that differs in both the length and residues within the $\mathrm{C}$ and $\mathrm{N}$-terminus region. We were motivated to know if these structural variations in $\mathrm{C}$ and $\mathrm{N}$-terminus have any important relevance in determining the gating kinetics of GluR5 subunit.

\subsubsection{Physiological role of C-terminus region of GluR6 subunit}

Recent observations have demonstrated that the members of the SAP90/PSD95 family colocalize and associate with kainate receptors in vivo and in vitro. Indeed, it has been shown that the coexpression of SAP90 with recombinant kainate receptor subunits decreases receptor desensitization (Garcia et al., 1998). The clustering of GluR6 channels is mediated by binding of the SAP90 PDZ1 domain to the four C- 
terminal amino acids of GluR6 subunit (ETMA). The deletion of C-terminal amino acids has been shown to abrogate the ability to bind and cluster to SAP 90 (Garcia et al., 1998). In the current study we have focused our understanding on the biophysical properties of C-terminal deletion mutants of GluR6 subunit. The long C-terminus of GluR6 subunit contain a number of hydrophobic residues which may serve as substrate for phosphorylation by several key enzymes like PKA, PKC and CamKII. The 54 amino acid cassette at the C-terminus region comprises of 3 serines and 2 threonine residues. The deletion of short peptides of 4 and 14 amino acids (R6-4 and R6-14) from R6 subunit lacked only threonine residues but the construct R6-54 (lacking 54 amino acid) lacked threonine along with two serine residues. Our data suggest that (1) the deletion of ETMA motif does not affect the biophysical properties of R6 subunit to a significant extent when exposed to saturating concentrations of glutamate (2) Removal of theronine residues (R6-4 and R6-14 deletion mutants) did not affect the gating kinetics of the R6 subunit in contrast to wild-type receptors. However, removal of serine residues (as observed by the deletion mutant R6-54) affected only the level of R6 subunit expression without affecting the gating kinetics.

These results are in contrast to other neurotransmitter systems like dopamine receptors. For example, a single substitution of threonine residue to alanine in the carboxy tail abolished agonist-induced phosphorylation and desensitization of the receptor (Lamey et al., 2002). The surprising effect of unaltered biophysical properties in the deletion mutants lacking several serine and threonine residues imply that the gating characteristics are controlled by different mechanisms that might not work mechanistically similar to other ligand gated channels. For instance, phosphorylation of nicotinic acetylcholine receptors by PKA increases the rate of fast desensitization (Huganir et al., 1986). Studies on phosphorylation of R6 C-terminus region are furthermore required for clarifying these issues. Our experimental approach involves a simple expression system, the HEK 293 system. The expression of individual subunits in HEK cells provides a simpler way to determine the intrinsic gating properties as compared to the complexities involved in the receptors being expressed by neuronal cells.

\subsubsection{Potential physiological functions of the extracellular and intracellular domains in GluR5 subunit}

As described in the earlier part of this chapter, the importance of GluR5 
subunit arises due to their expression as different $\mathrm{C}$ and $\mathrm{N}$-terminus splice variant isoforms. The importance of these variations and their physiological relevance has so far not understood clearly.

Our results on R5 isoforms revealed that there is no significant difference in gating properties (rise time, amount of desensitization, and desensitization time constant) between R5A and R5B isoforms (data not shown). However a significant difference was observed in the time constant of desensitization and amount of desensitization when 15-amino acid cassette was deleted from R5A isoforms. This implies that the slowing of desensitization in $\mathrm{R} 5 \mathrm{~A}$ isoform is due to the presence of extra 15 amino acid cassette or one could envision that the extra 15 amino acid cassette can act as desensitization controlling pocket.

\subsection{Future directions}

Our work related to the properties of heteromeric glutamate receptors provide valuable insights into number of unresolved questions ranging from the biophysical details of the mechanism of receptor gating to the signals mediating subtype-specific assembly of selected subtypes of glutamate receptors. Assembly of AMPA receptors in several parts of adult brain primarily involves heteromerization of R1 with R2, or R2 with R3 subunits. The heteromerization is a highly regulated process in neuronal cells which play important role ranging from determining the optimal stoichiometry of receptor population to specific cellular signaling associated with them. The necessity for the formation of specific heteromeric assembly has been less clearly understood. In this study we have provided the biophysical basis for the assembly process and mechanism of gating by using heterodimer model that incorporates R1 and R2 subunit. These subunits are highly expressed in $90 \%$ of the synapses in neurons.

Our findings provide new insights to probe the intersubunit interaction of R2 with other AMPA receptors such as R3 and R4. The methodology we employed in utilizing the markers that were targeted for structurally two different regions of the individual subunits (R1 and R2 subunit) can be employed for determining the subunit stiochiometry and assembly mechanisms with other subunit combinations of AMPA receptors. Furthermore these experiments will reveal whether heterodimerization is specific for only $\mathrm{R} 1 / 2$ combination or any other subunit combination undergoes similar subunit interaction and assembly process. 
Providing convincing answers to these questions can not only help us to understand the protein-protein interaction and conformational changes in real-time but also will provide valuable insights to understand the dynamic molecular events that underlie inter and intramolecular subunit interaction in vivo. Patch clamp technique in addition to several fluorescent-imaging techniques (Emptage, 2001) can be utilized for this purpose. The specific oligomerization between subunits can be best understood by fluorescence studies such as FRET. FRET, the fluorescence resonance energy transfer has attracted the attention of both ionotropic and metabotropic receptors in recent years. Individual receptors as homomers or combination of receptors as heteromers can be studied by overexpressing CFP, YFP, GFP (cyan, yellow and fluorescence proteins) tagged to them using this technique.

These questions are important to fully understand the role of these receptors in neurological abnormalities that occurs in several areas in brain. For example, excessive activation of NMDA, AMPA and kainate receptors is associated with large increase in intracellular calcium, which triggers a cascade of events that ultimately may lead to neuronal apoptosis. AMPA/kainate receptors are involved in several brain disorders like Huntington, Alzheimers, amyotrophic lateral sclerosis (ALS) and glutamate-mediated excitotoxicity. Down regulation of GluR2 mRNA in vulnerable neurons are found to be key factors for transient forebrain or global ischaemia (Gorter et al., 1997; Pellegrini-Giampietro et al., 1997). Defect in RNA editing are also thought to be one of the several factors that may be involved in neurodegeneration. As for example, the early-onset epilepsy and postnatal lethality is found in editing deficient GluR-B allele in mice (Brusa et al., 1995). Neuronal apoptosis at several regions of brain have been observed to be caused by the factors that are closely linked to glutamate receptors like, spontaneous semi-dominant mouse neurological mutation (Lurcher) in delta subunits and selective death of purkinje cells that can lead to ataxia (Zuo et al., 1997).

Our results on kainate receptors provide the necessary information on the important regions that determines the gating process. We have shown that, perturbing C-terminus of GluR6 subunit does not affect the gating process. However, the gating kinetics is significantly altered when the 15 -amino acid residues just outside the S1 domain pertained to N-terminus of GluR5 subunit are deleted. Utilizing these results one may further proceed to know whether these perturbations made either in N- or Cterminus has any effect in heteromerization with other kainate receptor subunits. 
Furthermore, it is interesting to know how these deletion mutants affect the synaptic properties when expressed in hippocampal neurons.

The understanding of the biophysical properties of glutamate receptors (AMPA and kainate) in several brain regions thus becomes a central issue to probe their appropriate stoichiometry and composition based on cellular and developmental changes. Answers to these questions may be helpful for targeting new drugs that may prevent glutamate receptor mediated cellular effects and neurodegeneration.

\subsection{Significance of our findings}

Our findings may contribute specifically towards the better understanding of pharmacology and neural transmission of AMPA/kainate receptors. The identification of preferential interaction of R1 and R2 subunit and their spatial arrangement are necessary for their optimal function at central synapses. There are several advantages of building such symmetric oligomeric proteins in nature. This allow proteins to interact with high specificity and directionality, since protein-protein interfaces ensures that the symmetric arrangement is favored.

Our findings on the pharmacological effect and mechanism of ampakine derivative CX546 on AMPA receptors provide a gateway to find (1) its overall effect in selective neuroprotection through glutamatergic transmission similar to CX516 (Bahr et al., 2002) (2) its potential effect on learning and memory related processes in comparison to other neurotoxic modulators. These results may prove to be of high therapeutic significance. The mechanism of action of CX546 also provide hints on (1) how structural variations in modulators can be beneficial to prove their effects from neurotoxic to neuroprotective behavior at physiological level (2) rational drug design having minimal side-effects.

The studies of unaltered kinetics of C-terminal deletion mutants in R6 subunit provide hints that the gating kinetics of this receptor is independent of the components of the cytoplasmic region. The $\mathrm{C}$-terminus region controls the expression level of the receptor on the surface of the membrane. Such regions have been implicated by several studies to either complex with PDZ domains of postsynaptic density proteins or for controlling other intracellular signaling mechanisms. The role of extra 15 amino acid cassette outside the $\mathrm{S} 1$ domain (N-terminus region) in $\mathrm{R} 5$ subunit now provided us the hint for its function in slowing the desensitization process. This may have important physiological relevance at glutamatergic and GABAergic synapses. 


\section{REFERENCES}

Arai, A., Kessler, M., Ambros-Ingerson, J., Quan, A., Yigiter, E., Rogers, G., and Lynch, G. (1996). Effects of a centrally active benzoylpyrrolidine drug on AMPA receptor kinetics. Neuroscience 75, 573-585.

Arai, A. C., Kessler, M., Rogers, G., and Lynch, G. (2000). Effects of the potent ampakine CX614 on hippocampal and recombinant AMPA receptors: interactions with cyclothiazide and GYKI 52466. Mol Pharmacol 58, 802-813. Armstrong, N., and Gouaux, E. (2000). Mechanisms for activation and antagonism of an AMPA-sensitive glutamate receptor: crystal structures of the GluR2 ligand binding core . Neuron 28, 165-181.

Armstrong, N., Sun, Y., Chen, G. Q., and Gouaux, E. (1998). Structure of a glutamate-receptor ligand-binding core in complex with kainate. Nature 395, 913-917.

Ayalon, G., and Stern-Bach, Y. (2001). Functional assembly of AMPA and kainate receptors is mediated by several discrete protein-protein interactions. Neuron 31, 103-113.

Bahn, S., Volk, B., and Wisden, W. (1994). Kainate receptor gene expression in the developing rat brain. J Neurosci 14, 5525-5547.

Bahr, B. A., Bendiske, J., Brown, Q. B., Munirathinam, S., Caba, E., Rudin, M., Urwyler, S., Sauter, A., and Rogers, G. (2002). Survival signaling and selective neuroprotection through glutamatergic transmission. Exp Neurol 174, 37-47.

Barbour, B., Keller, B. U., Llano, I., and Marty, A. (1994). Prolonged presence of glutamate during excitatory synaptic transmission to cerebellar Purkinje cells. Neuron 12, 1331-1343.

Bass, B. L., Nishikura, K., Keller, W., Seeburg, P. H., Emeson, R. B., O'Connell, M. A., Samuel, C. E., and Herbert, A. (1997). A standardized nomenclature for adenosine deaminases that act on RNA. Rna 3, 947-949.

Baumbarger, P., Muhlhauser, M., Yang, C. R., and Nisenbaum, E. S. (2001). LY392098, a novel AMPA receptor potentiator: electrophysiological studies in prefrontal cortical neurons. Neuropharmacology 40, 992-1002. 
Behe, P., Stern, P., Wyllie, D. J., Nassar, M., Schoepfer, R., and Colquhoun, D. (1995). Determination of NMDA NR1 subunit copy number in recombinant NMDA receptors. Proc R Soc Lond B Biol Sci 262, 205-213.

Bekkers, J. M., and Stevens, C. F. (1991). Excitatory and inhibitory autaptic currents in isolated hippocampal neurons maintained in cell culture. Proc Natl Acad Sci U S A 88, 7834-7838.

Bertolino, M., Baraldi, M., Parenti, C., Braghiroli, D., DiBella, M., Vicini, S., and Costa, E. (1993). Modulation of AMPA/kainate receptors by analogues of diazoxide and cyclothiazide in thin slices of rat hippocampus. Receptors Channels 1, 267-278.

Bettler, B., Boulter, J., Hermans-Borgmeyer, I., O'Shea-Greenfield, A., Deneris, E. S., Moll, C., Borgmeyer, U., Hollmann, M., and Heinemann, S. (1990). Cloning of a novel glutamate receptor subunit, GluR5: expression in the nervous system during development. Neuron 5, 583-595.

Bowie, D., and Mayer, M. L. (1995). Inward rectification of both AMPA and kainate subtype glutamate receptors generated by polyamine-mediated ion channel block. Neuron 15, 453-462.

Brusa, R., Zimmermann, F., Koh, D. S., Feldmeyer, D., Gass, P., Seeburg, P. H., and Sprengel, R. (1995). Early-onset epilepsy and postnatal lethality associated with an editing-deficient GluR-B allele in mice. Science 270, 1677-1680.

Burnashev, N. (1996). Calcium permeability of glutamate-gated channels in the central nervous system. Curr Opin Neurobiol 6, 311-317.

Burnashev, N., Monyer, H., Seeburg, P. H., and Sakmann, B. (1992). Divalent ion permeability of AMPA receptor channels is dominated by the edited form of a single subunit. Neuron 8, 189-198.

Chen, C. A., and Okayama, H. (1988). Calcium phosphate-mediated gene transfer: a highly efficient transfection system for stably transforming cells with plasmid DNA. Biotechniques 6, 632-638.

Chittajallu, R., Braithwaite, S. P., Clarke, V. R., and Henley, J. M. (1999). Kainate receptors: subunits, synaptic localization and function. Trends Pharmacol Sci 20, 26-35.

Clements, J. D., and Westbrook, G. L. (1991). Activation kinetics reveal the number of glutamate and glycine binding sites on the N-methyl-D-aspartate receptor. Neuron 7, 605-613. 
Colquhoun, D., Jonas, P., and Sakmann, B. (1992). Action of brief pulses of glutamate on AMPA/kainate receptors in patches from different neurones of rat hippocampal slices. J Physiol (Lond) 458, 261-287.

Diamond, J. S., and Jahr, C. E. (1995). Asynchronous release of synaptic vesicles determines the time course of the AMPA receptor-mediated EPSC. Neuron 15, 1097-1107.

Dingledine, R., Borges, K., Bowie, D., and Traynelis, S. F. (1999). The glutamate receptor ion channels. Pharmacol Rev 51, 7-61.

Donevan, S. D., and Rogawski, M. A. (1995). Intracellular polyamines mediate inward rectification of $\mathrm{Ca}(2+)$-permeable alpha-amino-3-hydroxy-5-methyl-4isoxazolepropionic acid receptors. Proc Natl Acad Sci U S A 92, 9298-9302.

Dudel, J., Franke, C., and Hatt, H. (1990). Rapid activation, desensitization, and resensitization of synaptic channels of crayfish muscle after glutamate pulses. Biophys J 57, 533-545.

Emptage, N. J. (2001). Fluorescent imaging in living systems. Curr Opin Pharmacol 1, 521-525.

Ferrer-Montiel, A. V., and Montal, M. (1996). Pentameric subunit stoichiometry of a neuronal glutamate receptor. Proc Natl Acad Sci U S A 93, 2741-2744.

Fleck, M. W., Bahring, R., Patneau, D. K., and Mayer, M. L. (1996). AMPA receptor heterogeneity in rat hippocampal neurons revealed by differential sensitivity to cyclothiazide. J Neurophysiol 75, 2322-2333.

Garcia, E. P., Mehta, S., Blair, L. A., Wells, D. G., Shang, J., Fukushima, T., Fallon, J. R., Garner, C. C., and Marshall, J. (1998). SAP90 binds and clusters kainate receptors causing incomplete desensitization. Neuron 21, 727-739.

Gates, M., Ogden, A., and Bleakman, D. (2001). Pharmacological effects of AMPA receptor potentiators LY392098 and LY404187 on rat neuronal AMPA receptors in vitro. Neuropharmacology 40, 984-991.

Geiger, J. R., Melcher, T., Koh, D. S., Sakmann, B., Seeburg, P. H., Jonas, P., and Monyer, H. (1995). Relative abundance of subunit mRNAs determines gating and $\mathrm{Ca} 2+$ permeability of AMPA receptors in principal neurons and interneurons in rat CNS. Neuron 15, 193-204.

Gorter, J. A., Petrozzino, J. J., Aronica, E. M., Rosenbaum, D. M., Opitz, T., Bennett, M. V., Connor, J. A., and Zukin, R. S. (1997). Global ischemia induces 
downregulation of Glur2 mRNA and increases AMPA receptor-mediated Ca2+ influx in hippocampal CA1 neurons of gerbil. J Neurosci 17, 6179-6188.

Gouliaev, A. H., and Senning, A. (1994). Piracetam and other structurally related nootropics. Brain Res Brain Res Rev 19, 180-222.

Granger, R., Deadwyler, S., Davis, M., Moskovitz, B., Kessler, M., Rogers, G., and Lynch, G. (1996). Facilitation of glutamate receptors reverses an ageassociated memory impairment in rats. Synapse 22, 332-337.

Gregor, P., O'Hara, B. F., Yang, X., and Uhl, G. R. (1993). Expression and novel subunit isoforms of glutamate receptor genes GluR5 and GluR6. Neuroreport 4, 1343-1346.

Hall, R. A., Hansen, A., Andersen, P. H., and Soderling, T. R. (1997). Surface expression of the AMPA receptor subunits GluR1, GluR2, and GluR4 in stably transfected baby hamster kidney cells. J Neurochem 68, 625-630.

Hausser, M. (2001). Synaptic function: dendritic democracy. Curr Biol 11, R1012.

Heckmann, M., Bufler, J., Franke, C., and Dudel, J. (1996). Kinetics of homomeric GluR6 glutamate receptor channels. Biophys J 71, 1743-1750.

Hestrin, S. (1993). Different glutamate receptor channels mediate fast excitatory synaptic currents in inhibitory and excitatory cortical neurons. Neuron 11, 10831091.

Higgs, M. H., and Lukasiewicz, P. D. (1999). Glutamate uptake limits synaptic excitation of retinal ganglion cells. J Neurosci 19, 3691-3700.

Hollmann, M., and Heinemann, S. (1994). Cloned glutamate receptors. Annu Rev Neurosci 17, 31-108.

Holst, B. D., Vanderklish, P. W., Krushel, L. A., Zhou, W., Langdon, R. B., McWhirter, J. R., Edelman, G. M., and Crossin, K. L. (1998). Allosteric modulation of AMPA-type glutamate receptors increases activity of the promoter for the neural cell adhesion molecule, N-CAM. Proc Natl Acad Sci U S A 95, 2597-2602.

Hosey, M. M. (1999). What molecular events underlie heterologous desensitization? Focus on "receptor phosphorylation does not mediate cross talk between muscarinic M(3) and bradykinin B(2) receptors". Am J Physiol 277, C856-858. 
Huganir, R. L., Delcour, A. H., Greengard, P., and Hess, G. P. (1986). Phosphorylation of the nicotinic acetylcholine receptor regulates its rate of desensitization. Nature 321, 774-776.

Hume, R. I., Dingledine, R., and Heinemann, S. F. (1991). Identification of a site in glutamate receptor subunits that controls calcium permeability. Science 253, 1028-1031.

Impagnatiello, F., Oberto, A., Longone, P., Costa, E., and Guidotti, A. (1997). 7Chloro-3-methyl-3,4-dihydro-2H-1,2,4-benzothiadiazine S,S-dioxide: a partial modulator of AMPA receptor desensitization devoid of neurotoxicity. Proc Natl Acad Sci U S A 94, 7053-7058.

Ingvar, M., Ambros-Ingerson, J., Davis, M., Granger, R., Kessler, M., Rogers, G. A., Schehr, R. S., and Lynch, G. (1997). Enhancement by an ampakine of memory encoding in humans. Exp Neurol 146, 553-559.

Isaacson, J. S., and Nicoll, R. A. (1991). Aniracetam reduces glutamate receptor desensitization and slows the decay of fast excitatory synaptic currents in the hippocampus. Proc Natl Acad Sci U S A 88, 10936-10940.

Jonas, P., Racca, C., Sakmann, B., Seeburg, P. H., and Monyer, H. (1994). Differences in $\mathrm{Ca} 2+$ permeability of AMPA-type glutamate receptor channels in neocortical neurons caused by differential GluR-B subunit expression. Neuron 12, 1281-1289.

Jonas, P., and Sakmann, B. (1992). Glutamate receptor channels in isolated patches from CA1 and CA3 pyramidal cells of rat hippocampal slices. J Physiol $455,143-171$.

Jonas P, and Monyer H. Ionotropic Glutamate Receptors in the CNS. Berlin: Springer, 1999

Jones, M. V., and Westbrook, G. L. (1996). The impact of receptor desensitization on fast synaptic transmission. Trends Neurosci 19, 96-101.

Kamboj, S. K., Swanson, G. T., and Cull-Candy, S. G. (1995). Intracellular spermine confers rectification on rat calcium-permeable AMPA and kainate receptors. J Physiol (Lond) 486, 297-303.

Kawamoto, S., Uchino, S., Xin, K. Q., Hattori, S., Hamajima, K., Fukushima, J., Mishina, M., and Okuda, K. (1997a). Arginine-481 mutation abolishes ligandbinding of the AMPA-selective glutamate receptor channel alpha1-subunit. Brain Res Mol Brain Res 47, 339-344. 
Kawamoto, S., Uchino, S., Xin, K. Q., Hattori, S., Hamajima, K., Fukushima, J., Mishina, M., and Okuda, K. (1997b). Arginine-481 mutation abolishes ligandbinding of the AMPA-selective glutamate receptor channel alpha1-subunit. Brain Res Mol Brain Res 47, 339-344.

Kessler, M., Arai, A., Quan, A., and Lynch, G. (1996). Effect of cyclothiazide on binding properties of AMPA-type glutamate receptors: lack of competition between cyclothiazide and GYKI 52466. Mol Pharmacol 49, 123-131.

Knox, B. E., Khorana, H. G., and Nasi, E. (1993). Light-induced currents in Xenopus oocytes expressing bovine rhodopsin. J Physiol 466, 157-172.

Koh, D. S., Burnashev, N., and Jonas, P. (1995). Block of native Ca(2+)permeable AMPA receptors in rat brain by intracellular polyamines generates double rectification [published erratum appears in J Physiol (Lond) 1995 Nov 1;488(Pt 3):843]. J Physiol (Lond) 486, 305-312.

Kohler, M., Burnashev, N., Sakmann, B., and Seeburg, P. H. (1993). Determinants of $\mathrm{Ca} 2+$ permeability in both TM1 and TM2 of high affinity kainate receptor channels: diversity by RNA editing. Neuron 10, 491-500.

Koike, M., Tsukada, S., Tsuzuki, K., Kijima, H., and Ozawa, S. (2000). Regulation of kinetic properties of GluR2 AMPA receptor channels by alternative splicing. J Neurosci 20, 2166-2174.

Kullmann, D. M. (2001). Presynaptic kainate receptors in the hippocampus: slowly emerging from obscurity. Neuron 32, 561-564.

Kupper, J., Ascher, P., and Neyton, J. (1996). Probing the pore region of recombinant $\mathrm{N}$-methyl-D-aspartate channels using external and internal magnesium block. Proc Natl Acad Sci U S A 93, 8648-8653.

Kuusinen, A., Abele, R., Madden, D. R., and Keinanen, K. (1999). Oligomerization and ligand-binding properties of the ectodomain of the alphaamino-3-hydroxy-5-methyl-4-isoxazole propionic acid receptor subunit GluRD. J Biol Chem 274, 28937-28943.

Lamey, M., Thompson, M., Varghese, G., Chi, H., Sawzdargo, M., George, S. R., and O'Dowd, B. F. (2002). Distinct residues in the carboxyl tail mediate agonist-induced desensitization and internalization of the human dopamine D1 receptor. J Biol Chem 277, 9415-9421. 
Lampinen, M., Pentikainen, O., Johnson, M. S., and Keinanen, K. (1998). AMPA receptors and bacterial periplasmic amino acid-binding proteins share the ionic mechanism of ligand recognition. Embo J 17, 4704-4711.

Larson, J., Lieu, T., Petchpradub, V., LeDuc, B., Ngo, H., Rogers, G. A., and Lynch, G. (1995). Facilitation of olfactory learning by a modulator of AMPA receptors. J Neurosci 15, 8023-8030.

Laube, B., Kuhse, J., and Betz, H. (1998). Evidence for a tetrameric structure of recombinant NMDA receptors. J Neurosci 18, 2954-2961.

Lauterborn, J. C., Lynch, G., Vanderklish, P., Arai, A., and Gall, C. M. (2000). Positive modulation of AMPA receptors increases neurotrophin expression by hippocampal and cortical neurons. J Neurosci 20, 8-21.

Lerma, J., Paternain, A. V., Rodriguez-Moreno, A., and Lopez-Garcia, J. C. (2001). Molecular physiology of kainate receptors. Physiol Rev 81, 971-998.

Leuschner, W. D., and Hoch, W. (1999). Subtype-specific assembly of alphaamino-3-hydroxy-5-methyl-4-isoxazole propionic acid receptor subunits is mediated by their n-terminal domains. J Biol Chem 274, 16907-16916.

Li, F., Owens, N., and Verdoorn, T. A. (1995). Functional effects of mutations in the putative agonist binding region of recombinant alpha-amino-3-hydroxy-5methyl-4-isoxazolepropionic acid receptors. Mol Pharmacol 47, 148-154.

Li, P., Kerchner, G. A., Sala, C., Wei, F., Huettner, J. E., Sheng, M., and Zhuo, M. (1999). AMPA receptor-PDZ interactions in facilitation of spinal sensory synapses. Nat Neurosci 2, 972-977.

Lindau, M., and Neher, E. (1988). Patch-clamp techniques for time-resolved capacitance measurements in single cells. Pflugers Arch 411, 137-146.

Liu, D. T., Tibbs, G. R., Paoletti, P., and Siegelbaum, S. A. (1998). Constraining ligand-binding site stoichiometry suggests that a cyclic nucleotide-gated channel is composed of two functional dimers. Neuron 21, 235-248.

Lomeli, H., Mosbacher, J., Melcher, T., Hoger, T., Geiger, J. R., Kuner, T., Monyer, H., Higuchi, M., Bach, A., and Seeburg, P. H. (1994). Control of kinetic properties of AMPA receptor channels by nuclear RNA editing. Science 266, 1709-1713.

Lynch, G. (1998). Memory and the Brain: Unexpected Chemistries and a New Pharmacology. Neurobiol Learn Mem 70, 82-100. 
Lynch, G., Granger, R., Ambros-Ingerson, J., Davis, C. M., Kessler, M., and Schehr, R. (1997). Evidence that a positive modulator of AMPA-type glutamate receptors improves delayed recall in aged humans. Exp Neurol 145, 89-92.

Mano, I., Lamed, Y., and Teichberg, V. I. (1996). A venus flytrap mechanism for activation and desensitization of alpha-amino-3-hydroxy-5-methyl-4-isoxazole propionic acid receptors. J Biol Chem 271, 15299-15302.

Mano, I., and Teichberg, V. I. (1998). A tetrameric subunit stoichiometry for a glutamate receptor-channel complex. Neuroreport 9, 327-331.

Mehta, S., Wu, H., Garner, C. C., and Marshall, J. (2001). Molecular mechanisms regulating the differential association of kainate receptor subunits with SAP90/PSD-95 and SAP97. J Biol Chem 276, 16092-16099.

Melcher, T., Maas, S., Herb, A., Sprengel, R., Seeburg, P. H., and Higuchi, M. (1996). A mammalian RNA editing enzyme. Nature 379, 460-464.

Mennerick, S., and Zorumski, C. F. (1994). Glial contributions to excitatory neurotransmission in cultured hippocampal cells. Nature 368, 59-62.

Monyer, H., Seeburg, P. H., and Wisden, W. (1991). Glutamate-operated channels: developmentally early and mature forms arise by alternative splicing. Neuron 6, 799-810.

Mosbacher, J., Schoepfer, R., Monyer, H., Burnashev, N., Seeburg, P. H., and Ruppersberg, J. P. (1994). A molecular determinant for submillisecond desensitization in glutamate receptors. Science 266, 1059-1062.

Nagarajan, N., Quast, C., Boxall, A. R., Shahid, M., and Rosenmund, C. (2001). Mechanism and impact of allosteric AMPA receptor modulation by the ampakine CX546. Neuropharmacology 41, 650-663.

Neher, E., and Sakaba, T. (2001). Estimating transmitter release rates from postsynaptic current fluctuations. J Neurosci 21, 9638-9654.

Neher, E., and Stevens, C. F. (1977). Conductance fluctuations and ionic pores in membranes. Annu Rev Biophys Bioeng 6, 345-381.

O'Hara, P. J., Sheppard, P. O., Thogersen, H., Venezia, D., Haldeman, B. A., McGrane, V., Houamed, K. M., Thomsen, C., Gilbert, T. L., and Mulvihill, E. R. (1993). The ligand-binding domain in metabotropic glutamate receptors is related to bacterial periplasmic binding proteins. Neuron 11, 41-52. 
Otis, T., Zhang, S., and Trussell, L. O. (1996). Direct measurement of AMPA receptor desensitization induced by glutamatergic synaptic transmission. J Neurosci 16, 7496-7504.

Paas, Y. (1998). The macro- and microarchitectures of the ligand-binding domain of glutamate receptors [see comments] [published erratum appears in Trends Neurosci 1998 Apr;21(4):175]. Trends Neurosci 21, 117-125.

Partin, K. M. (2001). Domain interactions regulating ampa receptor desensitization. J Neurosci 21, 1939-1948.

Partin, K. M., Bowie, D., and Mayer, M. L. (1995). Structural determinants of allosteric regulation in alternatively spliced AMPA receptors. Neuron 14, 833843.

Partin, K. M., Fleck, M. W., and Mayer, M. L. (1996). AMPA receptor flip/flop mutants affecting deactivation, desensitization, and modulation by cyclothiazide, aniracetam, and thiocyanate. J Neurosci 16, 6634-6647.

Partin, K. M., Patneau, D. K., and Mayer, M. L. (1994). Cyclothiazide differentially modulates desensitization of alpha-amino-3-hydroxy-5-methyl-4isoxazolepropionic acid receptor splice variants. Mol Pharmacol 46, 129-138.

Partin, K. M., Patneau, D. K., Winters, C. A., Mayer, M. L., and Buonanno, A. (1993). Selective modulation of desensitization at AMPA versus kainate receptors by cyclothiazide and concanavalin A. Neuron 11, 1069-1082.

Paternain, A. V., Herrera, M. T., Nieto, M. A., and Lerma, J. (2000). GluR5 and GluR6 kainate receptor subunits coexist in hippocampal neurons and coassemble to form functional receptors. J Neurosci 20, 196-205.

Patneau, D. K., Vyklicky, L., and Mayer, M. L. (1993). Hippocampal neurons exhibit cyclothiazide-sensitive rapidly desensitizing responses to kainate. J Neurosci 13, 3496-3509.

Pellegrini-Giampietro, D. E., Gorter, J. A., Bennett, M. V., and Zukin, R. S. (1997). The GluR2 (GluR-B) hypothesis: $\mathrm{Ca}(2+)$-permeable AMPA receptors in neurological disorders. Trends Neurosci 20, 464-470.

Piserchio, A., Pellegrini, M., Mehta, S., Blackman, S. M., Garcia, E. P., Marshall, J., and Mierke, D. F. (2002). The PDZ1 domain of SAP90. Characterization of structure and binding. J Biol Chem 277, 6967-6973. 
Premkumar, L. S., Qin, F., and Auerbach, A. (1997). Subconductance states of a mutant NMDA receptor channel kinetics, calcium, and voltage dependence. J Gen Physiol 109, 181-189.

Raman, I. M., and Trussell, L. O. (1995). The mechanism of alpha-amino-3hydroxy-5-methyl-4-isoxazolepropionate receptor desensitization after removal of glutamate. Biophys J 68, 137-146.

Robert, A., Irizarry, S. N., Hughes, T. E., and Howe, J. R. (2001). Subunit interactions and AMPA receptor desensitization. J Neurosci 21, 5574-5586.

Rosenmund, C., Stern-Bach, Y., and Stevens, C. F. (1998). The tetrameric structure of a glutamate receptor channel [see comments]. Science 280, 15961599.

Seeburg, P. H. (1996). The role of RNA editing in controlling glutamate receptor channel properties. J Neurochem 66, 1-5.

Seeburg, P. H., Higuchi, M., and Sprengel, R. (1998). RNA editing of brain glutamate receptor channels: mechanism and physiology. Brain Res Brain Res Rev 26, 217-229.

Sheng, M., and Pak, D. T. (2000). Ligand-gated ion channel interactions with cytoskeletal and signaling proteins. Annu Rev Physiol 62, 755-778.

Sirvio, J., Larson, J., Quach, C. N., Rogers, G. A., and Lynch, G. (1996). Effects of pharmacologically facilitating glutamatergic transmission in the trisynaptic intrahippocampal circuit. Neuroscience 74, 1025-1035.

Smith, T. C., Wang, L. Y., and Howe, J. R. (2000). Heterogeneous conductance levels of native AMPA receptors. J Neurosci 20, 2073-2085.

Sommer, B., Burnashev, N., Verdoorn, T. A., Keinanen, K., Sakmann, B., and Seeburg, P. H. (1992). A glutamate receptor channel with high affinity for domoate and kainate. Embo J 11, 1651-1656.

Sommer, B., Keinanen, K., Verdoorn, T. A., Wisden, W., Burnashev, N., Herb, A., Kohler, M., Takagi, T., Sakmann, B., and Seeburg, P. H. (1990). Flip and flop: a cell-specific functional switch in glutamate-operated channels of the CNS. Science 249, 1580-1585.

Sommer, B., Kohler, M., Sprengel, R., and Seeburg, P. H. (1991). RNA editing in brain controls a determinant of ion flow in glutamate-gated channels. Cell 67, 11-19. 
Sommer, B., and Seeburg, P. H. (1992). Glutamate receptor channels: novel properties and new clones. Trends Pharmacol Sci 13, 291-296.

Staubli, U., Perez, Y., Xu, F. B., Rogers, G., Ingvar, M., Stone-Elander, S., and Lynch, G. (1994a). Centrally active modulators of glutamate receptors facilitate the induction of long-term potentiation in vivo. Proc Natl Acad Sci U S A 91, 11158-11162.

Staubli, U., Perez, Y., Xu, F. B., Rogers, G., Ingvar, M., Stone-Elander, S., Lynch, G., Thompson, D. M., Guidotti, A., DiBella, M., and Costa, E. (1994b). Centrally active modulators of glutamate receptors facilitate the induction of long-term potentiation in vivo. Proc Natl Acad Sci U S A 91, 11158-11162.

Stern-Bach, Y., Bettler, B., Hartley, M., Sheppard, P. O., O'Hara, P. J., and Heinemann, S. F. (1994). Agonist selectivity of glutamate receptors is specified by two domains structurally related to bacterial amino acid-binding proteins. Neuron 13, 1345-1357.

Stern-Bach, Y., Russo, S., Neuman, M., and Rosenmund, C. (1998). A point mutation in the glutamate binding site blocks desensitization of AMPA receptors. Neuron 21, 907-918.

Swanson, G. T., Gereau, R. W. t., Green, T., and Heinemann, S. F. (1997a). Identification of amino acid residues that control functional behavior in GluR5 and GluR6 kainate receptors. Neuron 19, 913-926.

Swanson, G. T., Kamboj, S. K., and Cull-Candy, S. G. (1997b). Single-channel properties of recombinant AMPA receptors depend on RNA editing, splice variation, and subunit composition. J Neurosci 17, 58-69.

Takamori, S., Rhee, J. S., Rosenmund, C., and Jahn, R. (2000). Identification of a vesicular glutamate transporter that defines a glutamatergic phenotype in neurons [see comments]. Nature 407, 189-194.

Tang, C. M., Shi, Q. Y., Katchman, A., and Lynch, G. (1991). Modulation of the time course of fast EPSCs and glutamate channel kinetics by aniracetam. Science 254, 288-290.

Thalhammer, A., Morth, T., Strutz, N., and Hollmann, M. (1999). A desensitization-inhibiting mutation in the glutamate binding site of rat alphaamino-3-hydroxy-5-methyl-4-isoxazole propionic acid receptor subunits is dominant in heteromultimeric complexes. Neurosci Lett 277, 161-164. 
Thurmond, R. L., Creuzenet, C., Reeves, P. J., and Khorana, H. G. (1997). Structure and function in rhodopsin: peptide sequences in the cytoplasmic loops of rhodopsin are intimately involved in interaction with rhodopsin kinase. Proc Natl Acad Sci U S A 94, 1715-1720.

Tong, G., Shepherd, D., and Jahr, C. E. (1995). Synaptic desensitization of NMDA receptors by calcineurin. Science 267, 1510-1512.

Traynelis, S. F., Silver, R. A., and Cull-Candy, S. G. (1993). Estimated conductance of glutamate receptor channels activated during EPSCs at the cerebellar mossy fiber-granule cell synapse. Neuron 11, 279-289.

Tretter, V., Ehya, N., Fuchs, K., and Sieghart, W. (1997). Stoichiometry and assembly of a recombinant GABAA receptor subtype. J Neurosci 17, 2728-2737. Trussell, L. O., and Fischbach, G. D. (1989). Glutamate receptor desensitization and its role in synaptic transmission. Neuron 3, 209-218.

Trussell, L. O., Thio, L. L., Zorumski, C. F., and Fischbach, G. D. (1988). Rapid desensitization of glutamate receptors in vertebrate central neurons. Proc Natl Acad Sci U S A 85, 4562-4566.

Trussell, L. O., Zhang, S., and Raman, I. M. (1993). Desensitization of AMPA receptors upon multiquantal neurotransmitter release. Neuron 10, 1185-1196.

Tu, L., and Deutsch, C. (1999). Evidence for dimerization of dimers in K+ channel assembly. Biophys J 76, 2004-2017.

Uzunov, D. P., Zivkovich, I., Pirkle, W. H., Costa, E., and Guidotti, A. (1995). Enantiomeric resolution with a new chiral stationary phase of 7-chloro-3-methyl3,4-dihydro-2H-1,2,4-benzothiadiazine S,S-dioxide, a cognition-enhancing benzothiadiazine derivative. J Pharm Sci 84, 937-942.

Valenta, D. C., Downing, J. E., and Role, L. W. (1993). Peptide modulation of ACh receptor desensitization controls neurotransmitter release from chicken sympathetic neurons. J Neurophysiol 69, 928-942.

Verdoorn, T. A., Burnashev, N., Monyer, H., Seeburg, P. H., and Sakmann, B. (1991). Structural determinants of ion flow through recombinant glutamate receptor channels. Science 252, 1715-1718.

Washburn, M. S., Numberger, M., Zhang, S., and Dingledine, R. (1997). Differential dependence on GluR2 expression of three characteristic features of AMPA receptors. J Neurosci 17, 9393-9406. 
Wisden, W., and Seeburg, P. H. (1993). A complex mosaic of high-affinity kainate receptors in rat brain. J Neurosci 13, 3582-3598.

Wo, Z. G., and Oswald, R. E. (1995). A topological analysis of goldfish kainate receptors predicts three transmembrane segments. J Biol Chem 270, 2000-2009. Yamada, K. A. (1998). Modulating excitatory synaptic neurotransmission: potential treatment for neurological disease? Neurobiol Dis 5, 67-80.

Yamada, K. A., and Rothman, S. M. (1992). Diazoxide blocks glutamate desensitization and prolongs excitatory postsynaptic currents in rat hippocampal neurons. J Physiol 458, 409-423.

Yamada, K. A., and Tang, C. M. (1993). Benzothiadiazides inhibit rapid glutamate receptor desensitization and enhance glutamatergic synaptic currents. J Neurosci 13, 3904-3915.

Zhang, D., Sucher, N. J., and Lipton, S. A. (1995). Co-expression of AMPA/kainate receptor-operated channels with high and low $\mathrm{Ca} 2+$ permeability in single rat retinal ganglion cells. Neuroscience 67, 177-188.

Zivkovic, I., Thompson, D. M., Bertolino, M., Uzunov, D., DiBella, M., Costa, E., and Guidotti, A. (1995). 7-Chloro-3-methyl-3-4-dihydro-2H-1,2,4 benzothiadiazine S,S-dioxide (IDRA 21): a benzothiadiazine derivative that enhances cognition by attenuating DL-alpha-amino-2,3-dihydro-5-methyl-3-oxo4-isoxazolepropanoic acid (AMPA) receptor desensitization. J Pharmacol Exp Ther 272, 300-309.

Zuo, J., De Jager, P. L., Takahashi, K. A., Jiang, W., Linden, D. J., and Heintz, N. (1997). Neurodegeneration in Lurcher mice caused by mutation in delta2 glutamate receptor gene. Nature 388, 769-773. 


\section{ACKNOWLEDGEMENTS}

I would first like to thank Prof. Dr. Erwin Neher who provided me the fellowship to carry out my Ph.D. in his department. Prof. Neher's kindful advices at several points helped me to have better perspective in my scientific thinking. I would like to thank my supervisor Dr. Christian Rosenmund who taught me patch clamp technique and constantly inspired me in all ways during my thesis work. I thank him for creating a good encouraging environment during my work and stay in his laboratory. His critical advices and guidance at each step made me to realize the importance of bridging the collective information to experimental reality. With the constant support of Prof. Neher and Dr. Rosenmund this thesis work was successfully completed.

I would like to thank Dr. Michael Mansour who taught me patch clamp technique and collaborated in this work. He was involved immensely in the project designing and modeling work presented in AMPA receptor assembly. His critical advices were very important for me to improve my skills in electrophysiolgy. I am highly thankful for his constant support through out my Ph.D. work as well as during the preparation of the manuscript. I thank him for critically revising and shaping this manuscript.

I wish to thank Ina Herfort for her excellent technical assistance. I wish to thank Dr. Ralph Nehring who performed the biochemical assays described in the work of AMPA receptor assembly. I wish to thank Dr. John.D. Clements who also was involved in modeling work of AMPA receptor assembly. I would like to thank Dr. Yael Stern-Bach who provided us AMPA receptor constructs. I wish to thank Christoph Mulle and Françoise coussen who collaborated on the kainate receptor project.

I wish to thank Dr. Ralph Schneggenburger for his friendly nature and scientific discussions with clarity at several instances. I thank him for critically 
revising the thesis work. I thank Alexander Mayer for critically revising the manuscript.

I wish to thank my friend Albrecht Sigler and Christoph Quest who helped me in several aspects during my Ph.D. time. I wish to thank all my lab colleagues and people in the department for their scientific stimulation and professional support throughout my Ph.D. thesis work.

This thesis is dedicated to my father, mother, sister and late grandmother for their constant source of encouragement and inspiration through out my life. 


\section{LIST OF PUBLICATIONS}

1. Mansour M, Nagarajan N, Nehring RB, Clements JD, Rosenmund C (2001) Heteromeric AMPA Receptors Assemble with a Preferred Subunit Stoichiometry and Spatial Arrangement. Neuron, 32, 841-853.

2. Nagarajan N, Quast C, Boxall AR, Shahid M, Rosenmund C (2001) Mechanism and Impact of AMPA receptor modulation by ampakine CX546. Neuropharmacology, 41, 650-663. 


\section{CURRICULUM VITAE}

21. 05.1974

$1979-1986$

$1986-1989$

1989-1991

$1991-1994$

October 1994

$1995-1997$

1997

1997-1999

$1999-2002$

$17-9-2002$
Born in Mysore, Karnataka, India, as elder son of Nagarajan Subbarao and Mysore Subbarao Pushpavalli.

Primary schooling at Royal English School, Mysore, Karnataka, India.

Secondary schooling at Marimallappas High School, Mysore, Karnataka, India.

Pre University course at Marimallappas Junior college, Mysore, Karnataka, India.

Bachelors of Science in Physics, Chemistry and Mathematics from Mysore University, Karnataka, India.

Obtained the 'Bachelor of Science'

Masters of Science from Vikram University, Ujjain, Madhya Pradesh, Indien.

Obtained "Master of Science" in Industrial Chemistry. Master thesis was in "Lipase catalysed esterification of palmitic and stearic acid with lactic acid" under supervision of Dr. S. Divakar from Central Food technological research Institute, Mysore, Karnataka, India

Research fellow at Department of Chemistry, Indian Institute of Technology, Bombay, India.

Ph.D. student at Max-Planck-Institute for Biophysical Chemistry Gottingen in the group of PD Dr. Christian Rosenmund, Department of Membrane Biophysics (Prof. Dr. Erwin Neher).

Naveen Nagarajan 